\title{
New insights in the electrical treatment of atrial fibrillation
}

Citation for published version (APA):

Timmermans, C. C. M. M. (2000). New insights in the electrical treatment of atrial fibrillation. [Doctoral Thesis, Maastricht University]. Datawyse / Universitaire Pers Maastricht. https://doi.org/10.26481/dis.20000128ct

Document status and date:

Published: 01/01/2000

DOI:

$10.26481 /$ dis.20000128ct

Document Version:

Publisher's PDF, also known as Version of record

\section{Please check the document version of this publication:}

- A submitted manuscript is the version of the article upon submission and before peer-review. There can be important differences between the submitted version and the official published version of record.

People interested in the research are advised to contact the author for the final version of the publication, or visit the DOI to the publisher's website.

- The final author version and the galley proof are versions of the publication after peer review.

- The final published version features the final layout of the paper including the volume, issue and page numbers.

Link to publication

\footnotetext{
General rights rights.

- You may freely distribute the URL identifying the publication in the public portal. please follow below link for the End User Agreement:

www.umlib.nl/taverne-license

Take down policy

If you believe that this document breaches copyright please contact us at:

repository@maastrichtuniversity.nl

providing details and we will investigate your claim.
}

Copyright and moral rights for the publications made accessible in the public portal are retained by the authors and/or other copyright owners and it is a condition of accessing publications that users recognise and abide by the legal requirements associated with these

- Users may download and print one copy of any publication from the public portal for the purpose of private study or research.

- You may not further distribute the material or use it for any profit-making activity or commercial gain

If the publication is distributed under the terms of Article $25 \mathrm{fa}$ of the Dutch Copyright Act, indicated by the "Taverne" license above, 
New insights in the electrical

treatment of atrial fibrillation 
(9) Carl Timmermans, Maastrich 2000

ISBN 9052782709

vormgeving en druk: Datawyse / Universitaire Pers Maastricht 


\title{
New insights in the electrical treatment of atrial fibrillation
}

\author{
Proefschrift
}

ter verkrijging van de graad van doctor aan de Universiteit Maastricht, op gezag van de Rector Magnificus, Prof. dr. A.C. Nieuwenhuijzen Kruseman, volgens het besluit van het College van Decanen, in het openbaar te verdedigen

op vrijdag 28 januari 2000 om 14.00 uur

door

Carl Timmermans

geboren te Hasselt, België

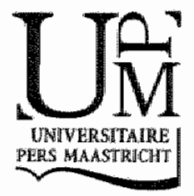




\section{Promotor:}

Prof. dr. H.J.J. Wellens

Co-promotor:

Dr. L.M. Rodriguez

Beoordelingscommissie:

Prof. dr. R.S. Reneman (voorzirter)

Prof. dr. M.A. Allessie

Prof. dr. H.J.G.M. Crijns (Rijksuniversiteit Groningen)

Prof. dr. S. Lévy (Centre Universitaire de Marseille, France)

Prof. dr. H.A.J. Struijker Boudier 
aan mijn ouders 



\section{Contents}

9 CHAPTER 1

Introduction

$15 \quad$ CHAPTER 2

Effect of electrode length on atrial defibrillation thresholds.

$25 \quad$ CHAPTER 3

Decreasing the number of leads required for an implantable atrial defibrillator: use of a new 2-lead system.

35 CHAPTER 4

Effect of butorphanol tartrate on shock related discomfort during internal. arrial defibrillation.

$49 \quad$ CHAPTER 5

Immediate reinitiation of atrial fibrillation following internal atrial defibrillation.

$61 \quad$ CHAPTER 6

Atrioverter: an implantable device for the treatment of atrial fibrillation.

$77 \quad$ CHAPTER 7

Spontaneous episodes of atrial fibrillation after implantation of the Metrix ${ }^{\text {Th }}$ Atrioverter: observations on treated and nontreated episodes.

91 CHAPTER B

Are electrophysiological changes induced by longer lasting atrial fibrillation reversible? Observations using the atrial defibrillator.

99 CHAPTER 9

Management of atrial arrhythmias secondary to severe congenital heart disease with the Atrioverter.

105 CHAPTER 10

Use of sedation during cardioversion with the implantable atrial defibrillator. 
113 CHAPTER II

Initial clinical experience with ambulatory use of an implantable atrial defibrillator for conversion of atrial fibrillation.

131 SUMMARY

137 SAMENVATTING

143 DANKWOORD

145 CURRICULUM VITAE

147 PUBLICATIONS 


\section{Introduction}

Since external direct current electrical cardioversion of arrial fibrillation was introduced by Lown et al in 1962', little has changed in the electrical treatment of this arrhythmia. External cardioversion is a safe and effective merhod of terminating atrial fibrillation. Nevertheless, the procedure requires general anesthesia and has limited applicability in patients with frequent atrial fibrillation episodes.

Significant progress was made when the exrernal defibrillation paddles or skin parches were gradually replaced by defibrillation electrodes. An esophageal electrode - cutaneous patch configuration allowed the cardioversion of patients with atrial fibrillation with an amount of energy three to four times lower than the energy required for conventional external cardioversion ${ }^{2}$. An early attempt to obtain lower atrial defibrillation thresholds using a single right atrial electrode in combination with a cutaneous parch was disappointing ${ }^{3}$. However, a randomized comparison of this technique with conventional external cardioversion demonstrated a higher immediate success rate of internal cardioversion compared to external cardiover$\operatorname{sion}^{4}$. In the first study where both defibrillation electrodes were located in the right heart, none of the patients with atrial fibrillation were successfully cardioverted ${ }^{5}$. Only after experimental studies in sheep, it was recognized that the optimal lead configuration for internal defibrillation of atrial fibrillation required electrodes encircling as much of the fibrillating atrium as possible ${ }^{6}$. In that study, four right atrial electrode positions (superior vena cava, right atrial appendage, mid and low right atrium) and three left atrial positions (coronary sinus, left pulmonary artery, left axillary subcutaneous parch) were evaluated. Additionally, the efficacy of monophasic and biphasic waveforms was compared. It was shown that a $3 / 3 \mathrm{msec}$ biphasic shock delivered between the right atrium and the coronary sinus had the lowest defibrillation threshold $(1.3 \pm 0.4 \mathrm{~J}$ )). The remarkably low amounts of energy to convert atrial fibrillation in animals prompted several centers to investigate the use of biatrial shocks in patients with atrial fibrillation ${ }^{7.9}$. In those studies, carhe ter-based atrial defibrillation was possible with energies ranging from 1 to $8 \mathrm{~J}$. These favorable preliminary results, together with the obvious success of the implantable cardioverter defibrillator for the treatment of ventricular arrhythmias prompted the development of a similar device designed to recognize and terminate atrial fibrillation. Thereafter, further research was mainly focussed on the following, initially defined, primary requirements for the acceptance of an implantable atrial defibrillator: safety, efficacy and patient tolerability of shock delivery 10 .

The safery of internal atrial defibrillation is crucial especially since atrial fibrillation is a not life threatening arrhythmia. Although $R$ wave-synchronized external 
atrial defibrillation has been safely performed in humans, experimental animal studies demonstrated that not all $\mathbb{R}$ waves are appropriate for timing a synchronized internal atrial defibrillation shock. Factors such as shocks delivered on $\mathrm{R}$ waves with a short preceding ventricular cycle length ( $<300 \mathrm{msec})$ and shocks delivered after a long-short sequence increased the risk of ventricular proarrhythmia ${ }^{11,12}$. Other circumstances in which some parts of the ventricular myocardium recovered later than orhers, like for example in bundle branch block, increased the risk of induction of ventricular fibrillation when an atrial defibrillation shock was synchronized to a QRS complex with a preceding R-R interval of $<380 \mathrm{msec}^{13}$. In clinical practice, using the right atrium to coronary sinus vector for atrial defibrillation, no ventricular arrhythmias are induced when appropriate $\mathrm{R}$-wave synchronization is performed and shocks are delivered only after $R-R$ intervals above $500 \mathrm{msec}^{14-16}$. Depending on the localization of the defibrillation electrode in relation to the sinus node and the AV node, internal atrial defibrillarion may result in transient bradycardia requiring ventricular pacing 6 .

A prospective, multicenter study confirmed the efficacy and safety of biphasic shocks delivered berween a right atrium and coronary sinus electrode in 14.1 patients with different subsets of atrial fibrillation ${ }^{15}$. Paroxysmal $(\leq 7$ days) atrial fibrillation was successfully terminated in $46(92 \%)$ of 50 parients, chronic ( $>30$ days) arrial fibrillation in $37(70 \%)$ of 53 patients, intermediate ( $>7$ days, $\leq 30$ days) atrial fibrillation in $16(89 \%)$ of 18 patients and induced atrial fibrillation in $16(80 \%)$ of 20 patients. The mean conversion threshold was $1.8 \pm 1.3 \mathrm{~J}$ in the induced group, $2.0 \pm$ $0.97 \mathrm{~J}$ in the paroxysmal group, $2.8 \pm 1.0 \mathrm{~J}$ in the intermediate group and $3.6 \pm 1.4 \mathrm{~J}$ in the chronic group. The conversion energy was significantly higher in the chronic group than in the other groups of atrial fibrillation and increased significantly with the duration of the arrhythmia and with left atrial size. No ventricular arrhythmia was induced. Most of the unsuccessful cardioversions were probably due to termination of the procedure if atrial defibrillation could not be achieved with a $10 \mathrm{~J}$ shock. A comparison of all published data is nor only difficult because of the variable use of a pre-established energy limit for termination of the procedure ${ }^{14-17}$, but also because of the hererogenous populations treared ${ }^{14,17,18}$, the evaluation of several technological improvements of the defibrillation system ${ }^{19-21}$, and the differences in lead configurations ${ }^{22}$. In Chapter 2 , the effect of electrode length on atrial defibrillation thresholds is discussed. It was found that the use of longer electrodes did not lower the defibrillation requirements for restoration of sinus rhythm. In Chapter 3 the reduction of the number of leads required for an implantable atrial defibrillator was evaluated. The examined 2-lead system appeared to be inferior to the conventional 3-lead system for implementation in an implantable atrial defibrillator.

Tolerability of internal low energy shocks is the third issue that will determine the acceptance of an implantable atrial defibrillator because atrial defibrillation shocks are intended to be delivered to a conscious patient in a non-emergent situation. Initial reports which measured the response of patients to atrial shocks 
suggested that shocks $>0.5$ to $1 \mathrm{~J}$ would not be tolerated without heavy sedation or analgesia ${ }^{14,23}$. Subsequent experience suggested that these conclusions were erroneous, because the investigators did not place the delivery of a shock in their proper context. Most of these patients did not suffer from symptomatic episodes of atrial fibrillation. Furthermore, shocks were delivered without adequate parient education, and the aversion to a shock was not compared to the aversion to the alternative: remaining in atrial fibrillation and/or seeking therapy using conventional methods. Shock-related discomfort may be due to pain and/or fear and may result from several factors such as skeletal muscle contraction, nerve stimulation, and psychological components ${ }^{14,16}$. Most studies attempted to cause less pain by lowering the atrial defibrillation threshold by use of improvements of the defibrillation system 19.21, shock characteristics $20,21,24$, and optimal electrode localization ${ }^{25,26}$ or by the administration of antiarrhythmic drugs ${ }^{27}$. In Chapter 4, the effect of intranasal butorphanol tartrate on shock related discomfort during internal atrial defibrillation was evaluated. This opioid analgesic decreased or stabilized the value of several pain variables and did not affect fear. The favorable effect of butorphanol on shock discomfort and ease in intranasal administration makes the drug suitable for use with an implantable atrial defibrillator.

Experimental studies in animals with induced atrial fibrillation have shown that electrophysiological changes in the atrium favored maintenance of atrial fibrillar $\operatorname{tion}^{28}$. These changes consisted in a shortening of the atrial effective refractory period and loss of the relation between the atrial effective refractory period duration and heart rate after prolonged periods of atrial fibrillation (electrical remodeling). Those experiments suggested that the findings on atrial electrophysiology after a long period of arrial fibrillation was the result of the arrhythmia. They argued in favor of prompt termination of atrial fibrillation to reverse the electrophysiologic changes favoring recurrences and the development of long-term atrial fibrillation. An important question in discussing the value of an implantable arrial defibrillator, is how the device will influence the natural history of atrial fibrillation. Electrical remodeling develops quickly and may account for an increased atrial vulnerability for recurrences of atrial fibrillation after cardioversion, also for those occurring immediately after defibrillation. In Chapter 5 , the immediate reinitiation of atrial fibrillation following internal atrial defibrillation was assessed. In that study, we found that immediate reinitiation of atrial fibrillation was always initiared by an atrial premature beat having a short coupling interval and was corrected by repeated shock delivery with or without preceding administration of pharmacologic agents.

In Chapters 6-11 the first experience with an implantable atrial defibrillator is described. In a prospective worldwide multicenter study, the safery and efficacy of the implanrable atrial defibrillator was evaluated in 51 patients without structural heart disease (Chapter 6 ). The effect of the device on the clinical course of the arrhythmia in these patients (Chapter 7) and the effect on atrial electrophysiology after longer lasting atrial fibrillation (Chapter 8 ) are discussed. Because atrial fibrilla- 
tion mainly occurs in patients with structural heart disease, the feasibility of the implantable atrial defibrillator was also evaluated in a patient with congenital heart disease (Chapter 9). The last 2 chapters address the use of sedation during cardioversion with the implantable atrial defibrillator as a measure of therapy tolerability (Chapter 10) and the ambulatory use of the device (Chapter 11).

\section{REFERENCES}

1. Lown $B$, Amarasingham $R$, Neuman I. New method for terminating cardiac arrhythmias: use of synchronized capacitor discharge. JAMA 1962:182:548-555.

2. MoNally EM, Mcyer EC, Langendorf R. Elective countershock in unanesthetized partents with use of an esophageal clectrode. Circulattion 1966;33:124-127.

3. Jain $\mathrm{SC}$, Bhatnagar VM, Atami RU, Awasthey P. Elective countershock in atrial fibrillation with an intracardiac elecrode: A preliminary report. J Assoc Physicians India 1970;18:821-824.

4. Lévy $S$, Lauribe P, Dolla E, Kou W, Kadish A, Calkins H, Pagannelli F, Moyal C, Bremondy M, Schork A, Shyr Y, Das S, Shea M, Gupta N, Morady F. A randomized comparison of external and internal cardioversion of chronic atrial fibrillation. Circulation 1992;86:1415-1420.

5. Narhan AW, Bexton $\mathrm{RS}_{*}$ Spurrell RAJ, Camm AJ. Internal transvenous low energy cardiowersion for the treatment of cardiac arrhythmias. Br Heart ] 1984:52:377-384.

6. Cooper RAS, Alfemess CA, Smith WA, Ideker RE. Internal cardioversion of atrial fibrillation in sheep. Circularion 1993;87:1673-1686.

7. Keane D, Sulke N, Cooke R, Jackson G, Sowton E. Endocardial cardioversion of arrial flutrer and fibrillation. Pacing Clin Electrophysiol 1993;16:928. Abstracr.

8. Johnson EE, Yarger MD, Wharton JM. Monophasic and biphasic waveforms for low energy internal cardioversion of atrial fibrillation in humans. Circulation 1993;88:I-592. Abstract..

9. Alt E, Schmitr C, Ammer R, Coenen M, Fotuhi P, Karch M, Blasini R. Initial experience with intracardiac atria』 defibrillation in patients with chronic atrial fibrillation. Pacing Clin Electrophysiol 1994:17 (Pt 11):1067-1078.

10. Léry $\$$, Camm J. An implantable arrial debbrillaror. An impossible dream? Circulation $1993 ; 87: 1769-1772$.

11. Ayers GM, Alferness CA, Ilina M, Wagner DO. Sirokman WA, Adams IM, Griffin JC. Ventricular proarhythmic effects of ventricular cycle length and shock strength in a sheep model of transwenous atrial defibrillation. Circulation 1994:89:413-422.

12. Kumagai K. Niwano S. Ayers GM, Waldo AL. Effects of the preceding R.R interval on the safery of atrial defibrillation via transwenous catherer electrodes in the sterile pericarditis model. J Am Coll Cardiol 1995;25:15A.

13. Keelan EET, Krum D, Hare J, Mughal K, Li H, Akhtar M, Jazayeri MR. Safery of atrial defibrillation shocks synchronized to narrow and wide QRS complexes during atrial pacing protocols simulating arrial fibrillation in dogs. Circulation 1997;96:2022-2030. 
14. Murgatroyd FD, Slade AKB, Sopher SM, Rowland E, Ward DE, Camm AJ. Efhocy and tolerability of transwenous low energy cardioversion of paroxysmal atrual fibrillation in humans.) Am Coll Cardiol 1995:25:1347-1353.

15. Lévy S, Ricard P, Lau CP, Lok NS, Camm AJ, Murgarroyd FD, Jordaens LJ, Kappenberger LJ, Brugada P, Ripley KL. Multicenter low energy tanswenous atrial defibrillation (XAD) rial results in different subsets of atrial fibrillation. I Am Coll Cardiol 1997;29:750-755.

16. Léry 5, Ricard P, Greunoun M, Yapo F, Trigano J, Mansouri C, Paganelli F. Low-energy cardioversion of spontaneous atrial fibrillation: immediate and long-ferm results. Circulation 1997;96:253-259.

17. Sancini M, Pandozi C. Toscano S, Castro A, Altamura G, Jeri AP, Gentuluci G, Villani M, Scianaro MC. Low energy intracardiac cardioversion of persistent atrial fibriliarion. Pacing Clin Electrophysiol 1998;21:2641-2650.

18. Schmirt C, Alt E, Plewan A, Ammer R, Leibig M, Karch M, Schömig A. Low energy intracardiac cardioversion after failed conventional extemal cardioversion of atriall fibrillation. I Am Coll Cardiol $1996 ; 28: 994-999$.

19. Tomassoni $G$, Newby KH, Kearney MM, Brandon M], Barold $H$, Natale A. Testing different biphasic waveforms and capacitances: effect on atrial defibrillation threshold and pain perception. I Am Coll Cardiol 1996;28:695-699.

20. Cooper RAS, Johnson EE, Wharton JM. Internal atrial defibrillation in humans: improved efficacy of biphasic waveforms and the importance of phase duration. Circulation 1997,95:1487-1496.

21. Cooper RAS, Plumb VJ, Epstein AE, Kay GN, Ideker RE. Marked reduction in internal arrial defibrillarion thresholds with dual-current pathways and sequential shocks in humans. Circulation $1998 ; 97: 2527-2535$

22. Alt E, Anmer R, Lehmann G, Schamitt C, Pasquantonio J Schömig A. Efficacy of a new balloon catherer for internal cardioversion of chronic atrial fibrillation without anaesthesia. Heart $1998 ; 79: 128-132$.

23. Steinhaus DM, Cardinal D, Mongeon L, Martson L, Waters M, Foley L, Corrigan S. Atrial defibrillation: are low-energy shocks acceptable to patients? Pacing Clin Electrophysiol 1996; 19:625. Abstract.

24. Ammer R, Alt E, Ayers $G_{1}$ Schmirt C, Pasquantonio J, Schmidt M, Pürer K, Schömig A. Pain threshold for low energy intracardiac cardioversion of arrial fibrillation with low or no sedarion. Pacing Clin Electrophysiol 1997;20:230-236.

25. Lok NS, Lau CP. Tse HF, Ayers GM. Clinical shock rolerability and effect of different righton antial elecrode locations on efficacy of low energy human transwenous arrial defibrillation using an implanrable lead syscem. J Am Coll Cardiol 1997;30:1324-1330.

26. Alt E, Schmirt C. Ammer $\mathbb{R}$, Plewan A, Evans F, Pasquantonio J, Ideker "I, Lehmann G, Pütter K. Schömig A. Effect of electrode position on outcome of low-energy intracardiac cardioversion of arrial fibrillation. Am J Cardiol 1997;79:621-625.

27. Lau CP, Lok NS. A comparison of transwenous atrial defibriliation of acute and chronic atrial fibrillarion and the effecr of intravenous sotalol on human atrial defibrillarion threshold. Pacing Clin Electrophysiol 1997;20:2442-2452.

28. Wijfiels MCEF, Kirchhof CJH), Dorland R. Allessie MA. Atrial fibrillation begets atrial fibrillarion. Circulation 1995;92:1954-1968. 

CHAPER 2

\section{Effect of electrode length on atrial defibrillation thresholds}

Carl Timmermans, MD; Luz-Maria Rodriguez, MD; Gregory M Ayers", MD;

Hendrik Lambert", PhD; Joep Smeets, MD; Hein JI Wellens, MD

From the Department of Cardiology, Academic Hospital Maastricht, Maastricht,

The Netherlands; and (") InnControl Inc., Redmond, Washington, USA

Abstract presented at the $48^{\text {th }}$ Ammual Scientific Session of the American College of Cardialogy 1998 ( Am Coll Cardiol 1998;31, 37A)

Published in / Cardiovasc Electroptystiol 1998:9:582-587 


\section{A B S T R A C T}

Introduction. Catheter-based electrodes have been used previously to terminate episodes of atrial fibrillation in animals and man. Typically, these electrodes span 6 to $7 \mathrm{~cm}$, and lowest energy requirements are achieved when these electrodes are positioned in the distal coronary sinus and in the right atrium. The purpose of this study was to evaluate the use of longer electrode lengths for atrial defibrillation.

Methods and Results. In 15 patients, two decapolar catheters were inserted, one into the distal coronary simus and one in the right atrium. To provide longer electrodes lengths, a third catheter was inserted and alternated positioned in the right atrium or coronary sinus. A 6-cm electrode span was obtained by using the distal 8 rings on the coronary sinus catherer or 8 consecutive electrodes on the right atrial catheter and. increased from 6 to $11 \mathrm{~cm}$ by connecting 5 consecutive, nonoverlapping rings of the third catheter with the 10 rings of the initial right atrial or coronary sinus catheter. Atrial defibrillarion thresholds were determined twice, in a randomized order, in each patient for each of the three combinations of electrode lengths. All 15 patients could be successfully converted to sinus thythm without complications; however, one patient could be converted reproducibly with only 2 of the 3 electrode combinarions. Mean thresholds were $306 \pm 102 \mathrm{~V}, 5.9 \pm 4.0 \mathrm{~J}$ for the $6 \mathrm{~cm} / 6 \mathrm{~cm}$ electrode length combination with an impedance of $72 \pm 18 \Omega$. For the electrode combination using the $11 \mathrm{~cm}$ electrode in the right atrium, the defibrillation threshold was $296 \pm$ $107 \mathrm{~V}, 5.8 \pm 3.9 \mathrm{~J}$ with an impedance of $61 \pm 17 \Omega$ and was $294 \pm 91 \mathrm{~V}, 5.6 \pm 3.6 \mathrm{~J}$ with an impedance of 55 t $11 \Omega$ for the $11-\mathrm{cm}$ electrode in the coronary sinus. There were no significant differences in defibrillation woltage or energy $(\mathrm{P}>0.05)$ associated with the longer electrode lengths; however, the longer electrode lengths did significanty lower shock impedance $(P<0.05)$.

Condusion. The use of longer electrodes, when using the right atrium to coronary sinus shock vector, does not lower the defibrillation requirements for restoration of sinus rhythm. 
Recently, internal cardioversion of atrial fibrillation using percutaneous trinswenous catheter electrodes has been successfully performed in man. $1-3,5,7,10-13$ This new method of cardioversion has several potential clinical applications, including: (1) the development of an implantable atrial defibrillator for the treatment of patients with recurrent symptomatic atrial fibrillation resistant to antiarrhythmic drugs; (2) elective endocardial cardioversion of inadvertent induced atrial fibrillation during an electrophysiologic study; (3) the treatment of post-cardiac surgery atrial fibrillation; and (4) treatment of patients resistant to external cardioversion. The amount of energy needed for internal cardioversion of atrial fibrillation is reduced using a right atrium to coronary sinus electrode configuration in combination with a biphasic shock waveform. ${ }^{4,5}$

To date, most studies performed on internal, carheter-based atrial defibrillation have used electrodes that have a linear span along the catheter of between 6 and 7 $\mathrm{cm}$. A systematic study of shorter electrode lengths revealed that shorter electrodes, i.e, $3 \mathrm{~cm}$, increased defibrillation thresholds in animals ${ }^{6}$ and in man. ${ }^{7}$ What remains unknown is whether electrodes $>6 \mathrm{~cm}$ would lower the atrial defibrillation requirements, as was seen for ventricular defibrillation. ${ }^{8}$ It was the purpose of this study to compare the atrial defibrillarion requirements for longer $(11 \mathrm{~cm})$ electrodes versus shorter $(6 \mathrm{~cm})$ electrodes in man, with the hypothesis that longer electrodes would lower atrial defibrillation thresholds.

\section{METHODS}

\section{STUDY POPULATION}

Fifteen consecurive parients with atrial fibrillation (12 men and 3 women; mean age $60 \pm 14$ years, range 33 to 86 ) in whom conversion to sinus rhythm was dinically indicated $(\mathrm{N}=14)$ or in whom atrial fibrillation was induced $(\mathrm{N}=1)$ underwent internal atrial defibrillation. Evaluation of the patients included a clinical history, physical examination, routine laboratory and thyroid function tests, a 12-lead ECG, and transthoracic or transesophageal echocardiography.

Patients were excluded from the study if they had any one of the following criteria: (1) an implanted artificial heart valve, (2) a previous history of a sustained ventricular arrhythmia, cardiac arrest, or congenital long QT syndrome, (3) atrial fibrillation due to reversible causes (e.g., hyperthyroidism), (4) a history of myocardial infarction or a revascularisation procedure within the past 6 months, (5) a history of thromboembolic events, (6) a left atrial thrombus diagnosed by transesophageal echocardiography obtained the day before cardiowersion, (7) digitalis toxicity, (8) significant electrolyte imbalance, (9) not having been anticoagulated for at least 4 weeks, or (10) an implanted pacing device. 
The administration of antiarrhythmic drugs was not interrupted for the procedure. Anticoagulation was interrupted 2 days before the procedure and restarted after the procedure. To perform a safe venous puncture, an INR value of 2.5 was required. The study protocol was approved by the Medical Ethics Committee of the Academic Hospital Maastricht, and informed consent was obtained from all patients.

\section{INTERNAL ATRIAL DEFIBRILLATION PROTOCOL}

Two temporary 6-French catheters (Elecath, Electro-Catheter Corp., Rahway, NJ, USA) consisting of 10 parallel stainless steel rings were inserted into the right femoral vein and positioned in the right atrium and distal coronary sinus. To provide longer electrode lengths, a third decapolar catheter was inserted and positioned, alternating its position in the right atrium or coronary sinus, in such a manner that the electrodes on the two catheters were aligned in a nonoverlapping manner (Fig. 1). A 6-cm electrode span, with an active surface area of $2.48 \mathrm{~cm}^{2}$, was created by using 8 consecutive rings on the right atrium or the distal 8 electrodes in the coronary sinus catheter. When the third catheter was positioned in either the coronary sinus or right atrium, the electrode span was increased from 6 to $11 \mathrm{~cm}$ by connecting 5 consecurive, nonoverlapping rings of this catheter with all 10 rings of the right atrium or coronary sinus catheter. The $11-\mathrm{cm}$ electrode span had an active surface area of $4.72 \mathrm{~cm}^{2}$. The multiple poles (either 8 or 15) of the catheter(s), lacated either in the right atrium or in the coronary sinus, were connected together using a custom-build "many pin to one pin" connector" to generate a single right atrial and a single coronary sinus electrode. These two functional electrodes, the right atrial as cathode and the coronary sinus as anode, were then attached to the outputs of an external defibrillator (Ventritex HVS-02, Sunnyvale, CA, USA). Biphasic defibrillarion shocks $(6 / 6 \mathrm{~ms})$, synchronized to the $\mathrm{R}$ wave, were delivered. To prevent inadvertent induction of ventricular fibrillation, shocks were delivered only after RR intervals $>500 \mathrm{~ms} .{ }^{9}$ In 1 parient, atrial fibrillation was induced by right atrial burst pacing. Atrial defibrillation thresholds were determined in a randomized order in each patient for each of the three combinations of electrodes and were determined twice for each electrode combination using a $0.5-\mathrm{J}$ start, a $0.5-\mathrm{J}$ step-up protocol until $3 \mathrm{~J}$, followed by $1.0-\mathrm{J}$ steps until cardioversion of atrial fibrillation occurred. During the procedure, conscious sedation was provided using intravenous midazolam $(0.1 \mathrm{mg} / \mathrm{kg})$. Oxygen saturation was monitored using a pulse oximeter, and the blood pressure was measured every 15 minutes. During the study, the 12-lead ECG and endocardial electrograms were recorded and stored by PC-EMS 3.0 (Cardiovascular Research Institute, Maastricht, The Netherlands). 


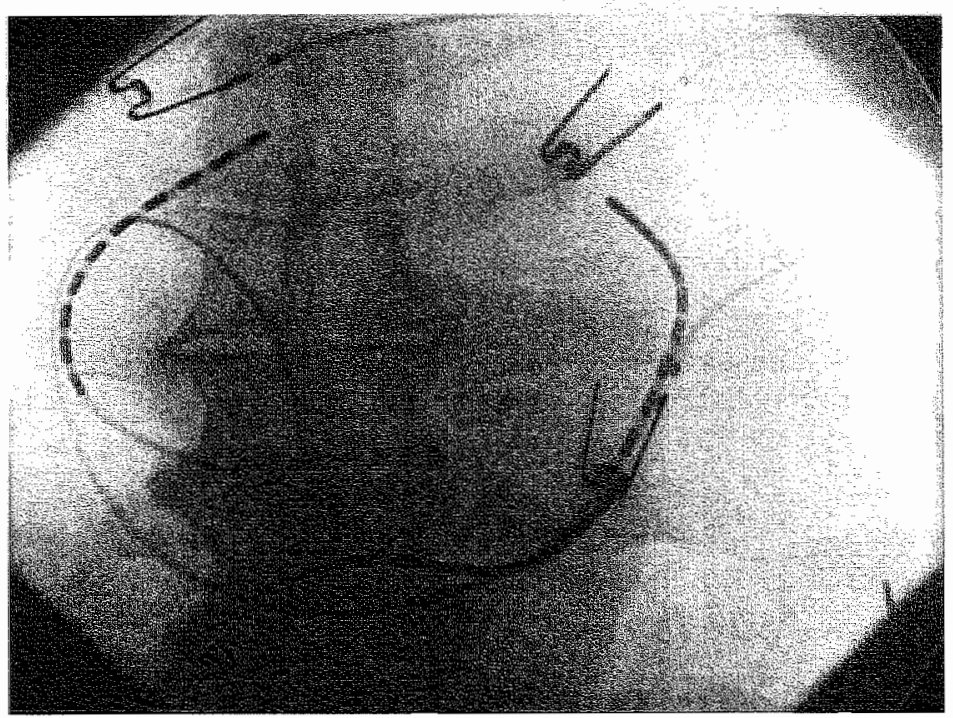

Figure 1. - Anteroposterior fluoroscopic projection of the posinion of the defibrillation catheters. Defibrillation was performed using a 6 -cm electrode span (bi electrodes) of the right atrial defibrillation catheter and a $11-\mathrm{cm}$ electrode span (15 consecutive electrodes) of the two coronary sinus defibrillation catheters.

\section{STATISTICAL ANALYSIS}

Continuous variables are expressed as mean values \pm SD and compared between groups using the Student's $t$ test. P $<0.05$ was considered statistically significant. Statistical power calculations were made using a clinically relevant difference if statistical significance was not achieved.

\section{RESULTS}

\section{CLINICAL CHARACTERISTICS}

The clinical and echocardiographic characteristics of the patients are reported in Table 1. As can be seen, the parients had a long history of atrial fibrillation with associated changes in left atrial diameter. Overall, the patients studied had clinical characteristics seen in the general population with atrial fibrillation, namely, there was a mixture of heart disease with four patients having lone atrial fibrillation and one parient having significant congenital heart disease (corrected transposition of great vessels). Four of the patients had failed one or more attempts at external cardioversion. Five patients had been previously treared with sotalol and six were treated with sotalol at the time of testing. Additionally, two patients had been treated previously 
with amiodarone, and six were treated with amiodarone at the time of testing. The antiarrhythmic agents that the patients were receiving at the time of internal defibrillation are shown in Table 1 .

Table 1. - Clinical and cehocardiographic characteristics of patienrs.

\begin{tabular}{|c|c|}
\hline Men/women & 1213 \\
\hline \multicolumn{2}{|l|}{ Age (years) } \\
\hline Mesun $\pm S D$ & $60 \pm 14$ \\
\hline Range & $33-86$ \\
\hline \multicolumn{2}{|l|}{ Duration AF as disease (years) } \\
\hline Meari $\approx$ \$D & $4.8 \pm 5.3$ \\
\hline Range & $0.33-15$ \\
\hline \multicolumn{2}{|l|}{ Duration treated AF cpisode (days) } \\
\hline Mcan \pm SD & $143 \pm 116^{*}$ \\
\hline Range & $3 \cdot 420$ \\
\hline \multicolumn{2}{|l|}{ Seructural heart disease } \\
\hline Ischemic & 3 \\
\hline Valvular & 2 \\
\hline Cardiomyopartly & 3 \\
\hline Hyperrensive & 2 \\
\hline Congenital & 1 \\
\hline Lone AF & 4 \\
\hline \multicolumn{2}{|l|}{ Cutrent antiarrhyrhmic drug. } \\
\hline Class I & 0 \\
\hline Class II & 1 \\
\hline Class III & 9 \\
\hline Class IV & 1 \\
\hline Digilalis & 4 \\
\hline Combination of antiarlyythic drugs & 3 \\
\hline No antiarrluythinic drug & 1 \\
\hline \multicolumn{2}{|l|}{ LA dianeter ( $\mathrm{mm}$ ) } \\
\hline Man is $\mathrm{SD}$ & $53 \pm 10$ \\
\hline Range & $42-80$ \\
\hline \multicolumn{2}{|l|}{ LVEF $(\%)$} \\
\hline $\begin{array}{l}\text { Mean i SD } \\
\text { Range }\end{array}$ & $\begin{array}{l}54 \pm 12 \\
35-66\end{array}$ \\
\hline
\end{tabular}

Datapresented are number of parients unless otherwise specified "calculated for 14 parients; AF: atrial fibrillation; LA: left atral; LVEF: lefte ventricular cjection fraction. 
Table 2. - Mean thresholds (range) for the diferent ellectrode length combinations tested.

\begin{tabular}{rllll}
\hline RA-CS length & Hestholds & Voltage $(N)$ & Energy $(0)$ & Impedance $(\Omega)$ \\
\hline $6 \mathrm{~cm}-6 \mathrm{~cm}$ & 30 & $306 \pm 102(160-520)$ & $5.9 \pm 40(1.4-15.6)$ & $72 \pm 18(47-128)$ \\
$11 \mathrm{~cm}-6 \mathrm{~cm}$ & 29 & $296 \pm 107(130-460)$ & $5.8 \pm 3.9(1.0-12.5)$ & $61 \pm 17(27-110)$ \\
$6 \mathrm{~cm}-11 \mathrm{~cm}$ & 30 & $294 \pm 91(160-510)$ & $5.6 \pm 3.6(1.5-15.5)$ & $55 \pm 11(37-83)$ \\
\hline
\end{tabular}

\#umber of shresholds measured; $\mathrm{CS}$ : coronary sinus; RA: righn arrium.

\section{OUTCOME OF INTERNAL ATRIAL DEFIBRILLATION AND COMPARISON OF ELECTRODE LENGTHS}

All patients were successfully cardioverred with all three of the electrode length combinations; however, one patient could not be reproducibly defibrillated with the $11-\mathrm{cm}$ right atrial and $6-\mathrm{cm}$ coronary sinus electrode length combination. This patient was later converted with a different configuration of electrode lengths. The highest threshold in any patient for any of the three length combination was $520 \mathrm{~V}$, $15.6 \mathrm{~J}$ (for the $6-\mathrm{cm} / 6-\mathrm{cm}$ combination). Despite successful defibrillation, four patients had recurrence of atrial fibrillation within minutes after one or more of the shocks. In three patients, additional shock delivery resulted in stable sinus rhythm. One patient who exhibited a pause-dependent recurrence of atrial fibrillation had the recurrence prevented using biatrial pacing (from the distal coronary sinus and right atrial electrode pair) in the postshock period. All patients were in stable sinus rhythm at the end of the procedure.

The results of the comparison of the atrial defibrillation thresholds for the three electrode length combinations is shown in Table 2. Comparing voltage, there was no significant difference $(\mathrm{P}>0.05)$ in the threshold voltage, and the two combinations with the longer electrode length in either the right atrium or coronary sinus had a threshold that was lower by 10 and $12 \mathrm{~V}$, respectively. The statistical power to detect a $20-V$ difference was $96.5 \%$ and was $99.99 \%$ to detect a $>30-V$ difference should one have existed. Despite the significant difference in impedance (discussed later), there was no significant difference in energy threshold $(P>0.05)$. Again, there was only a small difference present for the two combinations that used a longer electrode in eirher the right atrium $(0.1 \mathrm{~J}$ lower) or the coronary sinus $(0.3 \mathrm{~J}$ lower $)$. As with the voltage threshold data, there was adequate statistical power to detect a clinically significant difference, namely, $99.8 \%$ to detect a 1.0-J difference, should one have existed. As would be expected, lengthening the electrodes did significantly decrease the impedance $(P<0.05)$. Somewhat surprisingly was the fact that the longer electrode in the coronary sinus resulted in a lower impedance than having the longer electrode in the right atrium $(\mathrm{P}<0.05)$. We also evaluated the difference in the defibrillation threshold at the beginning of the study, when the episode was longer lasting (in all but one patient were atrial fibrillation was induced) to the threshold for subsequent induced episodes. We found that the initial threshold was not signifi- 
cantly higher $(\mathrm{P}>0.05)$ and even tended to be slightly lower. Additionally, comparing the threshold for the first trial to the last and normalizing for the difference in lengths used, no difference was seen $(P>0.05)$. A stable defibrillation threshold was seen in this study despite the delivery of multiple shocks, $31 \pm 8$ (range 20 to 44), and despite multiple reinductions of atrial fibrillation. Despite the muluple shocks and reinductions, there were no acute or long-term complications observed in the patients undergoing this study.

\section{DISCUSSION}

We found the technique of internal cardioversion to be quite effective at restoring sinus rhythm, even in patients who have falled previous atrempts at external defibrillation or who have long-standing drug refractory atrial fibrillation. Our results are similar to those reported by others 10,11 showing that these parients have higher thresholds than the thresholds reported in parients with induced or paroxysmal atrial fibrillation. ${ }^{1,2}$ However, unlike previously reported studies, ${ }^{12}$ we did nor find the threshold for the initial, long-standing atrial fibrillation episode to be higher than subsequent induced episodes. Our results are more consistent with the findings of Lau and Lok, who found that thresholds, if anything, rended to go upward for subsequent induced episodes. ${ }^{13}$ However, comparing the last threshold to the first threshold, there was no significant difference obserwed $(\mathrm{P}>0.05)$ despite the fact that a mean of 31 shocks were delivered.

The new finding in our study was that longer electrodes did not result in a lowering of defibrillation thresholds. Theoretically, one might expect that longer electrodes could lower thresholds by 1 of 2 mechanisms or both. The first mechanism is that longer electrodes might better encircle the atria, which may, in turn, result in a larger and more homogenous electrical field distribution. With a more homogenous field, more cells could be directly affected by the shock with less energy "wasted" in the area of highest field density, the area in direct apposition to the elecrodes. The second mechanism is that longer electrodes would lower the impedance of the shock pathway, thereby allowing more current to flow through the cells. Indeed, in our study, the impedance was significantly lowered with the longer elecrode lengths; however, this lowering in impedance did not correlate with a lowering in the defibrillation threshold. One possible explanarion for the results of this study is that current was actually shunted between the electrodes through the blood, as the coronary sinus lead may be too close to or slightly extending our of the os, allowing for easier shunting of curent through the blood and away from the cells at the distal end of the coronary sinus electrode. As the current is selecting the path of least resisrance and is flowing through the blood and not through the myocardial cells, the current for defibrillation may actually be diminished, offserring the potential benefit of the longer electrodes. Clinically, the catheters were posicioned in this study as 
distally as possible in the coronary sinus to attempe to mimic the dinical use of longer electrodes.

Despite the fact that previous studies have shown that increasing the electrode length to $6 \mathrm{~cm}$ from $3 \mathrm{~cm}$ lowered atrial defibrillation requirements, 6.7 wich might predict that yer longer electrodes would further reduce defibrillation requirements, our study showed this not to be the case. This finding has significant clinical implications in the design of temporary catheters, such as those used in this study, for the clinical conversion of atrial fibrillation episodes, but also for the design of permanent leads for an implantable atrioverter. Leads, however, more importantly catheters, with longer electrodes are more difficult to build and more difficult to position in the distal coronary sinus as the electrode material reduces the ability to curve these catheters and to position them properly.

Finally, we observed that, after restoration of sinus rhythm, one patient had a pause-dependent recurrence of atrial fibrillation. Stable sinus rhythm could be obtained in this patient using biatrial pacing in the postshock period. Wherher atrial pacing postcardioversion may be clinically useful in achieving stable sinus rhythm in other parients requires further evaluation.

In conclusion, we have confirmed that the technique of internal cardioversion using catheter-based electrodes positioned in the coronary sinus and right atrium is highly effective in restoring stable sinus rhythm at low energies. Despite the occasional recurrence of atrial fibrillation nearly immediately after successful termination of the same arrhythmia, stable sinus rhythm could be obtained as the clinical outcome in all patients in this study. Finally, longer electrode lengths do not lower thresholds for successful atrial defibrillation when the right atrium to coronary sinus shock vector is used.

\section{LIMITATIONS OF THE STUDY}

One potential limitation of this study is that the longer electrodes were generated by using rwo catheters, rather than a single catheter. This limitation was due to a practical reason, namely, that placing more electrodes on a carherer would substancially limit its flexibility and porentially prevent one from positioning the electrode as distally in the coronary sinus or prevent its conformation to the shape of the right atrium.

The second limitation was that the electrodes used here were not continuous coil electrodes but discrete solid electrodes that were connected together. This limitation was again caused by the practical issue that multipolar catheters are easier to obtain, as well as that the catheters could be positioned in the two locations without the need to reposition the $6-\mathrm{cm}$ portion when switching to the longer electrode length. 


\section{REFERENCES}

1. Murgatroyd FD, Slade AKB, Sopher SM, et all: Efficacy and tolerability of transwenous low energy cardioversion of paroxysmal atrial fibrillation in humans. I Am Coll Cardiol 1995:25:1347-1353.

2. Levy $\$$, Ricard 1 . Lau $C$, et alli Multicenter low energy transwenous atrial defibrillation (XAD) trial results in different subsets of atrial fibrillation. J Am Coll Cardiol 1997;29:750-755.

3. Schmitr $\mathrm{C}$, Alt E, Plewan $\mathrm{A}$, et al Low energy intracardiac cardioversion after failed conventional exrernal cardioversion of atrial fibrillation. I Am Coll Cardiol 1996:28:994-999.

4. Cooper RAS, Afferness CA, Smith WM, all: Internal cordioversion of atrial fibrillation in sheep. Circulation 1993:87:1673-1686.

5. Johnson EE, Yarger MD, Wharron JM. Monophasic and biphasic waveforms for low energy internal cardioversion of atrial fibrillation in humans. (Abstract) Circulation 1993;88:1-592.

6. Oriz J. Niwano $S$, Abe $H_{3}$ er al: Transvenous atrial defibrillation in two canine models of attial fibrillation. (Abstracr) I Am Coll Cardiol 1994;23:125A.

7. Hillsley RE, Yarger MD, Greenfeld RA, et al: Effect of catherer electrode length on internal atrial defibrillation thresholds in thumans. (Abstract) Circulation 1995;92:1-473.

8. Halperin BD, Reynolds B, Fain ES, er al: The effect of electrode size on transvenous defibrillation energy requirements: a prospecrive evaluation. PACE 1997;20:893 898.

9. Ayers GM, Alferness CA. Ilina M1, et al: Ventricuar proarthythmic effects of ventricular cycle length and shock strength in a sheep model of transwenous arrial defibrillation. Circulation 1994;89:413-422.

10. Alt E, Schmitt $C$, Ammer $R$, er al: Initial experience with intracardiac atrial defibrillation in patients with chronic arrial fibrillation. PACE 1994;17:1067-1078.

1 I. Lévy S, Ricard P. Gueunoun M, et al: Low-energy cardioversion of spontaneous atrial fibrillation. Immediate and long-term results. Circulation, 1997;96:253-259.

12. Santin $M$. Pandozi $C$, Toscano S, et al: Changes in intracardiac atrial cardiowersion threshold at rest and during exercise. J Am Coll Cardiol 1997;29:576-581.

13. Lat CP and Lok NS. A comparison of transwenous atrial defibrillacion of acute and chronic atrial fibrillation and the ffect of intravenous sotalol on human atrial defibrillation threshold. PACE $1997: 20: 2442-2452$. 
CHAPTER

\section{Decreasing the number of leads required for an implantable atrial defibrillator: use of a new 2-lead system}

Carl Timmermans", MD; Luz-Maria Rodriguez", MD; Gregory M Ayers", MD; Sandrine Masser ${ }^{* * *}$, MSc; Dawid G Reuter" MD; Hein J] Wellens", MD

From the "Department of Cardiology, Academic Hospital Mastricht. Maastricht. The Netherlands; and ** InConrrol Inc, Redmond, USA.

Abstract presented at the $21^{\text {st }}$ Annual Scientific Session of the Cangress of the European Society of Cadiolagy (Eur Heant J 1999:20,108) Accepted for potblication in Am Heart Joumal 


\section{A BSTRACT}

Background. A previous study with the implantable atrial defibrillator the Atrioverter, required the use of 3 leads. The purpose of this study was to evaluate the use of a new 2-lead system for detection of atrial fibrillation (AF) and atrial defibrillation.

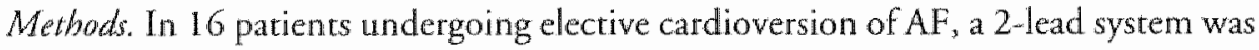
compared to the conventional 3-Jead system in a randomized trial. The new 2-lead system consisted of catheter with a distal bipolar right ventricular electrode pair and a proximal right atrial shock electrode coil and a separate decapolar defibrillation catheter in the coronary sinus. For the 3-lead system, a second decapolar catheter was placed in the right atrium. AF and sinus rhythm signal amplitude detection and atrial defibrillation threshold (ADFT) were compared in each patient using both systems.

Results. Successful defibrillation was obtained in all patienrs. ADFT for the 2-lead system (mean $370 \pm 112 \mathrm{~V}, 9.3 \pm 5.2 \mathrm{~J}$ ) was significantly higher compared to the 3 -lead system (mean $316 \pm 100 \mathrm{~V}, 6.8 \pm 4.2 \mathrm{~J} ; \mathrm{P}<0.05)$. In contrast, there was an increase in impedance for the 3 -lead system $(77 \pm 16 \Omega$ vs $68 \pm 13 \Omega, P<0.05)$. The mean right atrium-coronary sinus signal amplitude was $1.7 \pm 0.8 \mathrm{mV}$ during sinus thythm and $0.86 \pm 0.17 \mathrm{mV}$ during $A F$ in the 2-lead system. For the 3 -lead system, the right atrium-coronary sinus signal amplitude was $2.54 \pm 1.07 \mathrm{mV}$ and $1.59 \pm$ $0.53 \mathrm{mV}$ respectively. AF had a lower signal amplitude as compared to sinus rhythm for both systems $(P<0.05)$ and the 2 -lead system had a lower signal amplitude as compared to the 3 -lead system for both rhythms $(\mathrm{P}<0.05)$.

Conctustom. The use of a 2-lead system with this configuration is not superior to the 3-lead system with respect to $A F$ signal amplitude detection and ADF $\Gamma$. Further study is needed with implantable-quality leads in place of the temporary catheters used in this study. 
Catheter-based internal cardioversion of atrial fibrillation (AF) has been successfully performed in humans. 1-6 $^{-6}$ The safery and efficacy of this new method of cardioversion has resulted in the development of the Atrioverter. The Atrioverter has been used in an initial clinical trial of safery and efficacy. ${ }^{7}$ The system uses three leads, one in the coronary sinus, one in the right atrium and one in the right ventricle. The leads in the coronary sinus and right atrium are used by the Arrioverter for both detection of AF and for defibrillation shock delivery. The right ventricular lead has a bipolar pair of elecrrodes used for synchronization and posrshock pacing. Successful efforts were made to reduce the number of leads required by the Atrioverter by combining the right atrial and coronary sinus defibrillation electrodes on the same lead. ${ }^{810}$ Another 2-lead system concept would be to combine the right ventricular bipolar electrodes and the right atrial defibrillation electrode on the same lead. If such an approach would funcrion adequately for the Atrioverter, it would provide another oprion to decrease the required number of leads. We therefore evaluated this 2-lead system in comparison to the conventional 3-lead system with respect to AF signal amplitude derection and atrial defibrillarion threshold (ADFT).

\section{METHODS}

\section{STUDY POPULATION}

Sixteen consecurive patients with AF were prospectively studied. Fifteen of the parients underwent elecrive internal atrial defibrillation of $\mathrm{AF}$ and in 1 patient, $\mathrm{AF}$ was induced at time of study. Evaluation of the patients included a clinical history, physical examination, routine laboratory and thyroid function tests, a 12-lead ECG and transthoracic echocardiography.

Patients were excluded from the study if they met any one of the following criteria: 1) a left ventricular ejection fraction of less than $20 \%$ or uncompensated heart failure, 2) a previous history of a sustained ventricular arthythmia, cardiac arrest, congenital long QT syndrome, or anterograde conduction over an accessory parhway, 3) AF due to reversible causes (e.g., hyperthyroidism), 4) unstable angina in the past week, a history of myocardial infarction or a revascularisation procedure within the past four months, 5) digitalis toxicity or significant electrolyte imbalance or 6) an implanted pacing device.

The administration of antiarthythmic drugs was not interrupted for the procedure. Anticoagulation was interrupted 2 days before the procedure and restarted after the procedure. To perform a safe venous puncture an INR value of 2.5 was required. The study protocol was approved by the Medical Erhics Commitree of the Academic Hospiral Maastricht, and informed consent was obtained from all parients. 
A specially designed 6-French catheter (VascoMed, Institut fur Kathetertechnologie GmbH, Weil am Rhein, Germany) with a distal bipolar electrode pair and a proximal $6 \mathrm{~cm}$ electrode coil (surface area $5 \mathrm{~cm}^{2}$ ) was inserted through the left brachial vein ( $\mathrm{N}=13$ patients) or the right femoral vein ( $\mathrm{N}=3$ patients). In those patients in whom a brachial approach was used, the cartheter was positioned using a long vascular shearh (Daig Corp., Minnetonka, MN, USA), with the tip in the apex of the right ventricle. The catheter had three possible spacings $(11,15$, and $20 \mathrm{~cm})$ from the proximal electrode of the bipole to rhe distal part of the coil electrode. The size of the spacing was optimized on a per patient basis with an atrempt made to select the spacing that would result in positioning the coil electrode in the mid-lateral right atrium, for both the femoral and the antecubital approach. A temporary 6-French catheter (Elecath, Electro-Catheter Corp., Rahway, NJ, USA) consisting of 10 parallel stainless steel rings (surface area $3,12 \mathrm{~cm}^{2}$ ) was inserted into the right femoral vein and positioned in the distal coronary sinus. For the 2-lead system, the right atrium-coronary sinus detection signals were obtained and shocks were delivered between the decapolar carherer in the coronary sinus and the shock coil in the right atrium. For the 3-lead system, a second decapolar catheter was positioned in the high right atrium. The right atrial electrodes for both systems were positioned to attempt to achieve the best shock vector. The right atrial coil electrode was covered with the sheath and was disconnected, isolating it from the shock. The right atrium-coronary sinus detection signals were obtained and shocks were delivered between the two decapolar catheters (Figure 1). For both systems, shocks were synchronized and postshock pacing was performed using the bipolar pair of electrodes in the right ventricle.

Defibrillation shocks were delivered by the Metrix defibrillation system analyzer (model 2102, InControl Inc., Redmond, WA, USA) unless greater than $400 \mathrm{~V}$ (approximately $10 \mathrm{~J}$ ) was needed for defibrillation. For higher shock strengths, an external defibrillator (Ventritex HVS-02, Sunnyvale, CA, USA) was used. Biphasic defibrillation shocks ( $6 / 6 \mathrm{msec}$ ), synchronized to the $R$ wave, were given. To prevent inadvertent induction of ventricular fibrillation, shocks were delivered only after RR intervals longer than $500 \mathrm{msec}^{.1}$ In 1 patient AF was induced by right atrial burst pacing.

ADFT's were determined in a randomized order in each patient twice for each system using a $180 \mathrm{~V}$ start, $40 \mathrm{~V}$ step-up protocol until cardioversion of AF occurred. After successful shocks, AF was reinduced using rapid atrial pacing and allowed to sustain for 5 minutes prior to the next shock being delivered. Conscious sedation was provided using intravenous midazolam $(0.1 \mathrm{mg} / \mathrm{kg})$. Oxygen saturation was monitored using a pulse oximeter and the blood pressure was measured every 15 minutes. During the study, the 12-lead ECG and endocardial electrograms were 
A

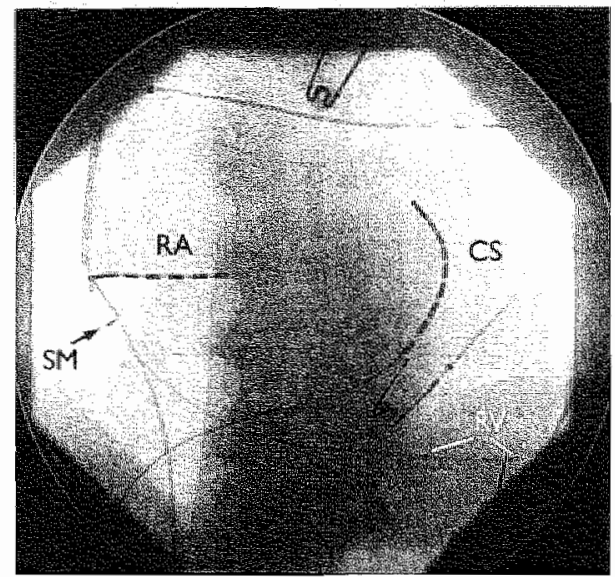

B

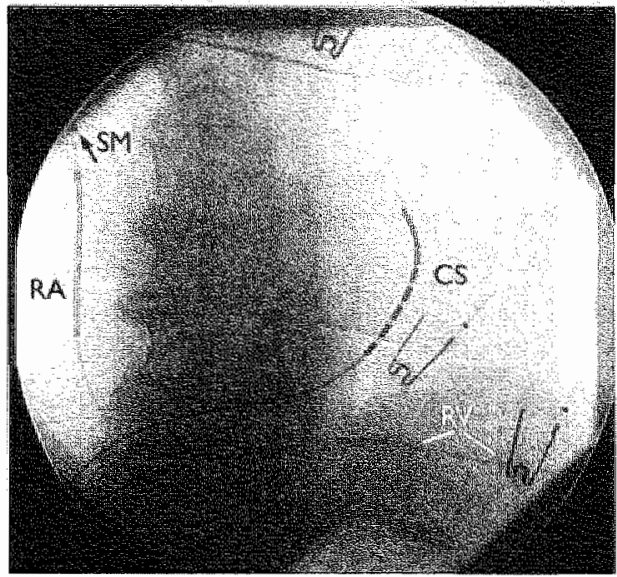

Figure 1 - Anteroposterior thoroscopic projection of the position of the defibrillation catherers for the two atrial defibrillation lead systems. In Figure IA, a 3-lead system is shown. The sheath marker (SM) is positioned distal to the right atrial (RA) coil electrode, so that the sheath is covering this coil electrode. The distal bipolar elecrode pair of the carheter is located in the right ventricular apex (RV). A decapolar defibrillation catherer is added in the high RA. A coronary sinus (CS) decapolar defibrillation catheter is located distal in the CS. In Vigure $1 \mathrm{~B}$, a 2-lead sysrem is shown in the same patient. Note that the shearh is withdrawn from the RA coil dectrode.

recorded and stored by PC-EMS 3.0 (Cardiovascular Research Institute, Maastricht, The Netherlands).

AF detection signals (right atrium-coronary sinus) were obrained using the automatic gain control algorithm of the Metrix defibrillation system analyzer. The signals across the shock vector were analyzed at two different sense margin ratios for each lead system and for both sinus rhythm and AF. The automatic gain control algorithm uses the four largest atrial deflections seen in 8 seconds of the detecrion electrogram to set the sensitivity. The amplitude of the signals was calculated from the sense margin ratio set and the resultant sensitivity provided by the system. ${ }^{12}$

\section{STATISTICAL ANALYSIS}

Continuous variables are expressed as mean values \pm SD. ADFT (voltage, energy) and impedance for the two lead systems were compared using Student's paired $t$-test. Order effects were evaluated by using the testing order as a covariate. Comparison of signal amplitude detections was made using a two-way mixed model analysis of variance. $P<0.05$ was considered statistically significant. 


\section{RESULTS}

\section{CLTNICAL CHARACTERISTICS}

Table I gives the clinical and echocardiographic characteristics of the parients studied. Twelve of the 16 patients had previously failed 1 or more attempts at external cardioversion. In general, the patients had a long history of AF with associated changes in left atrial diameter.

Table 1.- Clinical and echocardiographic characteristics of the patients studied.

\begin{tabular}{|c|c|}
\hline Men Jwomen & $14 / 2$ \\
\hline \multicolumn{2}{|l|}{ Age (years) } \\
\hline$M e a n+S D$ & $54+9$ \\
\hline Range & $39-76$ \\
\hline \multicolumn{2}{|c|}{ Duration $A$ as discase (montha) } \\
\hline Mexn $\mathrm{SD}$ & $50.8 \pm 45.8$ \\
\hline Range & $6 \cdot 144$ \\
\hline \multicolumn{2}{|c|}{ Durarion rieacd Al episode (days) } \\
\hline$M \operatorname{ann} \pm S \mathrm{D}$ & $475+290^{\circ}$ \\
\hline Range & $0.13-1.953$ \\
\hline
\end{tabular}

Current antiarthythmic elug

Class I 2

Class II 5

Class 1111

Class IV 0

Combination of anriarnythmic drugge

No antantaythmic drug 2

1.A dianerertimn)
Merain $5 \mathrm{SD}$
$54: 5$
Range.
$42-60$

L. $V \mathrm{E}, \mathrm{O})$

Mete $\mathrm{SD}$

$53 \pm 1$

$34-72$

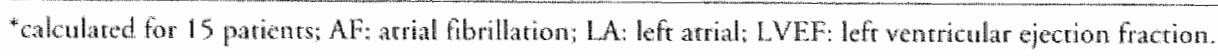



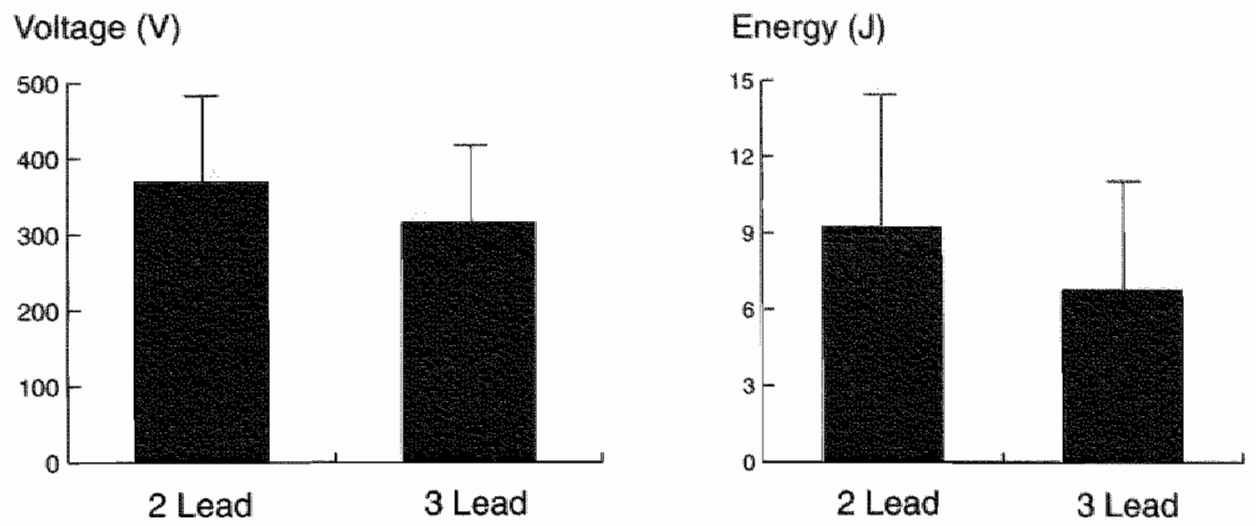

Figure 2. - Atrial defibrillation thesholds (voltage in left panel, energy in right panel) for the rwo lead systems. Shown are dara expressed as mearn with SD.

\section{OUTCOME OF INTERNAL ATRIAL DEFIBRILLATION AND COMPARISON OF LEAD SYSTEMS}

All patients were successfully cardioverted with both lead systems and all patients completed the entire protocol. The mean ADFT and impedance for the 2-lead system was $370 \pm 112 \mathrm{~V}, 9.3 \pm 5.2 \mathrm{~J}$ and $68 \pm 13 \Omega$. For the 3 -lead system, these values were $316 \pm 100 \mathrm{~V}, 6.8 \pm 4.2 \mathrm{~J}$, and $77 \pm 16 \Omega$, respectively. Comparing threshold voltage and energy for the two lead systems showed a significantly lower threshold for the 3-lead system $(\mathrm{P}<0.05$, Figure 2). Conversely, the impedance for the 3-lead system was significantly higher $(P<0.05)$. We tested whether the order of testing had any effect on the threshold comparison and found that there was no ordering effect ( $>0.05)$. On a per parient basis, the 3-lead system had lower energy requirements for 9 patients (average decrease of $5.3 \mathrm{~J}$ ) and was within $1 \mathrm{~J}$ of the 2-lead system for 5 patients. In only 2 patients were the energy requirements lower for the 2-lead system (average decrease of $4.2 \mathrm{~J}$ ).

The results of the comparison of the signal amplitude detecrion (right atrium-coronary sinus) for both lead systems during $\mathrm{AF}$ and sinus rhythm are shown in Figure 3. For both lead systems, AF had a lower signal amplitude as compared to sinus rhythm ( $<0.05)$. For both rhythms, the signal amplitudes were greater for the 3 -llead system as compared to the 2 -lead system $(P<0.05)$.

All patients were in stable sinus rhythm at the end of the procedure despite the fact that 6 of the parients had immediate reintriation of Af at some time during the procedure. 13 Despite the multiple shocks and reinducrions, there were no acute or long-term complications observed. 


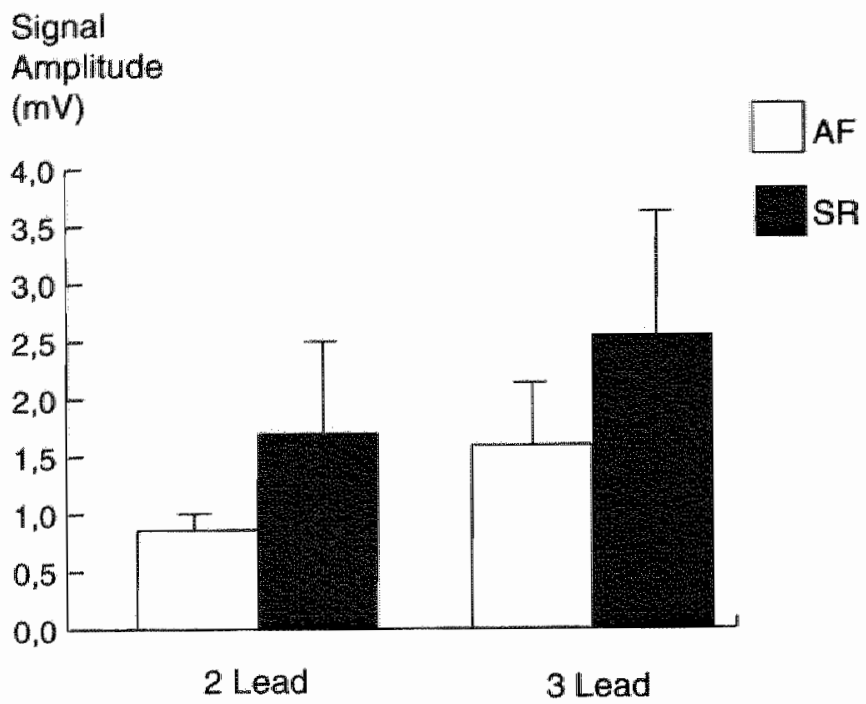

Figure 3. - Right atrium to cononary sinus signal amplitude derection for borh lead systems during arrial fibrillation (AF) and sinus thythm (SR). Shown are data cxpressed as mean with SD.

\section{DISCUSSION}

Internal atrial defibrillation was effective in restoring stable sinus rhythm in all patients, despite the long mean duration of AF and previously failed external atrempts. The high efficacy of the technique and the ADFT's found in this study are similar to the results of other studies. ${ }^{1-6}$ It seems that in patients that have failed external cardioversion attempts, it is worthwhile to attempt internal defibrillation for the restoration of sinus thythm. We did not find a significant order effect, indicating that the first threshold terminating an episode of long standing AF, was not significantly different from later thresholds, determined after induced AF episodes. 4,6

The main finding in our study was that the presently evaluated 2-lead system was inferior to the conventional 3-lead system with respect to defibrillation threshold and signal amplitudes for $\mathrm{AF}$ detection. One must consider that the 2-lead system offers greater simplicity, potentially less morbidity and fewer lead dislodgements. However, ir seems that the higher thresholds would make its utility lower. Previous studies focusing on the use of a combined coronary sinus-right atrial lead (Solo lead) showed this system to be equivalent to the conventional 3-lead system. ${ }^{8,9}$ The reason for the difference is likely related to the position of the right atrial electrode. In the 2-lead system in our study, where one lead contains the right atrial shock electrode and the right ventricular pace/sense electrodes, the location of rhe right atrial. elec- 
trode is variable and not always in contact with the lateral aspect of the atrium. Furthermore, in 3 parients, the catheter was positioned from the right femoral vein making a loop in the right atrium to simulate the situation when the catheter was introduced by way of the left brachial vein. This would also occasionally cause the coil to be seprally located. "The Solo lead, when exiting the os of the coronary sinus, forces the right atrial electrode towards the lateral wall of the right atrium. The deflection point for the right atrial part of our 2-lead system was the tricuspid valve making the floaring coil position more variable and less stable than the Solo system. Another measure of optimal location was the impedance. If the impedance is higher, it is likely that more tissue is present between the defibrillation elecrrodes. The efficacy of defibrillation relies on developing an adequate field strength in a critical mass of cells. Having less tissue between the electrodes will make it less likely to develop a field strength necessary to defibrillate the critical mass. As the impedance is lower for the 2-lead system, it is likely that less mass of tissue is between the electrodes and therefore a larger voltage is required to develop an adequate defibrillating current across the cells. Impedance can also be affected by electrode material and surface area, however a previous study has shown the coil electrode to be similar in impedance, with the coil electrode having a slightly lower impedance. ${ }^{14}$ It is possible that the lower impedance in our study was due to the coil electrode used in the 2-lead system.

As with the Solo system, variable interelectrode spacing was necessary to attempt to locate the coil within the right atrium. Even with the choice of three interelectrode spacings, we often found it difficult to position the right atrial electrode optimally. An advantage of this 2-lead system over the Sollo system is that the Solo system is dependent on depth of coronary sinus penetration. In either case, it is clear, that the possibility of multiple interelectrode spacings will be required for success of a 2-lead system. The 3-lead system allows greater flexibility because all 3-leads are independently positioned. For example, in patients where the coronary sinus is not cannulated deeply, the right atrial electrode in a 3-lead system can be independently positioned to optimize irs location in spite of a less optimal coronary sinus location.

In conclusion, we have shown that a 2-lead system, with one lead combining the right ventricular bipolar and right atrial coil electrode, although able to convert AF in all patients, could not compete with the conventional 3-lead system. Additionally, the detection signals from the 2-lead system were of lower amplitude, potentially making AF detection more difficult or more sensitive to noise.

\section{LIMITATIONS OF THE STUDY}

One porential limitation of this study is that we attempted to mimic a chronically implantable lead with a temporary catheter. We were trying to evaluate the practical limitations of the rwo lead systems tested and attempted to optimize each lead system for the anatomy and vascular approach. There is a potential difference due to the different elecrrodes (coil vs. decapolar) of the two systems. However, a study by 
Elabbady, et al comparing a coil and temporary bipolar electrode in sheep showed no difference in defibrillation threshold or impedance. ${ }^{1 \frac{4}{4}}$ Additionally, the flexibility of this catheter and the long vascular course of the catheter from the arm required that we used a long vascular sheath to position this catheter. To awoid the need to replace the catheter, we had to cover the right atrial electrode when testing the 3-lead system. This sheath may have resulted in a somewhat unnatural curvature and a less optimal position of the catheter.

\section{REFERENCES}

1. Murgatroyd FD, Slade AKB, Sopher SM, et al. Efficacy and tolerability of transwenous low energy cardioversion of paroxysmal atrial fibrillation in humans. J An Coll Cardiol 1995:25:1347-53.

2. Levy $\$$, Ricard P, Lau CP, et al. Multicenter low energy transwenous atrial defibrillation (XAD) trial results in different subsers of atrial fibrillation. I Am Coll Cardiol 1997;29:750-5.

3. Alt E, Schmitt $C$, Ammer $\mathbb{R}$, at al. Effect of electrode position on autcome of low-energy intracardiac cardioversion of atrial fibrillation. Am J Cardiol 1997;79:621-5.

4. Lau CP, Lok NS A comparison of transvernous atrial defibrillation of acure and chronic arrial fibrillation and the effect of intrawenous sotalol on human atrial defibrillation threshold. Pacing Cin Electrophysiol 1997;20:2442-52.

5. Lok NS, Lau CP, Tse HF, et al. Clinical shock tolerability and effect of different right atrial electrode locations on eficacy of low energy human transvenous atrial defibrillation using an implantable lead system. J Am Coll Cardiol 1997;30:1324-30.

6. Timmermans C, Rodriguez LM, Ayers GM, et al. Effect of electrode length on atrial defibrillation thresholds. J Cardiovase Electrophysiol 1998;9:582-7.

7. Wellens HJ], Lau CP, Leideritz B, et al. Atrioverter: An implantable device for the treatment of atrial fiberillarion. Circulation 1998;98:1651-6.

8. Tse ME, Lan CP. Ayers GM. Initial dinical experience with a single-pass (Solo) dual electrode lead for an implanable atrial defibrillator. Pacing Clin Electroplysiol 1997;20(II):1126.

9. Tse HF, Lau CP, Ayers GM. The feasibility of a single-pass dual electrode lead (Solo) for atrial fibrillation detecrion in an implantable atrial defibritlator. Pacing Clin Electrophysiol 1998;21(II): 909.

10. Heisel A, Jung J, Neuzher J, en al. Low-energy transwenous cardioversion of arrial fibrillation using, a single atrial llead system. I Cardiovasc Electrophysiol 1997;8:607-14.

11. Ayers CM, Alferness $C A$. Ilina $M$, et al. Ventricular proarrhythmic effects of ventricular cycle length and shock strength in a sheep model of transwenous atrial defibrillation. Circulation 1994;89:413-22.

12. Kim J. Bocek J, Whire $H$, er al. An atrial fibrillation detection algorithm for an implantable atrial defibrillaror. Computers in Cardiology 1995:169-72.

13. Timmermans C, Rodriguez LM, Smeets JLRM, er al. Immediate reinitiation of atrial fibrillation following internal atrial defibrillation. I Cardiowasc Electrophysiol 1998;9:122-7.

14. Elabbady TZ. Tacker WA, Geddes LA, et al. Preimplant atrial defibrillation testing with a remporary catheter in sheep. Pacing Clin Electrophysiol 1997;20:1754-8. 
CHAP管

\section{Effect of butorphanol tartrate on shock related discomfort during internal atrial defibrillation}

Carl Timmermans, MD; Luz-Maria Rodriguez, MD; Gregory M Ayers, MD; Hendrik Lambert, PhD; Joep L.RM Smeets, MD; Johan WS Vlacyen, PhD;

Adelin Albert, PhD; Hein II Wellens, MD

From the Department of Cardiology (C.T., L.M.R., J.L.R.M.S., H.J.J.W.) and Pain Management and Research Center (J.W.S.V.), Academic Hospital Masstricht, The Netherlands; the Department of Biostaristics (A.A.), University of Liege, Belgium; and InControl Inc (G.M.A., H.L.), Redmond, USA.

Abstace presented in part at the $4^{\text {th }}$ Annual Scientific Session of the American College of Cardiology 1997 (J An Coll Cardiol 1997:29, 474A)

Published in Circulation 1999,99:1837-1842 


\section{A B S T A C T}

Background. In patients with atrial fibrillation, intracardiac atrial defibrillation causes discomfort. An casily applicable, short acting analgesic and anxiolytic drug would increase acceptability of this new treatment mode.

Methods and Results. In a double-blind placebo-controlled manner, the effect of intranasal butorphanol, an opioid, was evaluated in 47 patiencs with the use of a step-up internal atrial defibrillation protocol (stage I). On request, additional butorphanol was administered and the step-up protocol continued (stage II). Thereafter, if necessary, patients were intravenously sedated (stage III). After each shock, the McGill Pain Questionnaire was used to obtain a sensory (S), affective (A), evaluative (E) and total (T) pain rating index (PRO) and a visual analogue scale analyzing pain (VAS-P) and fear (VAS-F). For every patient, the slope of each pain or fear parameter against the shock number was calculated and individnal slopes were averaged for the placebo and butorphanol group. All patients were cardioverted at a mean threshold of $4.4 \pm 3.3 \mathrm{~J}$. Comparing both patient groups for stage II, the mean slopes for PRI-T (P=0.0099), PRI-S ( $\mathrm{P}=0.019)$ and PRI-E (P=0.015) became significantly lower in the butorphanol group than in the placebo group. Comparing patients who received the same shock intensity ending stage I and going to stage II, in those parients randomized to placebo the mean VAS-P (P=0.023), PRI-T $(P=0.029)$, PRL $-S(P=0.030)$ and PRI-E $(P=0.023)$ became significantly lower after butorphanol administration.

Conclwsions. During a step up internal atrial defibrillation protocol, intranasal butorphanol decreased or stabilized the value of several pain variables and did not affect fear. Of the 3 qualitative components of pain, only the affective component was not influenced by butorphanol. The PRI evaluated pain more accurately than the VAS. 
Although the amount of energy needed for internal cardioversion of atrial fibrillation is substantially reduced when using the right atrium-to-coronary sinus electrode configuration in combination with a biphasic shock waveform, intravenous administration of anesthetics is usually necessary to reduce the discomfort of patient shock. ${ }^{1-5}$ The anesthetic properties of some of these drugs and their intravenous mode of administration may diminish some of the advantages of internal cardioversion over external cardioversion. The shock-related discomfort has components of pain and fear. ${ }^{2}$ Many factors could contribute to the mechanism producing discomfort, including skeletal muscle contraction, nerve stimulation and psychological factors." The purpose of this study was to evaluate, in a double-blind placebo-controlled manner, the effect of a transnasally administered opioid analgesic (burorphanol tartrate) on patient-reported pain and fear during internal cardioversion of atrial fibrillation.

\section{METHODS}

\section{STUDY POPULATION}

Forty-seven consecutive patients with atrial fibrillation 29 men and 18 women; mean age $63 \pm 12$ years, range 28 to 85 ) in whom conversion to sinus rhyrhm was clinically indicated, underwent internal atrial defibrillation. Clinical evaluation of the patients included a history, physical examination, routine laboratory and thyroid function tests, a 12-lead ECG and transthoracic and transesophageal echocardiography. The exclusion criteria of this study have been described previously. ${ }^{6}$ The administration of antiarrhythmic drugs was not interrupted for the procedure. Anticoagulation was interrupted 2 days before the procedure and restarted after the procedure. The study protocol was approved by the Medical Ethics Committee of the Academic Hospital Maastricht, and written informed consent was obtained from all patients.

TRANSNASAL BUTORPHANOL

Butorphanol tartrate (Bristol-Myers Squibb) is a synthetic opioid analgesic of the phenanthrene series. After intranasal administration, onset of analgesia occurs within 15 minutes with a peak effect at 30 to 60 minutes after a 1 -mg dose. The elimination half-life is 4.7 hours in individuals, ages 20 to 40 years, and 6.6 hours in individuals older than 65 years. The recommended starting dose for nasal administration is $1 \mathrm{mg}$ ( 1 spray in 1 nostril) and a second 1 - $\mathrm{mg}$ dose may be given 60 to 90 minutes after the first dose.

The analgesic was supplied in a marker package containing $2.5 \mathrm{ml} \mathrm{of} \mathrm{a} 10 \mathrm{mg} / \mathrm{mI}$ solution of butorphanol tartrate. A solution of $0.8 \%$ sodium chloride was used as 
placebo. Drug and placebo were transerted to empry 2.5 -m botes by a pharmacist of the Academic Hospita Matricht, who also performed the randomization. Before each procedure. 2 boteles whthetered pumps $(0.09 \mathrm{~m} / \mathrm{stroke}$ ) were delvered: I labelled with and containing placebo or butorphanol, according to the randomization, for the frst intaxisal administration and 1 labelled with and containing butorphanol for a potential second administration.

\section{MEGILL PAIN QUESTIONMAIRE, DUTCH LANGUAGE VERSTON}

The McGill Pain Questioname (MPQ) is widely recognized as a wald and reliable instrument no measure pain, with a Dutch language version (MPQ-DLV) avalable. The MPQ-DLV contains 20 subclasses of 3 to 4 pain-descriptive words arranged in progressively increasing intensity. Twelve subclasses of 3 words describe sensory modalities of pain experience, 5 subclasses of 3 words represent affective aspects and 3 subclasses of 4 words represent the evaluative dimension of pain. The list of words is scored according to the method described by Melzack." Two major indexes are obtaned; the pain rating index. (PRI) and the number of words chosen. As shown by Vlaeyen, 10 the PRI and the number of words chosen appear to intercortelate highly. Therefore we included only the PRI for further analysis. The PRI is based on the rank values of the words. The values of the words chosen by a patient are then added up to obtain a scote separaty for the sensory (PRL-S), affective (RRL-A) and evaluarive (PRL-E) words, in addition to providing a total score (PRI-T).

All patients were instructed on their participation in answering the questions of the MPQ-DLV before the start of the procedure. After each shock, except when the patient was intravenously sedated, the 63 pain-descriptive words of the 20 subclasses were read to the parient. The patient could only select from each subclass the word describing the sensation at that moment. If in a subclass none of the words represented his pain, nothing was recorded and the examiner proceded to the next group of wonds. Besides the list, 2 wisual analogue scales (VAS), 1 for quantification of pain. (VAS-P) and $\mathbb{1}$ for quantification of fear (VAS-F) were completed after each defibrillation shock. The VAS were numbered from 0 to 100 ; 0 represented no pain or foar (VAS-F) and 100 represented the maximum score for pain or fear during shock delivery. The PRI of the 3 individual pain dimensions (sensory, affective and evaluative) and their total were calculated from the parient answers for each shock delivery.

\section{INTERNAL ATRTAL DEFIBRTLLATION PROTOCOL}

Two 6F defibrillation catheters (Elecath, Electro-Catheter Corp) were inserted into the right femoral vein and positioned with 1 in the anterolateral right atrium and 1 in the coronary sinus. With the right atrial catheter used as the carhode and the coronary sinus catheter as the anode, a $6 / 6$ msec biphasic defibrillation shock, synchrom 


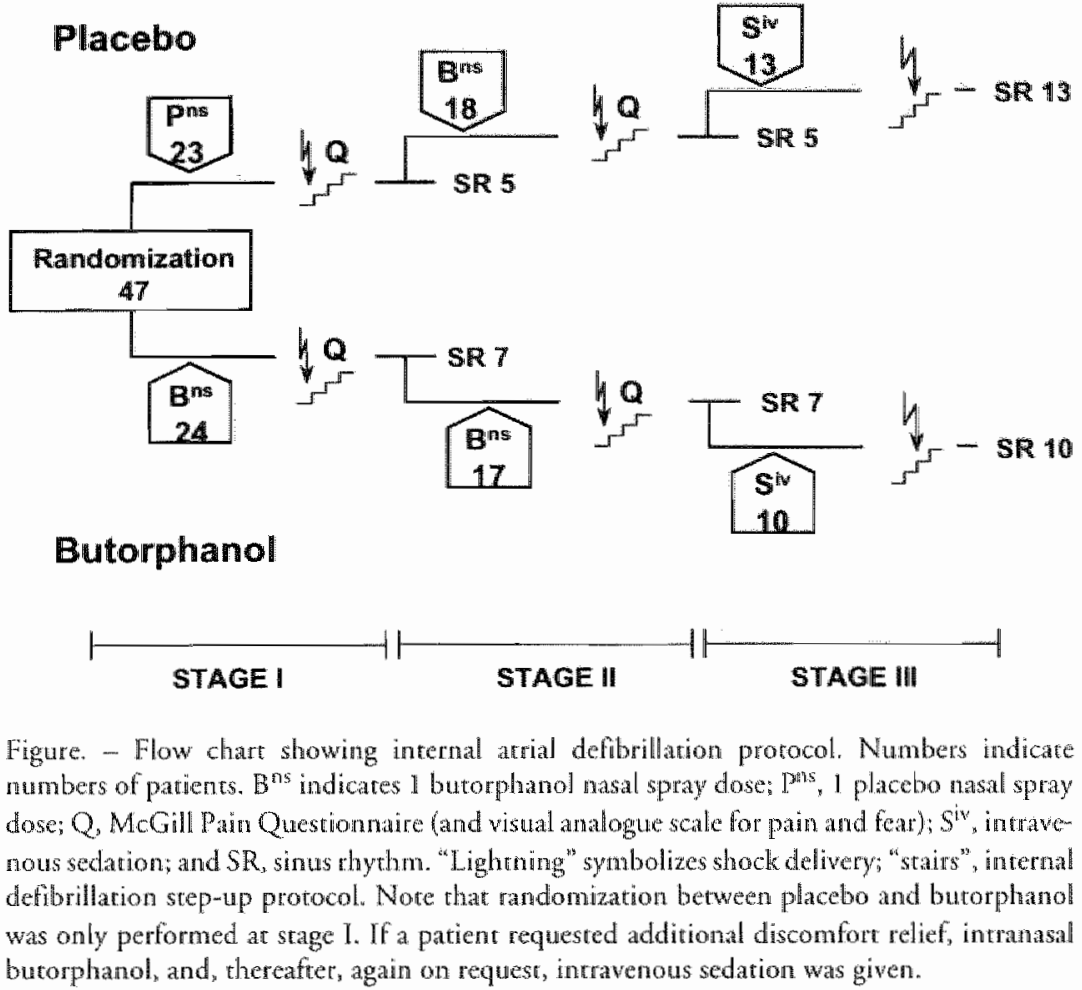

nized to the R-wave, was delivered from an external defibrillator (Ventritex HVS-02). To prevent inadvertent induction of ventricular fibrillation, shocks were delivered only after R-R intervals $>500 \mathrm{~ms} .{ }^{11}$ A quadripolar catheter (USCl) was positioned at the right ventricular apex to allow temporary ventricular postshock pacing.

Once all catheters were in position, 1 dose of nasal spray of either placebo or butorphanol was administered in 1 nostril (srage I; see Figure). Randomization between placebo and butorphanol was only performed at stage l. After 30 minutes, a $90-\mathrm{V}$ (approximately $0.5 \mathrm{~J}$ ) shock was delivered from the Ventritex HVS-02. If the shock was unsuccessful at converting atrial fibrillation and if the patient agreed to have a stronger intensity shock delivered, a 0.5 - ] step-up protocol until 3. J and thereafter a 1-J step-up protocol was followed until successful cardioversion was obtained or until the patient requested discomfort relief. Five minutes was allowed for the next shock to be delivered. After each shock was delivered, the patient was asked the level of discomfort by use of the MPQ-DLV and the VAS for pain and fear. If the patient requested additional medication for discomfort, he or she received 1 dose of butorphanol (1 mg) nasal spray in the other nostril (stage II). After 30 minutes, the previous shock intensiry was repeated to obtain paired shock intensiry-discomfort perceprion dara in the same patient and the step-up protocol continued until 
successul cardioversion was obtained or until the patient again requested discomfort relief. On the basis of the clinical situation, intravenous etomidate $(0.2 \mathrm{mg} / \mathrm{kg})$ or midazolam $(0.1 \mathrm{mg} / \mathrm{kg}$ ) was then given and shocks delivered until successful cardioversion was obtained (stage III). The defibrillation threhold was defined as the lowest shock energy (voltage) that converted atrial fibrillation into sinus thythm. After each defibrillation, the voltage and the impedance of each phase of the biphasic waveform were recorded from the external defibrillator and the total delivered enurgy was computed.

\section{STATISTICAL ANALYSIS}

On the basis of the data of a pilot study, ${ }^{12}$ it was determined that a sample size of 41 patients would be required to detect a $25 \%$ reduction in the VAS-P $(\alpha=0.05$. $\beta=0.20$ ) at the same shock intensicy comparing the on drug/off drug states (assuming the worst case of no correlation between the placebo and drug response). Furthermore, if the drug permitted the delivery of a shock at an intensity of $150 \%$ without significantly increasing the VAS-P, this difference would, as well, be detected with a sample size of $46(\alpha=0.05, \beta=0.20)$. Therefore a minimum of 46 parients were to be enrolled.

Results are expressed as mean $\pm \mathrm{SD}$ or SEM. Comparison of mean values was performed with the non-parametric Wilcoxon rank sum test for unpaired samples and with the Wilcoxon signed-rank test for paired observations. Proportions were compared with the use of the Chi-square test for contingency tables. Linear regression was used to calculate the slope of each variable (VAS, PRI) on the corresponding shock number for each patient. A positive slope indicated an increase in pain or fear when more shocks with increasing intensity were administered to the patient. Individual slopes were averaged for the placebo and butorphanol group during stages I and II of the study. All results were considered to be significant at $\mathrm{P}<$ 0.05. Statistical calculations were performed with SAS (SAS software version 6.11) and S-PLUS (S-PLUS software version 3.1, StatSci Europe) statistical packages.

\section{RESULTS}

\section{CLINICAL CHARACTERISTICS}

Of the 47 enrolled patients, 24 were assigned to butorphanol and 23 to placebo on the basis of inttial randomization. The clinical and echocardiographic characteristics of borh parients groups are reported in Table 1. No significant differences were found between the groups according to sex, age, durarion of arrial fibrillation as disease, duration of treared arrial fibrillation episodes, presence of structural heart disease, use of antiarthythmic drugs, left atrial diameter and left ventricular ejection fraction. 
Table 1. - Clinical and echocardiographic characteristics of patients randomized to butorphanoland placebo.

\begin{tabular}{lll}
\hline & $\begin{array}{l}\text { Butorphanol group } \\
(n=24)\end{array}$ & $\begin{array}{l}\text { Placebogroup } \\
(n=23)\end{array}$ \\
\hline Men/women & 14110 & $15 / 8$
\end{tabular}

Age (years)

$\begin{array}{lll}\text { Mean } \pm S D & 61 \pm 14 & 66 \pm 11 \\ \text { Range } & 28-84 & 47-85\end{array}$

Duration $A F$ as disease (years)
Mean \pm SD
$7.2 \pm 6.2$
$5.6 \pm 5.9$
Range
$0.25-23$
$0.04-20$

Durarion treated AF episode (days)
Mean \pm SD
$300 \pm 495^{*}$
$235 \pm 271+$
Range
$3 \cdot 2100$
$3-1000$

Structaral heart disease

$\begin{array}{lll}\text { Ischemic } & 5 & 4 \\ \text { Valvular } & 3 & 2 \\ \text { Cardiomyopathy } & 1 & 2 \\ \text { Hypertensive } & 9 & 6 \\ \text { Congenital } & 1 & 0 \\ \mathrm{AF} & 5 & 9\end{array}$

Current antiarnythmic drug

$\begin{array}{lll}\text { Class I } & 1 & 0 \\ \text { Class II } & 0 & 3 \\ \text { Class III } & 9 & 8 \\ \text { Class IV } & 1 . & 2 \\ \text { Digiralis } & 13 & 10 \\ \text { Combinarion of antiarrhychmic drugs } & 9 & 4 \\ \text { anriarrhythmic drug } & 1 & 2\end{array}$

LA diameter (mm)
Mean \pm SD
$53 \pm 7$
$53 \pm 5$
Range
$42-70$
$42-60$

LVEF (\%)
Mean 1 SD
$57 \pm 11$
$54 \div 12$
Range
$20-71$
$29-73$

AF: atrial fibrillarion; LA: left arrial; LVEF: left ventricular ejecrion fraction. Values are number of paticnts unless orherwise specified. *Value calculared for 20 patients. Talue calculared for 19 parients. 
All parients were successfully cardioverted at a mean threshold of $4.4 \pm 3.3 \mathrm{~J}(0.5$ to 15) or $258 \pm 94 \mathrm{~V}(90$ to 510$)$ without complications. No significant difference was found when parients were initially randomized to butorphanol or to placebo with regard to their mean defibrillation threshold ( $4.3 \pm 3.4$ vs $4.6 \pm 3.2$ ); $\mathrm{P}=0.76$ ). The mean number of shocks delivered to obtain sinus rhythm did also not differ between the butorphanol and placebo groups $(7.6 \pm 4.0 \mathrm{vs} 8.3 \pm 4.5$ shocks; $\mathbb{P}=0.58)$. In the butorphanol group 183 shocks were given and in the placebo group 192 shocks. The number of patients through the various stages of the study is shown in the Figure. In $24(51 \%)$ of 47 patients, sinus rhythm was obtained without the need for intravenous sedation, with a mean energy of $2.3 \pm 1.5 \mathrm{~J}$ (range 0.5 to 6.9 ) or a mean voltage of $190 \pm 59 \mathrm{~V}$ (range 90 to 340 ) and a mean number of shocks of $4.8 \pm 2.5$ (range 1 to 11 ). In contrast, in 23 (49\%) of 47 parients requiring intravenous sedation, successful cardioversion of atrial fibrillation occurred at a mean energy of $6.6 \pm 2.9 \mathrm{~J}$ (range 2.6 to 15.3) or a mean voltage of $329 \pm 68 \mathrm{~V}$ (range 210 to 510 ) and a mean number of shocks of $11.1 \pm 2.4$ (range 7 to 17 ).

There was no significant difference between the patient groups nor between successful and failed cardioversions for the average number of shocks delivered. However, for those patients undergoing both study stages, there was a significant decrease of the average number of shocks delivered going from stage I to stage II. In the placebo group, the average number of shocks decreased from 4.0 to 2.6 , whereas in the butorphanol group it decreased from 3.3 to 2.5 (Table 2). There was allso no significant difference between the patient groups nor between successful and failed cardioversions for the mean amount of energy delivered during the first 2 study stages (Table 3).

Table 2.- Mean number of shocks delivered according ro treatment, outcome of internal atrial defibrillation, and study stage.

\begin{tabular}{llll} 
Treament & Outcome & Srage I & Stage II \\
\hline Placebo & SR & $3.4 \pm 1.5(\mathrm{n}=5)$ & $2.6 \pm 1.7(\mathrm{n}=5)$ \\
& no SR & $4.0 \pm 1.9(\mathrm{n}=18)$ & $2.6 \pm 0.65(\mathrm{n}=13)$ \\
Butl & $3.9 \pm 1.8(\mathrm{n}=23)$ & $2.6 \pm 1.0(\mathrm{n}=18)$ \\
& SR & $3.9 \pm 1.4(\mathrm{n}=7)$ & $2.4 \pm 1.0(\mathrm{n}=7)$ \\
& no SR & $3.3 \pm 1.1(\mathrm{n}=17)$ & $2.6 \pm 1.5(\mathrm{n}=10)$ \\
& All & $3.5 \pm 1.2(\mathrm{n}=24)$ & $2.5 \pm 1.3(\mathrm{~m}=17)$ \\
\hline
\end{tabular}

SR: sinus thythm; n: number of patients. Vallues are mean $\pm S D$. 
Table 3. - Mean amoun of energy (in Joules) delivered according ro creatineme, outcome of internat atrial defibrillation, and study stage.

\begin{tabular}{llll}
\hline Treament & Ourcome & Stage I & Stage II \\
\hline Placebo & SR & $1.8 \pm 0.75(\mathrm{n}=5)$ & $3.4 \pm 2.5(\mathrm{n}=5)$ \\
& no SR & $2.2 \pm 1.5(\mathrm{n}=18)$ & $3.1 \pm 1.4(\mathrm{n}=13)$ \\
& All & $2.1 \pm 1.4(\mathrm{n}=23)$ & $3.2 \pm 1.7(\mathrm{n}=18)$ \\
Burorphanol & SR & $2.1 \pm 0.82(\mathrm{n}=7)$ & $2.5 \pm 1.3(\mathrm{n}=7)$ \\
& noSR & $1.6 \pm 0.54(\mathrm{n}=17)$ & $2.7 \pm 1.6(\mathrm{n}=10)$ \\
& Al & $1.8 \pm 0.65(\mathrm{n}=24)$ & $2.6 \pm 1.4(\mathrm{n}=17)$ \\
\hline
\end{tabular}

SR: sinus $\mathrm{El}$ ythm; n: rumber of patients. Values are mean \pm SD.

ANALYSIS OF PAIN AND FEAR VARIABLES DURING STAGES I AND II

Comparison of Placebo Group With Butompanol Growp for Stages I and II

All parients received a first shock of $90 \mathrm{~V}$ at the start of stage $\mathrm{I}$. There was no evidence of a VAS-P difference berween the placebo and butorphanol group ar $90 \mathrm{~V}$ $(\mathbb{P}=0.75)$, nor was there any evidence of a WAS-F difference $(\mathbb{P}=0.51)$, a PRI-T difference $(P=0.84)$, a $P R I-S$ difference $(P=0.77)$, a PRI-A difference $(P=0.34)$, or a PRI-E difference $(P=0.99)$.

For patients in stage $\mathbb{I}$, the slope of the regression line for each pain (except for PRI-A in patients randomized to butorphanol) and fear variable and the corresponding shock number was significantly positive, indicating that pain and fear increased with the number and the intensity of shocks received (Table 4). No significant difference was found between the placebo and butorphanol groups according to the mean slope of the different pain and fear variables during stage I. Nevertheless, there was a definite trend for PRI-T, PRI-S and PRI-A. During stage II, there was also no significant difference between both patient groups for the mean slope for VAS-F and PRI-A, whereas the mean slope for VAS-P showed a trend. The mean slopes for PRI-T, PRI-S and PRI-E, however, became significandy lower in the butorphanol group than in the placebo group. Furthermore, during stage II, the slope for VAS-P and the 4 PRI slopes were no longer significantly different from zero in the butorphanol group. This may indicate that the second intranasal administration of butorphanol in patients randomized to butorphanol prevented a further rise in pain despite the fact that shocks with increasing intensicy were delivered.

Comparison of Placebo Group With Butorphanol Group for Patients Who Received the Same Shock Intensity Ending Stage I and Going to Stage II

Eighteen of the 23 patients randomized to placebo and 17 of the 24 parients randomized to butorphanol requested (additional) butorphanol and were again defibrillated by use of the previous shock intensity before continuation of the step-up protocol. In the patients randomized to placebo, the mean VAS score for 
Table 4.-. Mean stope of pain and fear variables against shock number during stages I and II according to trearment.

\begin{tabular}{|c|c|c|c|c|c|c|}
\hline \multirow[b]{2}{*}{$\begin{array}{l}\text { Pain and fear } \\
\text { variable }\end{array}$} & \multicolumn{3}{|l|}{ Stage I } & \multicolumn{3}{|l|}{ Stage II } \\
\hline & $\begin{array}{l}\text { Placebo } \\
(n=23)\end{array}$ & $\begin{array}{l}\text { Butorphanol } \\
(n=24)\end{array}$ & P & $\begin{array}{l}\text { Placebo } \\
(\mathrm{n}=18)\end{array}$ & $\begin{array}{l}\text { Butorphanol } \\
(n=17)\end{array}$ & P \\
\hline VAS-H & $8.4+2.1 *$ & $5.6 \pm 2.0^{*}$ & 0.24 & $10.0 \pm 3.3^{*}$ & $6.3 \pm 2.2^{*}$ & 0.34 \\
\hline VASP & $18.0 \pm 4.6^{*}$ & $14.0 \pm 3.3^{*}$ & 0.74 & $14.0 \pm 4.2^{*}$ & $4.5 \pm 2.4$ & 0.057 \\
\hline PRL-T & $5.7 \pm 1.2$ & $3.6 \pm 1.2^{*}$ & 0.093 & $4.4 \pm 1.1 \%$ & $1.0 \pm 0.95$ & 0.0099 \\
\hline PRI-S & $3.1 \pm 0.74^{4}$ & $1.9 \pm 0.77^{*}$ & 0.074 & $2.1 \pm 0.50^{*}$ & $0.46 \pm 0.50$ & 0.019 \\
\hline PRU-A & $1.3 \pm 0.28^{4}$ & $0.85 \pm 0.45$ & 0.062 & $0.98 \div 0.36^{*}$ & $0.28 \pm 0.33$ & 0.44 \\
\hline PRIE & $1.3 \pm 0.29^{*}$ & $0.85 \pm 0.23^{*}$ & 0.23 & $1.4 \pm 0.31^{\text {*i }}$ & $0.30 \pm 0.29$ & 0.015 \\
\hline
\end{tabular}

A: affective; E: evaluative; F: fear; n: number of patients; $P$ : pain; PRI: pain rating index; $S$ : sensory; T: rotal; VAS: visual analogue scale. Values are mean $\#$ SEM unless orherwise specified. "Slopes are significantly different from $0(\mathrm{P}<0.05)$.

pain, PRI-T, PRI-S and PRI-E became significantly lower after butorphanol administration, whereas no significant difference was found for the mean VAS score for fear and PRI-A. In the patients randomized to butorphanol, no significant difference was found for the pain and fear variables after a second butorphanol administration (Table 5).

\section{INTRAVENDUS SEDATION (STAGE III)}

No side effects from butorphanol administration were observed. There was no significant difference in the number of patients of the placebo and butorphanol group who required intravenous sedation for disconfort relief to obtain a successful cardioversion (13 patients of the placebo group and 10 of the butorphanol group; $\mathbb{P}$ $=0.47)$. Intravenous etomidate was given to 12 patients of the placebo group and to 7 of the butorphanol group; the mean dose was not significantly different for both groups $(15.0 \pm 5.8$ vs $15.4 \pm 5.9 \mathrm{mg} ; \mathrm{P}=0.89)$. One patient in the placebo group received $12 \mathrm{mg}$ midazolam intravenously and 3 patients in the butorphanol group received a mean dose of $7.5 \pm 1.8 \mathrm{mg}$. The mean amount of energy at which patients requested intravenous sedation also did not differ between the placebo and the butorphanol group $(3.1 \pm 1.4 \mathrm{vs} 2.7 \pm 1.6 \mathrm{~J} ; \mathrm{P}=0.58)$. The corresponding values in voltage for both patient groups were $221 \pm 54$ and $205 \pm 62 \mathrm{~V}(\mathrm{P}=0.52)$. 
Table 5. - Comparison of pain and fear variables for patients of the placebo and butorphanol groups who received the same shock incensicy ending stage l and going ro stage II.

\begin{tabular}{|c|c|c|c|c|c|c|}
\hline \multirow[b]{2}{*}{ Pain and fear variable } & \multicolumn{3}{|c|}{ Placebo $(\mathrm{n}=18)$} & \multicolumn{3}{|c|}{ Butorphanol $(n-17)$} \\
\hline & Stage 1 & Stage II & $\mathrm{P}$ & Stage & Stage II & $P$ \\
\hline WAS-E & $50 \div 31$ & $45+36$ & 0.311 & $39 \pm 27$ & $41 \pm 32$ & 0.39 \\
\hline VAS-B & $52 \pm 34$ & $36 \pm 31$ & 0.023 & $40 \pm 32$ & $37 \pm 30$ & 0.89 \\
\hline PRI-T & $27 \pm 10$ & $23 \pm 11$ & 0.029 & $24 \pm 12$ & $25 \pm 13$ & 0.39 \\
\hline PRI-S & $14 \pm 7.2$ & $12 \pm 7.5$ & 0.030 & $13 \pm 7.8$ & $14 \pm 7.7$ & 0.31 \\
\hline PRI-A & $5.4 \pm 2.6$ & $5.3 \pm 2.5$ & 0.55 & $4.3=4.0$ & $4.9 \pm 3.6$ & 0.18 \\
\hline PRI-E & $7.1 \pm 2.0$ & $5.8 \pm 2.1$ & 0.023 & $6.9 \pm 2.3$ & $6.3 \pm 2.6$ & 0.22 \\
\hline
\end{tabular}

Abbreviations as in Table 4 . Values are mean $\pm S D$.

\section{DISCUSSION}

Shock-related discomfort may be due to pain and/or fear and may result from several factors such as skeletal muscle contraction, nerve scimulation and psychological components. 1,2 To date, most studies have attempted to cause less pain by lowering the atrial defibrillation threshold by use of technological improvements of the defibrillation system, ${ }^{13,14}$ shock characteristics, $3,14,15$ and optimal electrode localization ${ }^{4,16}$ or by administration of antiarrhythmic drugs $s^{17}$. To our knowledge, the current study is the first to evaluate in a double-blind placebo-controlled manner the effect of an analgesic on pain and fear during internal atrial defibrillation. All patients of this study petceived pain and fear during internal cardioversion, and the value of both discomfort parameters increased after serial delivery of shocks with increasing intensity. As expecred, fear was not affected by the opioid, but burorphanol diminished pain perception during internal defibrillation. The dose of butorphanol needed to obtain this effect depends on the time of analysis. By analysis of the effect of butorphanol on pain over time, the study reveals a trend towards an effect of $1 \mathrm{mg}$ butorphanol. A total of $2 \mathrm{mg}$ of the pain-relieving drug prevented a further rise in pain despite the delivery of shocks with increasing intensity. By analysis of the effect of butorphanol on pain at well-defined moments of the study, 2 observations were made. First, after the first shock there was no difference in pain perception between the placebo and butorphanol groups; however, when patients received the same shock intensiry ending stage I and going to stage II, administration of butorphanol clearly decreased pain perception in the former placebo group. This confirms $1-3,5,13$ that the delivered voltage and/or energy play a role in pain perception. Ar the beginning of the step-up protocol, a low-energy shock did not result in pain perception. In contrast, at the moment when the patients requested additional medication for discomfort and a higher amount of energy was delivered in comparison to the beginning of the study, pain perception clearly decreased in the placebo group when butorphanol was given. Second, at this partial cross-over point of the 
study, a redose of the analgesic did not further diminish pain in the burorphanol group. One can hypothesize that butorphanol reduces pain perception to a certain level that can not be lowered further despite redose of the drug.

Of the 3 qualitative components of pain, only the affective component was not influenced by butorphanol at the partial cross-over point nor over time. This finding is in agreement with the lack of an effect of an analgesic on fear because the affective dimension of pain is related to emotional qualities of pain perception such as stress, fear and autonomic reactions. A further increase of the sensorial component, indicaring physical pain characteristics such as pressure and temperature, and the evaluative component of pain, reflecting a cognitive measurement of pain severity, was prevented by the second dose of the analgesic despite the fact that shocks with increasing intensity were delivered.

Although previous work used the VAS ${ }^{4}$ or a similar verbal scale $e^{1-3,5,13}$ to evaluate pain during the course of a step-up atrial defibrillation protocol, this study showed that whe total PRI of the 3 analyzed pain dimensions appear to be more accurate than the VAS. In a comparison of both patient groups during stage I of the study, the PRI-T revealed a trend towards an effect of $1 \mathrm{mg}$ of butorphanol. Furthermore, a significant lower PRI-T during stage II of the study protocol undoubtedly demonstrares that the second dose of the pain-relieving drug prevents a further rise in pain despite the delivery of higher-intensity shocks. In contrast, the VAS did not show a difference between placebo and butorphanol on pain relief during stage I and showed only a trend towards an effect on pain perception after $2 \mathrm{mg}$ of the opioid. It is known that there exists only a moderate correlation between the VAS and MPQ despite the very different patient groups studied. ${ }^{10,18}$ In addition, VAS measures are known to be more difficult to use and understand in some parients. ${ }^{19}$

Recently, the safery and efficacy of an implantable atrial defibrillator (Metrix Atrioverter) was evaluated in 51 patients with recurrent atrial fibrillarion. ${ }^{20}$ The mean atrial defibrillation threshold of the currently available model 3020 was $2.9 \pm$ 1.1 I at implantation and was slightly lower 3 months thereafter. In our study, approximately the same mean amount of energy $(3.1 \pm 1.5 \mathrm{~J}$ for the placebo group and $2.7 \pm 1.6 \mathrm{~J}$ for the butorphanol groupl was tolerated by $49 \%$ of the parients before they required intravenous sedation. The other $51 \%$ of the patients were successfully cardioverted. without the need for intravenous sedation, at a mean energy of $2.3 \pm 1.5 \mathrm{~J}$. The favorable effect of butorphanol, ease in intranasal administration, absence of side effects and ability to alleviate pain at the required amount of energy needed for successful Atrioverter therapy makes the drug suitable for ambulatory use with the Arrioverter. Nevertheless, our data show that psychological components causing anxicty substantially contribute to discomfort during the delivery of sequential shocks. Until now, shock-related discomfort is only evaluated in the catheterization laboratory. Because an Atrioverter is inrended for ambulatory use, it may also be important to consider the effect of surrounding environmental stimuli and patient posture on shock tolerability. The somewhat distracting effects of the 
surrounding stimuli may alrer the patient's perception of the shocks. Because posture may affect the relative location of the electrical field with respect to nerves and muscles, shocks delivered in a supine position may be perceived differently than shocks delivered in an upright position. Additionally, previous studies showed that tolerability of internal atrial cardioversion by use of a step-up protocol was more related to the number of shocks delivered than to the shock intensity. 4,5 This suggests thar patients with an Atrioverter may better tolerare the delivery of 1 or a few higher-intensity shocks rather than a greater number of lower-intensity shocks.

\section{CONCLUSIONS}

This study, using a step-up internal atrial defibrillation protocol, demonstrates an effect of an opioid analgesic, butorphanol, on pain perception during internal atrial defibrillation. Of the 3 qualitative components of pain, only the affective component was not influenced by butorphanol. The PRI-T evaluated pain more accurately than the VAS score. If, in patients treated with an Atrioverter, shock-related discomfort is mainly due to pain, transnasal butorphanol would provide a merhod to relief pain in a fast and convenient manner in the outpatient setting. Nevertheless, this study also shows that psychological components causing anxiety substantially contribute to discomfort during defibrillation. Control of the anxiety component of discomfort will require further clinical evaluation.

\section{REFERENCES}

1. Murgatroyd FD, Slade AKB, Sopher SM, Rowland Ex, Ward DE, Camm AJ. Efficacy and rolerability of transwenous low energy cardioversion of paroxysmal atrial fibrillation in humans./ Am Coll Cardiol 1995;25:1347-1353.

2. Lévy S, Ricard P, Gueunoun M, Yapo F, Trigano J, Mansouri C, Paganelli F. Low-nargy cardioversion of spontaneous atrial fibrillation: immediate and long-tem results. Circulation. $1997 ; 96: 253-259$.

3. Ammer R, Alt E, Ayers $G$, Schmirt C, Pasquantonio J. Schmidr M, Pátrer K, Schömig A. Pan threshold for low energy intracardiac cardioversion of atrial fibrillation with low or no sedation. Pacing Clin Electroplysiol. 1997;20:230-236.

4. Lok NS, Lau CP, Tse HF, Ayers GM. Clinical shock tolerability and ffect of different vighte anrial electrode locations on efficacy of low energy human transyenous atrial defibrillation using an implantable lead syscem. I Am Coll Cardiol 1997:30:1324-1330.

5. Boriani G, Biffi M, Bronzetti G. Ayers GM, Zannoli R, Branzi A. Capucci A. Magnani B. Efficacy and tolerability in fully conscious patients of transwenous lowenergy internal arrial cardiowersion for atrial fibrillation. Ans / Catdiol. 1998;81:241-244. 
6. Thmencmanc C, Rodriguex LM, Stheets LLM, Wellens HJ. Inmediate reinitiation of atrial fibrillation following internal atrial defibrillation. J Cardiotrasc Electrophysiol. 1998;9:122-127.

7. Gillis JC, Benfield I*, Goa KL. Transnasal butorphanol. A review of its pharmacodynamic and pharmacokinetic properties, and therapeutic potential in acure pain management. Dwogs. $1995: 50(1): 157-175$.

8. Verkes RJ, Vanderier K, Vertommen H, van der Kloot WA, van der Meij J. De MPQ-DLV, cen standaard Nederlandstalige versie van de McGill Pain Questionnaire voor Belgie en Nederland. In: wan der Koot WA, Verrommen H, eds. De.MPQ-DLV, en statudard Nederlandstalige versie wan de McGull Pain Questionnaire: achtergranden en handleiding. Lisse: Swets \& Zeitlinger, 1989;57-69.

9. Melwack R. The MeGill Pain Questionnaire: major properties and scoring methods. Pain. $1,975: 1: 277-299$.

10. Vlaeyen I.W.S. Chronic low back paln. Assessment and treatment from a behawioral rehabilitation perspective. (Thesis, University of Maastrichr). Lisse/Rockland: Swets \& Zeitlinger; 1991.

11. Ayers GM, Alferness CA, Iina M, Wagner DO, Sirokman WA, Adams JM, Griffin JC. Ventricular proarhythmic effecrs of ventricular cycle length and shock strength in a sheep model of transwenous anrial defibrillation. Circulation. 1994;89:413-422.

12. Timmermans C, Rodriguez LM, Smeets 『LRM, Ripley K, Ayers GM, Wellens HJJ. Effecr of intranasal butorphanol on discomfort during internal atrial defibrillation. I Am Coll Cardiol. 1997:29,2(suppl A):474A. Abstract

13. Tomassoni $G$, Newby KH, Kearney MM, Brandon My, Barold H, Natale A. Testing different biphasic waveforms and capacirances: effect on atrial defibrillation threshold and pain perception. $J$ Am Coll Cardiol. 1996:28:695-699.

14. Cooper RAS, Plumb VJ, Epsrein AE, Kay GN, Ideker RE. Marked reduction in internal atrial defibrillation thresholds with dual-current pathways and sequential shocks in humans. Cinculation. 1998:97:2527-2535.

15. Cooper RAS, Johnson EE, Wharron JM. Internal atrial defibrillation in humansi improved efficacy

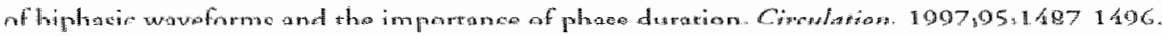

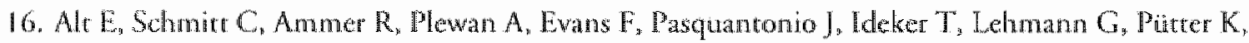
Schomig A. Effect of electrode position on outcome of low-energy intracardiac cardiowersion of atrial Gilurilarion. Am / Cardiol 1997,79:621-625.

17. Lau CP. Lok NS. A comparison of ranswenous atrial defibrillation of acute and chronic atrial fibrillation and the effec of incravenous sotalol on human atral defibrillation threshold. Pacing Clin Electrophysiol. 1997:20:2442-2452.

18. Melzack $\mathrm{R}$, Katz J. The McGill Pain Questionnaire: appraisal and current status. In: Turk DC, Melzack $\mathbb{R}$, eds. Hamdbok of patim assesment. New York, NY: Guilford Press; 1992:152-168.

19. Jensen MP, Karoly P, ORiordan EF, Bland F, Burns RS. The subjective experience of acute pain: an assessment of the utrility of 10 indices. Chin J Pain 1989:5:153-159.

20. Wellens HJI. Lau CP, Lüderiz B, Akhtar M, Waldo AL, Camm AJ, Tümermans C, Tse HF, Jung W, Jordaens L, Ayers $\mathrm{G}$, for the Metrix Investigarors. Arrioverter: an implantable device for the treatment of atrial fibrillation. Cinculation. 1998;98:1651-1656. 
CHATER 5

\section{Immediate reinitiation of atrial fibrillation following internal atrial defibrillation}

Carl Timmermans, MD; Luz-Maria Rodriguez, MD; Joep LRM Smeets, MD; Hein If Wellens, MD

From the Department of Cardiology, Academic Mospiral Maastrichr, Maastrich, The Netherlands

Abstract presented at the $70^{\text {th }}$ Anwual Scientific Session of the American Heatr Assaciation 1997 (Circulution 1997,96:1-1150)

Pablished in J Cardiowas Electropbysiol 1998:9:122-128 


\section{A B STRACT}

Introduction. Although the recurrence rate of atrial fibrillation has been reported to be similar to that after external and internal cardioversion, little is known about immediate reinitiation of atrial fibrillation (IRAF) following internal cardioversion. Methods and Results. Thirty-eight patients (24 men; mean age $63 \pm 13$ years) underwent internal atrial defibrillation. Catheter-based defibrillation electrodes were positioned in the anterolateral right atrium and the coronary sinus. All patients were cardioverted at a mean threshold of $4.6 \pm 3.4 \mathrm{~J}$. Five of 38 patients (13\%) had 1 to 4 episodes of IRAF. No difference in clinical and echocardiographic characteristics were observed when patients with and without IRAF were compared. Atrial fibrillarion was always reinitiated by an atrial premature beat. When the earliest atrial endocardial activation time on the defibrillation catheters was analyzed, these atrial premature beats did not seem to originate from the defibrillation carheters. Twenty-one patients had atrial premarure beats without IRAF. When the coupling intervals of the first atrial premature beat in patients without and with IRAF after conversion were compared, a significant difference was found (661 \pm 229 vs $418 \pm$ 79 msec, $P<0.05)$. IRAF was successfully treated with repeated shock delivery after the administration of atropine in 1 patient and intravenous flecainide in 2. Only repeated shock delivery was sufficient to treat IRAF in another 2 patients. Late recurrences of atrial fibrillation occurred in 3 of 5 with IRAF and in 19 of 33 patients wirhout IRAF ( $\mathrm{P}=\mathrm{NS})$.

Conchsions. IRAF after intemal atrial defibrillation occurred in $13 \%$ of parients, was always initiated by an arrial premature beat having a short coupling interval not originating from the defibrillation catheters, and was prevenred by repeated shock delivery with or without preceding administration of pharmacologic agents. IRAF did not predict early recurrences of the arthythmia after discharge from hospital, emplasizing the necessity to treat immediate reinitiation promptly to achieve a successful cardioversion. 
External and intra-atrial shocks are given in an attempt to convert atrial fibrillation to sinus thythm with a higher acute success rate of intra-atrial shocks but similar long-term recurrence rates of atrial fibrillation '. Little is known about the immediate reinitiation of atrial fibrillation (IRAF) following the use of either one of these techniques $^{2-5}$. Immediate reinitiation may be one of the factors derermining an unsuccessful electrical cardioversion and may have important implications for patients with an implantable atrial defibrillaror. The aim of this study was to report our observations on this intriguing phenomenon of IRAF in a cohort of consecutive patients who underwent internal arrial defibrillation.

\section{METHODS}

\section{STUDY POPULATION}

Thirty-eight consecutive patients with atrial fibrillation ( 24 men and 14 women; mean age $63 \pm 13$ years, range 28 to 85 ) in whom conversion to sinus rhythm was clinically indicated underwent internal atrial defibrillation. Evaluation of the patients included a clinical history, physical examination, routine laboratory and thyroid function tests, 12-lead ECG, and transthoracic and transesophageal echocardiography. Parients were excluded from the study if they had any one of the following criteria: (1) an implanted artificial heart valve; (2) a previous history of a sustained ventricular arrhythmia, cardiac arrest, or congenital long QT syndrome; (3) atrial fibrillation due to reversible causes (e.g., hyperthyroidism); (4) a history of myocardial infarction or a revascularisation procedure within the past 6 months; (5) a history of thromboembolic events; (6) a left atrial thrombus diagnosed by transesophageal. echocardiography obtained the day before cardioversion; (7) digitalis roxicity; (8) significant electrolyte imbalance; (9) nor been anticoagulared for at least 4 weeks; or (10) an implanted pacing device. The administration of antiarrhythmic drugs was not interrupted for the procedure. Anticoagulation was interrupted 2 days before the procedure. "The study protocol was approved by the Medical Ethics Committee of our Hospital, and written informed consent was obtained from all patients.

\section{INTERNAL CARDIOVERSION PROTOCOL}

Two temporary 6-French catheters (Elecath, Electro-Catheter Corp., Rahway, NJ, USA) with an active surface area of $2.62 \mathrm{~cm}^{2}$ consisting of 10 parallel stainless steel rings with a $5-\mathrm{mm}$ ellectrode size and a $2-\mathrm{mm}$ interelectrode distance were inserted into the right femoral vein and positioned in the anterolateral right atrium and coromary sinus (Fig. 1). In one parient with congenital heart disease, the coronary sinus could not be cannulated, and the catheter was positioned in the left atrium through an atrial septal defect. Using the right atrial catheter as the cathode and the coronary 
$52 \mid$ Chappers

A

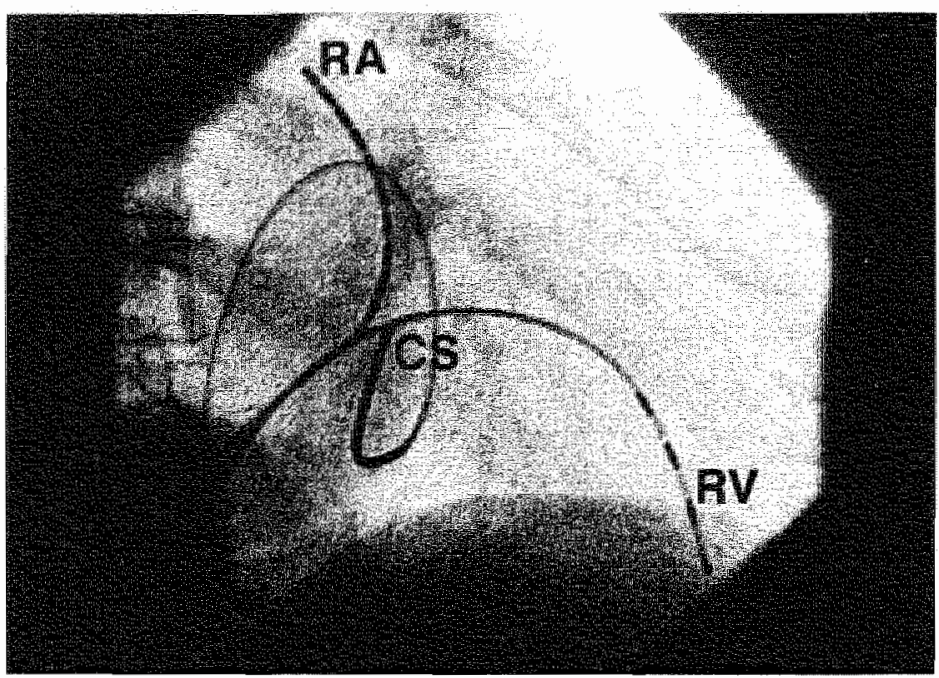

B

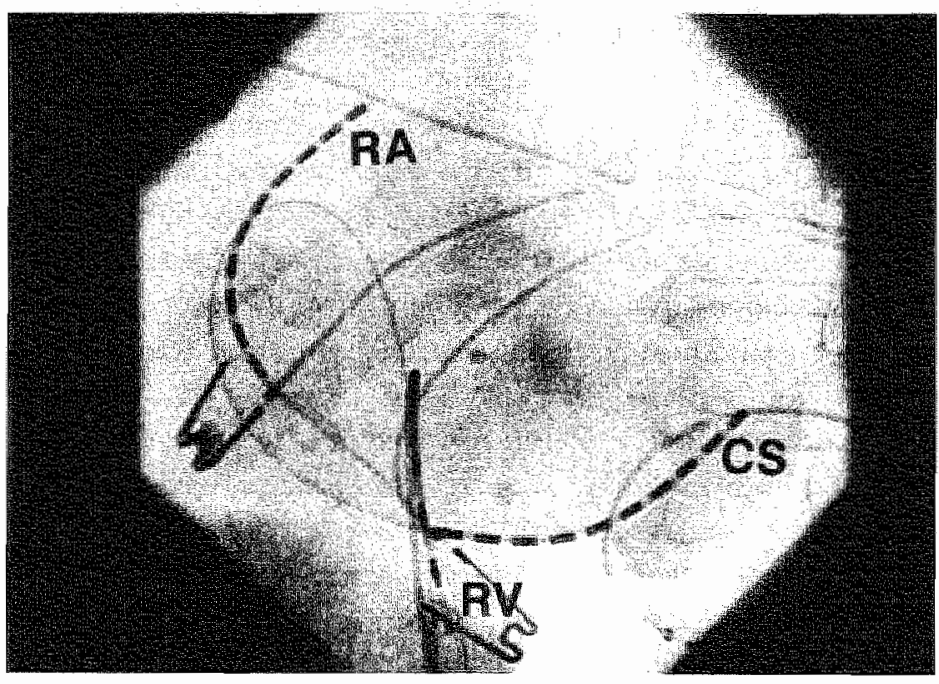

Figure 1. - Hight (pand A) and left (panel B) oblique fluoroscopic projecrions of the posinon of the defibrillation electrodes in the anterolateral right atrium (RA) and the coronary sints (CS). A guadripolar catheres is positioned at the right ventricular apex (RW) to allow postshock temporary ventricular pacing. 


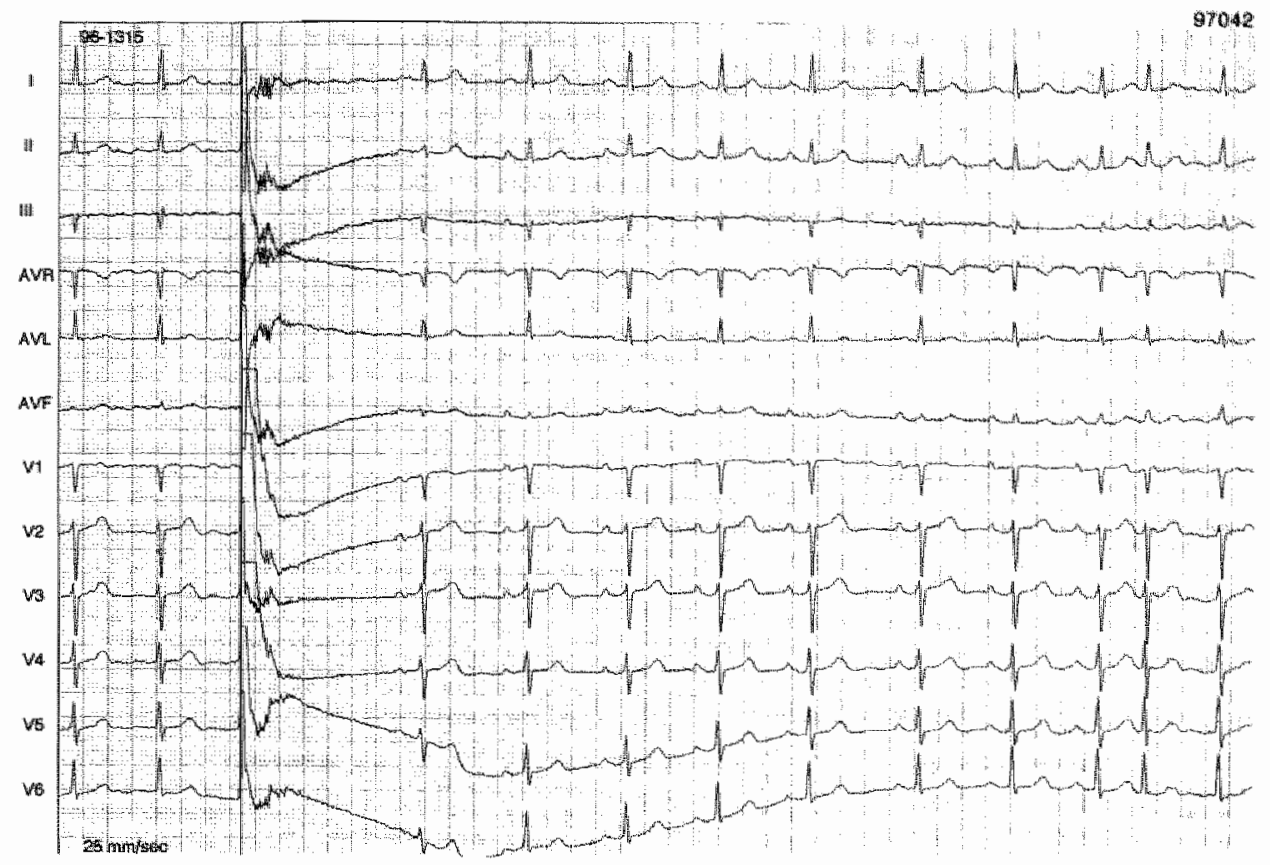

Figure 2. - Twelve-lead ECG recorded during internal arrial defibrillation. A I-j biphasic shock, synchronized to the $\mathrm{R}$ wave, rerminated arrial fibrillation. Note that after 8 sinus bears, an atrial premarure bear reinitiated atrial fibrillation.

sinus catheter as the anode, a $6 / 6$ msec defibrillation shock, synchronized to the $\mathrm{R}$ wave, was delivered from an external defibrillator (Ventitrex HVS-02 [Ventitrex, Sunnyvale, CA, USA]). To prevent inadvertent induction of ventricular fibrillation, shocks were delivered only after RR intervals more than $500 \mathrm{msec}^{6}$. A quadripolap catherer (USCI, Billerica, MA, USA) was positioned at the right ventricular apex to allow temporary ventricular postshock pacing. Shocks were delivered using all 10 electrodes of both defibrillation catheters, starting at $90 \mathrm{~V}$, following a $40-\mathrm{V}$ step-up protocol until cardioversion of atrial fibrillarion occurred. A custom-made switch box enabled immediate recording of the endocardial bipolar electrograms from 8 of the 10 electrodes of both defibrillation catheters after shock delivery. The time interval berween shocks was 5 minutes. During the study, the 12-lead ECG and endocardial electrograms were recorded and stored by PC-EMS 3.0 (Cardiovascular Research Institute, Maastricht, The Netherlands). Afrer successful internal atrial defibrillation, extracardiac and intracardial electro(cardio)grams were analyzed at paper speeds of 25 and $100 \mathrm{~mm} / \mathrm{sec}$ to study IRAF. In case of occurrence of atrial premature beats without and with IRAF, the following parameters of the first atrial premature beat were analyzed: the relation between the onset of the P wave on the 12-lead ECG and the earliest atrial endocardial activation time on the defibrillation catheters; and the coupling interval of the atrial premature beat and the PP interval 
preceding the arrial premature beat. The following treatment strategy was used to treat IRAF. Fist a repeated defibrillation shock was given. If atrial fibrillation reoccurred, another internal cardioversion was performed after the administration of pharmacologic agents. Because of its short-lasting parasympathicolytic effect, $1 \mathrm{mg}$ atropine was first given. In case of a third recurtence of atrial fibrillation. $1.5 \mathrm{mg} / \mathrm{kg}$ intravenous flecainide was given.

\section{DEFINITION}

IRAF was defined as the resumption of atrial fibrillacion within 1 minure after an internal atrial defibrillation shock that resulted in sinus thythm for at least one beat. An example of IRAF is shown in Figure 2.

\section{FOLLOW-UP}

All patients had 24-hour Holter monitoring immediately after successful atrial defibrillation. Every patient was seen in the outpatient clinic 6 weeks after internal defibrillation and thereafter at 1 -month intervals.

\section{STATISTICAL ANALYSIS}

Continuous variables are expressed as mean values $\pm \mathrm{SD}$ and compared between groups using the Student t-test. Nominal values were evaluated by the Chi-square test. $P<0.05$ was considered statistically significant.

\section{RESULTS}

\section{CLINICAL CHARACTERISTICS}

The studicd population was divided according to the absence or the presence of IRAF afrer a successful defibrillation shock. Table 1 shows the clinical and echocardiographic characteristics of the two patient groups. No significant difference was found between groups according to gender, age, duration of atrial fibrillacion as disease, duration of the treated atrial fibrillation episode, presence of structural heart disease, use of antiarthythmic drugs, left atrial diameter, and left ventricular ejection fraction. The majority of the patients in both groups were taking Class III anriarthythmic drugs (20 patients without IRAF and 3 with IRAF; P $>0.05$ ). 
Table 1. - Clirical and echocardiographic characteristics of patients witl and without IRAl following internal atrial defibrillarion.

\begin{tabular}{|c|c|c|}
\hline & $\begin{array}{l}\text { No IRAF } \\
(\mathrm{n}=33)\end{array}$ & $\begin{array}{l}\text { IRAF } \\
(n=5)\end{array}$ \\
\hline Men/women & 20113 & $4 / 1$ \\
\hline \multicolumn{3}{|l|}{ Age $(y r)$} \\
\hline Maan $\pm S D$ & $63 \pm 13$ & $60 \pm 12$ \\
\hline Range & $28-85$ & $47-76$ \\
\hline \multicolumn{3}{|l|}{ Durarion $A F$ as disease $(y r)$} \\
\hline Mean $\pm S D$ & $6.8 \pm 5.8$ & $5.0 \pm 3.9$ \\
\hline Rangé & $0.08-20$ & $1.13-10$ \\
\hline \multicolumn{3}{|l|}{ Duration treated AF episode (days) } \\
\hline Mean \pm SD & $211 \pm 404$ & $318 \pm 472$ \\
\hline Range & $1-2100$ & $1-1000$ \\
\hline \multicolumn{3}{|l|}{ Seructural heart disease } \\
\hline Ischemic & 7 & 1 \\
\hline Valvular & 4 & 0 \\
\hline Cardiomyopathy & 3 & 0 \\
\hline Hypertensive & 11 & 2 \\
\hline Congenital & 1 & 0 \\
\hline Lone AF & 7 & 2 \\
\hline \multicolumn{3}{|l|}{ Current antiarthythmic drug } \\
\hline Class I & 0 & 0 \\
\hline Class II & 2 & 0 \\
\hline Class III & 12 & 1 \\
\hline Class $\mathrm{V}$ & 1 & 2 \\
\hline Digitalis & 7 & 0 \\
\hline Combination of antiartythmic drugs & 9 & 2 \\
\hline No antiarnythmic drug & 2 & 0 \\
\hline \multicolumn{3}{|l|}{ LA dianeter (mm) } \\
\hline Mean $\pm 5 D$ & $53 \pm 6$ & $49 \pm 5$ \\
\hline Range & $42-70$ & $42-55$ \\
\hline \multicolumn{3}{|l|}{$\mathbb{L V E F} \%$} \\
\hline Mean $\pm S D$ & $55 \pm 13$ & $60 \pm 8$ \\
\hline Range & $20-73$ & $48-68$ \\
\hline
\end{tabular}

Data presented are number of parients unless otherwise specified. AF: atrial fibrillation; IRAF: inmediate reiniriation of atrial fibrillarion; LA: lefr arial; LVEF; left wentricular ejection fraction. P value nor significant. 
Table 2. - Mode of initianion of IRAF and neatment of that episede.

\begin{tabular}{|c|c|c|c|c|c|c|c|c|c|c|c|}
\hline Patient No. & 1 & & 2 & & & & 3 & & & & 5 \\
\hline Shock intensity (1) & 1 & 2 & 0.5 & 1 & 1 & 1 & 6 & 6 & 6 & 4 & 12 \\
\hline Time to IRAF (sec) & 7 & 18 & 2.5 & $32^{*}$ & 30 & 55 & 5 & 6 & 3 & 2 & 15 \\
\hline $\mathrm{APB}$ coupling interval (msec) & 310 & 320 & 480 & 410 & 390 & 390 & 400 & 440 & 580 & 400 & 380 \\
\hline $\begin{array}{l}\text { PP interval preceding APB } \\
\text { (msec) }\end{array}$ & 700 & 710 & 960 & 810 & 660 & 570 & 800 & 840 & 980 & NA & 640 \\
\hline \multicolumn{12}{|l|}{ Therapy } \\
\hline unsuccessful & HI & & $\mathrm{HI}$ & & A & & $\$$ & A & & & \\
\hline stuccessful & & $A$ & & & & $\mathbb{F}$ & & & F & $\mathrm{HI}$ & $\mathrm{HI}$ \\
\hline
\end{tabular}

*Spontaneous termination and reinitiation of atral fibrillation; A: acropine; APB: atrial premature bear; $F$ : Alecainide; HI: higher inrensity defibtillation shock, IRAF: immediate reiniriation of atrial fibrillation; NA: not atkallable; SI: same intensity defibrillation shock.

\section{ACUTE RESULTS OF INTERNAL ATRIAL DEFIBRILLATION}

All parients were successfully cardioverted at a mean threshold of $4.6 \pm 3.4$ (range 1 to 15) J without complications. No significant difference was found when patients withour and with IRAF were compared with regard to their mean defibrillation threshold ( $4.5 \pm 3.0$ vs $5.6 \pm 5.7 \mathrm{~J} ; \mathrm{P}>0.05)$.

\section{PATIENTS WITH IRAF}

Five of 38 patients (13\%) had 1 to 4 episodes of IRAF (Table 2). IRAF occurred after defibrillation shocks ranging from 0.5 to $12 \mathrm{~J}$. The time interval between the termination of atrial fibrillation and reinitiation varied from 2 to 55 seconds. The earliest atrial endocardial activation time recorded from the defibrillation catherers was always after the beginning of the $\mathrm{P}$ wave of the initiating atrial premature beat (Fig. 3). Based on this observation, these atrial premature beats did nor seem to originate from the defibrillation catheters. The coupling interval of the atrial premarure beat initiating atrial fibrillation after a successful defibrillation shock ranged from 310 to $580(418 \pm 79) \mathrm{msec}$. The PP interval preceding the atrial premature beat reinitiating atrial fibrillation varied from 570 to $980(767 \pm 135)$ msec in 10 available episodes. In patient 4, this PP interval could not be measured because atrial fibrillation restarted immediately after the first sinus depolarization following defibrillation. In patients 4 and 5, episodes of immediate recurrences were treated successfully by increasing the shock intensity. In parient 1, a higher shock intensity in combination with atropine ( $1 \mathrm{mg}$ ) succeeded in preventing IRAF. In patient 2 , a higher shock intensity did not prevent the occurrence of IRAF. The latter episode terminated spontaneously after 32 seconds, reinitiating again after 10 seconds. Atropine ( $1 \mathrm{mg}$ ) with a defibrillation shock intensity of $1 \mathrm{~J}$ did not prevent IRAF. This episode was successfully converted with $1 \mathrm{~J}$ after administration of 100 -mg intrave- 


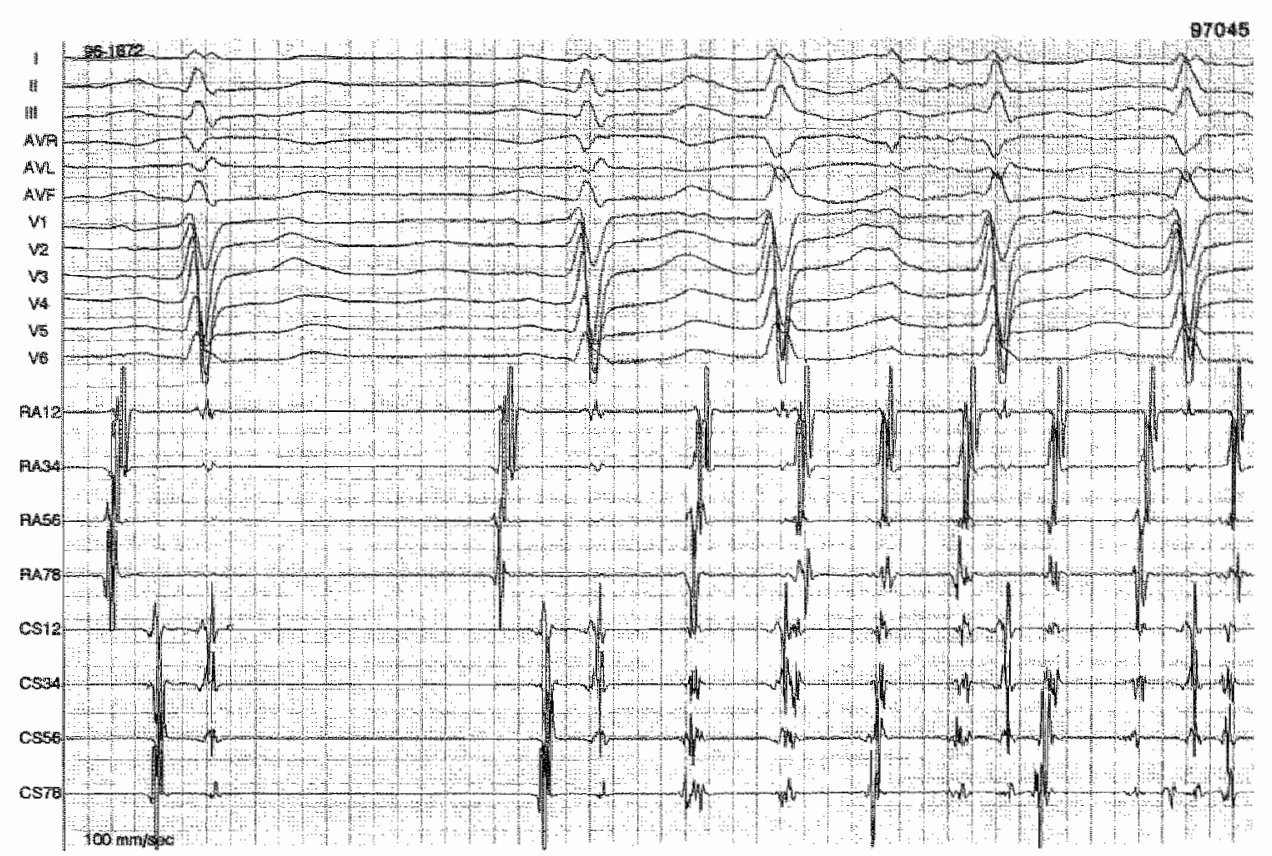

Figure 3. - Twelve-lead ECG shown with the endocardial recordings of the anterolateral right atrial and coronary sinus defibrillation electrodes. The bipolar electrograms of each defibrillation electrode ne numbered from distal to proximal. After 2 sinus beats, an atrial premature beat with earliest endocardial activation in the coronary sinus bipolar electrogram CS78 reinitiates atrial filorillation. Nore that the beginning of the arrial premarure beat on the 12-lead ECG occurs before the earliest atrial endocardial activation on the coronary sinus defibrillation catherer.

nous flecainide intravenously. A 6-J defibrillation shock did not prevent the IRAF in patient 3, nor did the administration of $1 \mathrm{mg}$ of atropine before defibrillating with the same shock intensity. Prevention of IRAF was obtained by defibrillating with 7 J after administration of 150 -mg intravenous flecainide.

Twenty-one patients had atrial premature beats without IRAF during the first minute after a successful defibrillation shock. Their clinical and echocardiographic characteristics did not differ from those of the patients with IRAF. The earliest atrial endocardial activation time recorded from the defibrillation catheters was always after the beginning of the $\mathbb{P}$ wave of the first atrial premature beat. When the coupling intervals of the first atrial premature beat in patients with atrial premature beats without and with IRAF after conversion were compared, a significant difference was found $(661 \pm 229$ vs $418 \pm 79 \mathrm{msec}, \mathbb{P}<0.05)$. The $\mathbb{P P}$ interval preceding the atrial premature beat could only be studied in 10 of 24 parients with atrial premarure beats without IRAF and was compared to that of patients with IRAF. Although, the PP interval was longer in patients without IRAF than in those with IRAF, the difference was not statistically significant (1036 478 vs $767 \pm 135 \mathrm{msec} ; P>0.05)$. 
There were three recurrences of atrial fibrillation in the five patients with IRAF during a mean follow-up of $162 \pm 36$ days. The mean interval of recurrence of atrial fibrillation was $4.1 \pm 3.9$ days (median 4.0 , range 0.2 to 8 ). In the 33 patients without IRAF, the arrhythmia recurred in 19 patients during a mean follow-up of $114 \pm 55$ days. The mean interval of recurrence of atrial fibrillation was $24.5 \pm 49.8$ days (median 5.5, range 0 to 173 ). The recurrence rate, the time interval of recurrence of atrial fibrillation, and the duration of follow-up were not significantly different between both groups $(\mathrm{P}>0.05)$.

\section{DISCUSSION}

IRAF can occur after external and internal atrial defibrillation. ${ }^{2-5}$ The incidence of IRAF after external defibrillation has nor been studied systematically. After internal atrial defibrillation, some studies ${ }^{3,4}$ have reported an incidence of $8 \%$. In the present study, IRAF occurred in 5 of 38 consecutive patients (13\%). The reasons for early recurrences of atrial fibrillation after electrical cardioversion are not entirely clear. It has been suggested from clinical. studies that factors such as the duration of atrial fibrillation, atrial size, and underlying heart disease may play a role in reappearance of atrial fibrillation. In the study population, clinical characteristics such as gender, age, duration of atrial fibrillation, duration of the treated episode, structural heart disease, current antiarrhythmic drugs, and echocardiographic data such as left atrial diameter and left ventricular ejection fraction did not influence the occurrence of IRAF. In our study, IRAF was always initiated by an atrial premature beat. Further analyzing the episodes of IRAF with regard to ECG and defibrillation parameters, the only variable that influenced the occurrence of IRAF was the coupling interval of the iniriating atrial premature beat. This coupling interval was shorter than the coupling interval of the atrial premature beats not initiating atrial fibrillation ( $418 \pm$ 79 vs $661 \pm 229 \mathrm{msec}$ ). The PP interval preceding the atrial premature beat did not seem to play a role in the reinitiarion of atrial fibrillation. These findings are in agreement with the observations of Capucci et al. ${ }^{7}$ In their study, patients with lone atrial fibrillation were evaluated by Holter recording, and the coupling interval of the atrial premarure beats initiating spontaneous episodes of atrial fibrillation was found to be shorter than the coupling interval of isolated atrial premature bears. Furthermore, the duration of the PP inrerval preceding the atrial premature beat initiating arrial fibrillation and the isolated atrial premature beats was similar. Although the origin of the initiating atrial premature beat could not be defined in this study, the atrial premature beats did not seem to originate from the defibrillation catheters as suggested by the timing between the earliest atrial endocardial activation on the defibrillation catheters and the onset of the atrial premarure beats on the 12-lead 
ECG. The occurrence of these arrial premature beats together with a very short atrial refractory period immediately after defibrillation ${ }^{8}$ may be responsible for IRAF. Although there was no significant difference in the type of antiarthythmic drug being taken by parients without and with IRAF, one can not exclude that anciarrhythmic drugs may have been responsible in part for atrial premature bears that occurred with significantly shorter coupling intervals when atrial fibrillation was reinitiated.

In patients with IRAF, sinus rhythm was restored with a variety of interventions. First, in all five parients, another defibrillation shock (although with different intensity) was given in an attempt to obtain permanent sinus rhythm. In only two of them long-lasting successful defibrillation was obtained. One can hypothesize that the reason of successful conversion after repeated defibrillation was due to either the deliverance of multiple shocks or higher intensity shocks. It has been shown in ventricular defibrillation that refractory period extension after the shock may facilitate defibrillation in the ventricle? This phenomenon also may have played a role in enhancing defibrillation in the atrium. In the other three patients in whom IRAF could not be prevented by a repeated defibrillation shock, pharmacologic agents were used before repeating internal cardioversion. IRAF was treated successfully with atropine only in one patient. Depression of vagal action on atrial tissue by atropine results in a decrease in conduction velocity and an increase of the refractory period, which may account for its beneficial role in atrial fibrillation. ${ }^{10}$ In the remaining two parients, permanent sinus rhythm was obtained by intravenous flecainide before shock delivery. In a study by Bianconi et al., ${ }^{5}$ pretreatment with Class IC antiarrhythmic drugs significantly decreased the postcardioversion incidence of atrial premature beats as well as the early recurrence of atrial fibrillation.

Recurrences of atrial fibrillation after discharge occurred with a similar incidence and a time interval that was not significantly different in patients with and wirhour IRAF. This stresses the necessity to treat immediate reinitiation promptly to achieve a successful cardioversion.

\section{LIMITATIONS OF THE STUDY}

One of the limitations of the study to validate the different treatment strategies was the small patient population with IRAF. Furthermore, we did not randomize the first shock energy for the treatment of IRAF to determine whether shock strength or the effect of multiple shocks was responsible for successful defibrillation. Nevertheless, methods other than additional shocks to maintain sinus rhythm immediately after successful internal atrial defibrillation need to be explored. This knowledge may enhance its acceptability by eliminating the discomfort of additional cardioversion shocks in patients with an implantable atrial defibrillaror. In the furure, more accurate systems such as those using high-density mapping or a multielectrode cath- 
eter (basket) may be useful to elucidate a unifocal or mulcifocal origin of the atrial premature beats iniriang atrial fibrillation.

In conclusion, IRAF after internal atrial defibrillation occurred in $13 \%$ of patients, was always initiated by an atrial premature bear having a short coupling interwal not originating from the defibrillation catheters, and was prevented by repeared shock delivery with or without preceding administration of pharmacologic agents. IRAF did not predict early recurrences of the arrhythmia after discharge from the hospital, emphasizing the necessity to treat immediate reinitiation promptly to achieve a successful cardioversion.

\section{REFERENCES}

1. Lewy $S$, Lauribe $P$, Dolla $A$, et al: $A$ randomized comparison of external and internal cardioversion of chronic atrial fibrillation. Circulation 1992;86:1415-1420.

2. Baker BM, Botreton GW, Smith JM: Low-energy internal cardiowersion for atrial fibrillation resistant to external cardioversion. I Cardiowasc Electrophysiol 1995;6:44-47.

3. Schmitt C, Alt E, Plewan A, et al: Low energy intracardiac cardioversion after failed conventional extemal cardioversion of atrial fibrillation. J Am Coll Cardiol 1996;28:994-999.

4. Saksena S, Prakash A: Arrial defibrillation: Techniques and dinical results. In: Saksena S, Luderitz B, eds. Interventional electrophysiology. A texthook. Second Edition. Futura Publishing Company, Armonk, NY, 1996,pp. 509 ,520.

5. Bianconi $L$, Mennuni $M$, Lukic $V$, et al: Effects of oral propafenone administracion before electrical cardioversion of chronic atrial fibrillation: A placebo-controlled study. I Am Coll Cardiol 1996;28:700-706.

6. Ayers GM, Alferness CA, Ilina $M$, et al: Ventricular proarrhythmic effects of ventricular cycle length and shock strength in a sheep model of cranswenous atrial defibrillation. Circulation, $1994: 89: 413-422$

7. Capucci A, Santarelli A, Boriani $G$, er al: Atrial premature beats coupling interval determines lone patroxysmal atrial fibrillation onset. Int / Catdiol 1992:36:87-93.

8. Kumagai $K$, Akimitsu $\$$, Kawahira $K$, et al: Elecrophysiological properties in chronic lone atrial fibrillation. Circulation 1991:84:1662-1668.

9. Sweency RJ, Gill RM, Reid PR: Characterization of refractory period extension by transcardiac shock. Circularion 1991:83:2057-2066.

10. Rensma PL. Allessic MA, Lammers WJEP, al: Length of excitation wave and susceptibility to reentrant atrial arthythmias in normal conscious dogs. Circ Res 1988;62:395-410. 
CHAPTER 6

\section{Atrioverter: an implantable device for the treatment of atrial fibrillation}

Hein JJ Wellens, MD; Chu-Pak Lau, MD; Berndt Lüderitz, MD; Masond Akhtar, MD; Albert L Waldo, MD; A. John Camm, MD; Carl Timmemans, MD; Hung lar Tse, MD; Werner Jung, MD; Luc Jordaens, MD; Gregory Ayers, MD; for the Merrix Investigators

From the Academic Hospital Maastricht, Maastricht, the Netherlands (H.J.J.W. C.T.); Queen Mary Hospital, Hong Kong, China (C.-P.L., H.F.T.): University of Bonn, Bonn. Germany (B.L., W.J.); St. Luke's Medical Center, Milwwankee, USA (M.A.); University Hospitals of Cleveland, Cleveland, USA (A.I..W.); Sr. George's Hospital, London, United Kingdom (A.J.C.); Acadenic Hospiral Gent, Gent, Belgium (L.J.); and InControl Ine, Redmond, USA (G.A.).

Abstract presanted in part at the $19^{\text {th }}$ Annual Sctennific Sessions of the Congress of the Europeatn Socievy of Cardiology (Eur Heart J 1997:18,134)

Publisted in Circulation 1998:98:1651-1656 


\section{A B T R ACT}

Background. During atrial fibrillation, electrophysiologic changes occur in atrial tissue that favor the maintenance of the arrhythmia and facilitate recurrence after conversion to sinus rhythm.

An implantable defibrillator connected to right atrial and coronary sinus defibrillation leads allows prompt restoration of sinus rhythm by a low-energy shock. The safery and efficacy of this system, called the Atrioverter, were evaluated in a prospective, multicenter study.

Methods and Results. The study included 51 patients with recurrent atrial fibrillation who had not responded to antiarthythmic drugs, were in New York Heart Association Heart Failure class I or II, and were at low risk for ventricular arthythmias. The atrial defibrillation threshold had to be $\leq 240 \mathrm{~V}$ during preimplant testing. Atrial fibrillation detection, R-wave shock synchronization, and defibrillation threshold were tested at implantation and during follow-up. Shock termination of spontaneous episodes of atrial fibrillation was performed under physician observation. Results are given after a minimum of 3 months follow-up.

During a follow-up of 72 to 613 days (mean, $259 \pm 138$ days), $96 \%$ of 227 spontaneous episodes of atrial fibrillation in 41 patients were successfully converted to sinus thythm by the Atrioverter. In $27 \%$ of episodes, several shocks were required because of early recurrence of atrial fibrillation. Shocks did not induce ventricular arrhythmias. Most patients received antiarthythmic medication during follow-up. In 4 patients, the Arrioverter was remowed: in 2 because of infection, in 1 because of cardiac tamponade, and in 1 because of frequent episodes of atrial fibrillation requiring His bundle ablation.

Concluston. With the Atrioverter, prompt and safe restoration of sinus rhythm is possible in patients with recurrent atrial fibrillation. 
Atrial fibrillation (AF) is the most common sustained cardiac arthythmia! with hemodynamic, thromboembolic, and health and economic consequences. Usually, restoration of sinus rhythm is attempted by pharmacological or electrical therapy ${ }^{2,3}$. In chronic AF, therapy is directed towards maintenance of an acceptable ventricular rate and prevention of thromboembolic complications with anticoagulant therapy. When ventricular rate control cannot be obtained by medication, interruption or modification of $A V$ conduction with or withour ventricular pacing can be performed (4-6). The concept that multiple atrial reentrant wavelets are present ${ }^{7}, 8$ in $\mathrm{AF}$, resulted in the approach to divide the atria into several segments, either surgically (the MAZE operation) or by catheter ${ }^{910}$. In most patients with AF, other cardiovas-

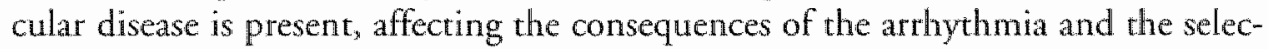
tion of therapy.

In the goat model ${ }^{11}$, persistent AF results in electrophysiological changes in the atrium that favor maintenance of fibrillation and early recurrence after successful conversion. Therefore, to shorten the episode of AF as much as possible seems important to prevent recurrence of $A F$ after conversion to sinus rhythm. This article describes the initial results with an implantable device (the METRIX Arrioverter) that allows rapid and successful conversion of AF by low-energy intracardiac shocks. The objective of our study was to evaluate the safery and efficacy of this new therapy.

\section{METHODS}

\section{PATIENTS}

We enrolled 51 patients from 19 centers in 9 different countries in the study. They had prior episodes of AF that had spontaneously terminated or been converted to normal sinus rhythm with intervals of recurrence of AF episodes between 1 week and 3 months. Treatment with at least 1 class I or III antiarrhythmic drug was ineffective or only partially effective, resulting in arrhythmia recurrences, or nor rolerated because of side effects. Exclusion criteria are given in Table 1. The 51 patients underwent clinical evaluation, including history and physical examination, 12-lead ECG, chest radiography, echocardiography, and laboratory tests. The protocol received approval from the ethics committee or institutional review board of each participating center. Informed consent was obtained from each patient.

\section{THE DEVICE}

The InControl METRIX Atrioverter system consists of an implantable atrial defibrillator (model 3000 or 3020) connected to right atrial (perimeter right atrial model 7205) and coronary sinus (perimeter coronary sinus model 7109) defibrillation leads and a bipolar endocardial ventricular pacing lead (Figure 1), a 
Table 1. - Exclusion criteria for arral defibrillator implant.

- Implanted heart valve

- Al of reversible cause (og afrer cardiac surgery, hyperthyroidism)

- Wolff-Parkinson-White synd rome

- Myocardial infarction or myocardial revascularizarion $\leq 1$ year.

Active angina or cardiac ischemia

- History of left arral thromboembolic events.

- Congestive heart failure

- Left ventricular ejection fraction of $<40 \%$

- ECG documentarion of sustained or nonsustained ventricular tachycardia ( 3 consecutive beats)

Defibrillation level of $>240$ Volts at unclusion testing procedure.

programmer, and a defibrillation systems analyzer. The defibrillator is a battery-powered, pectorally implanted device with a displacement of $53 \mathrm{~cm}^{3}$ and a weight of $79 \mathrm{~g}$ (model 3000) or $82 \mathrm{~g}$ (model 3020). With specific algorithms, the device derects AF and delivers R-wave synchronous defibrillation shocks to convert AF to sinus rhythm. It is also able to pace the ventricle after shock delivery in case of bradycardia. Shocks can be delivered at a selected voltage, with a maximal intensity of $300 \mathrm{~V}$. The model 3000 defibrillator has an $80-\mu \mathrm{F}$ capacitor and can delliver a maximal shock of $3 \mathrm{~J}$ with a biphasic waveform of $3 \mathrm{~ms} / 3 \mathrm{~ms}$. The model 3020 defibrillator has a $160-\mu \mathrm{F}$ capacitor with a maximal shock of $6 \mathrm{~J}$ with a biphasic waveform of $6 \mathrm{~ms} / 6 \mathrm{~ms}$. The device stores the intracardiac electrograms from the last 6 successfully terminated episodes of AF. Real-time telemetry of 3 simultaneous intracardiac electrograms can be obtained via the programmer. The device can be programmed in an automatic mode with automatic, periodic acrivation of the detection algorithm and shock delivery after a preset delay from onser of AF, or it can be used in a patient-or physician-activated mode in which the detection algorichm and shock therapy are initiated by placing a magnet over the Atrioverter. During this study, the device was programmed in the monitor mode, and shock therapy delivered in hospital by a physician.

\section{STUDY PROTOCOL}

\section{Preimplant, inplant, and postimplant procedures}

During the patient inclusion testing procedure, either temporary catheters or permanent leads were placed in the coronary sinus, right atrium, and the right ventricle. Patients were sedated according to the normal dinical practice of each institution. Right ventricular pacing and sensing thresholds were obtained. All leads were connected to the defibrillation systems analyzer or model 3020 device fixture. Atrial signals were examined by performing the AF detection algorithm. After AF detection 


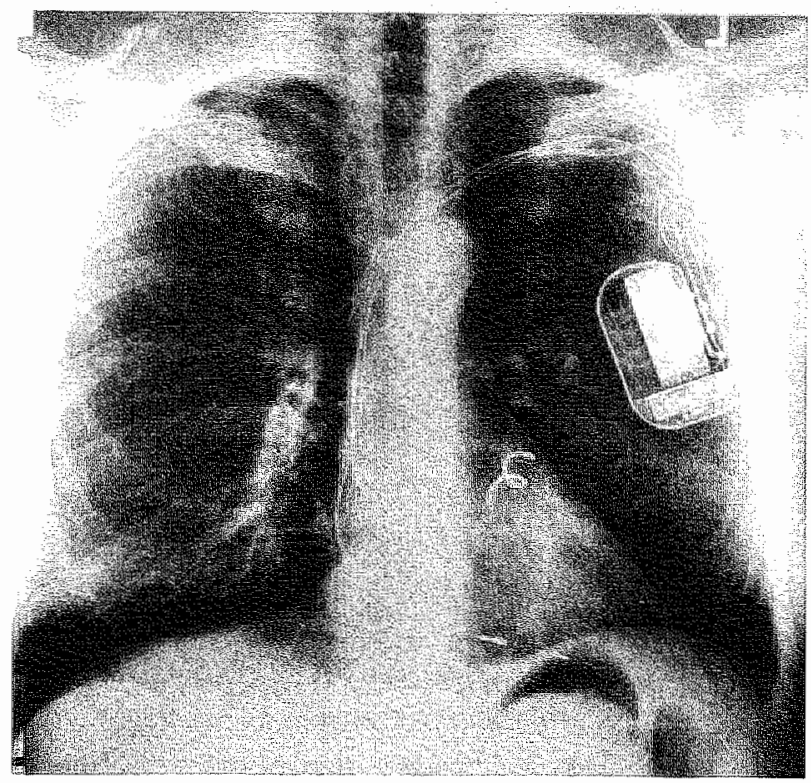

Figure 1. - Chest roentgenogram showing Atrioverter with leads in right atrium, coronary sinus, and apex of right ventricle. Right atrium and coronary sinus leads are used for arthythmia recognition and defibrillation. Right ventricle lead is used for shock synchronization and, if needed, ventricular pacing.

testing, the synchronization algorithm was rested. Synchronization markers were examined during sinus rhythm to ensure that the device was synchronizing properly with the ventricular depolarization. When proper synchronization was confirmed, the device was programmed to deliver shocks synchronized to the $\mathrm{R}$ wave during sinus rhythm to induce AF. If the device did not induce AF, trapid atrial pacing was used as an alternative induction method. The AF detection and synchronization tests were also performed during AF. To be eligible for implantation, 2 successes at $240 \mathrm{~V}$ had to be obtained during resting. This was later revised to allow 1 success out of 3 attempts at $260 \mathrm{~V}$ for model 3000 and $240 \mathrm{~V}$ for model 3020 .

At implantation, permanent leads were placed in the coronary sinus, right atrium and the right ventricle. Right ventricular pacing and sensing thresholds were obtained. AF derection and R-wave synchronization were again assessed. Two of 4 successes at $240 \mathrm{~V}$ had to be attained at the final lead location. Atrial defibrillation threshold (ADFT) was estimated as follows. Starting with a 180-V shock, the shock intensity was increased in $20-\mathrm{V}$ steps until successful defibrillation was achieved. After this initial success, AF was reinduced and a shock $20 \mathrm{~V}$ less than the previously successful shock was given. Thereafter, the shock intensity was decreased in $20 \mathrm{-V}$ steps until a shock intensity was delivered that failed to convert the AF despite the delivery of 2 shocks ar this intensity. Shocks were then delivered at $20-\mathrm{V}$ steps of 
increasing in tensity until AF was successfully converted with the delivery of 2 shocks at this intensity.

To implant the device, ADFT had to be $\leq 240 \mathrm{~V}$. Automatic mode operation, which consisted of AF detection, capacitor charge, AF redetection, synchronization, shock delivery, postshock pacing (if needed), and AF detection, was also tested during the implant procedure.

Postimplant evaluation was performed at pre-discharge; 1,3 , and 6 months; and at 6-months intervals thereafter untill the completion of the study. AF detection and $\mathrm{R}$-wave synchronization tests were performed at predischarge and at 1 - and 3 -month follow-ups. The defibrillation level was tested during the predischarge and at 1-month follow-up. Long-term ADFT testing was repeated at the 3-month follow-up. Follow-up witl device interrogation was performed at 6 months and every 6 months thereafter. At those times, AF was induced during sinus rhythm by low-intensity shocks or by rapid atrial pacing by use of a separate catheter. AF was permitted to last for $\geq 5$ minutes before test shocks were given.

During preimplant, implant, and postimplant shock delivery, cardiac thythm was continuously recorded to document the possible induction of ventricular arrhythmias. The decision to use anticoagulant therapy in the preimplant, implant and postimplant period was left to the investigator, with protocol suggesting the use of anticoagulant therapy as if the patient did nor have the device.

\section{Treatment for spontaneous episodes}

When the parient sought treatment of a spontaneous episode, data pertaining to shock effectiveness were recorded. Clinical factors, such as antiarrhythmic medications and changes in system performance in case of lead position changes, were also recorded.

\section{RESULTS}

After the first implant on October 31, 1995, 51 parients received the Atrioverter, and all parients had $\geq 3$ months of postimplant follow-up as of Jully 4, 1997. These 51 patients were selected from 119 patients undergoing a screening testing procedure. Of 50 patients scteened, 17 received the model 3000 , and 34 of 69 screened patients received the model 3020 . Of the failed screenings, 43 were due to high ADFT. The remaining patients did not proceed to implant because of either physician/patient decision or exclusions for enrollment. Patient demographics are given in Table 2. Two patients had a history of PTCA, and 2 patients had had CABG. All patients had been treated with $\geq \mathbb{1}$ class I or III antiarrhythmic drug (average, 3.9 drugs) that had been discontinued, was not tolerated, or was only partially effective. The average duration of follow-up was $259 \pm 138$ days (approximately 8.6 months), with a median of 232 days (approximately 7.7 months). 
Table 2.- Profile of the 51 parients studied.

\begin{tabular}{ll}
\hline Sex & 40 men, 11 women \\
Age & $31-77$, mean 58 years \\
Average number of AADs & 3.9 \\
LVEF" & $38-87$, mean $58 \pm 11 \%$ \\
LA size & $3.3-6.6$, mean $4.4 \pm 0.8 \mathrm{~cm}$ \\
NYHA class & class $1: 36$ patients \\
& class II: 15 parients
\end{tabular}

AAD indicates anti-arrhythmic drug; LA: left atrium; LVEF: left ventriculat ejecrion fracrion: * Measured during sinus rhythm.

\section{SAFETY}

A total of 3719 shocks were delivered: 3049 during testing and 670 for spontaneous episodes of AF. All shocks for spontaneous episodes were given during physician observation. There were no reported cases of induction of ventricular arrhythmias or inaccurately synchronized shocks during the study. Analysis of the AF derection algorithm performance during observed operations revealed a $100 \%$ specificity for the recognition of sinus rhythm (as opposed to AF) and $92.3 \%$ sensivivity for the detection of $\mathrm{AF}$ (as opposed to sinus rhythm). A rotal of 1062 tests of the detecrion algorithm were performed in sinus rhythm and $1178 \mathrm{in} \mathrm{AF}$.

\section{EFFICACY}

\section{Treatment of spontaneous episodes}

Forty-one patients had 231 episodes of AF for which they sought therapy (average, 5.6 episodes per patient; range, 1 to 26 episodes). Four episodes of AF occurred immediately before the time of threshold testing, and these episodes were used for clinical testing of the device. Because these episodes were not treated as spontaneous episodes bur rather were used for this testing, they are excluded from further analysis and discussion. We delivered 670 shocks for the treatment of the remaining 227 episodes (median, 3 shocks per episode). The device terminated $96 \%$ of the episodes, I episode spontaneously converted before complete device therapy delivery, and 10 episodes (in 9 patients receiving a total of 48 shocks) could not be converted. Seven of these episodes later spontaneously converted, 2 were chemically cardioverted, and 1 was converted with external cardiowersion after antiarthythmic drug pre-treatment.

Early recurrence of AF (ERAF) (Figure 2) was present during the treatment of 62 episodes (27\% of all episodes) and seen in 21 of 41 patients (51\%). ERAF was defined as the resumption of AF within 1 minute after a shock that resulted in sinus: rhythm for $\geq 1$ beat. This prevented restoration of persistent sinus thythm for 26 of 


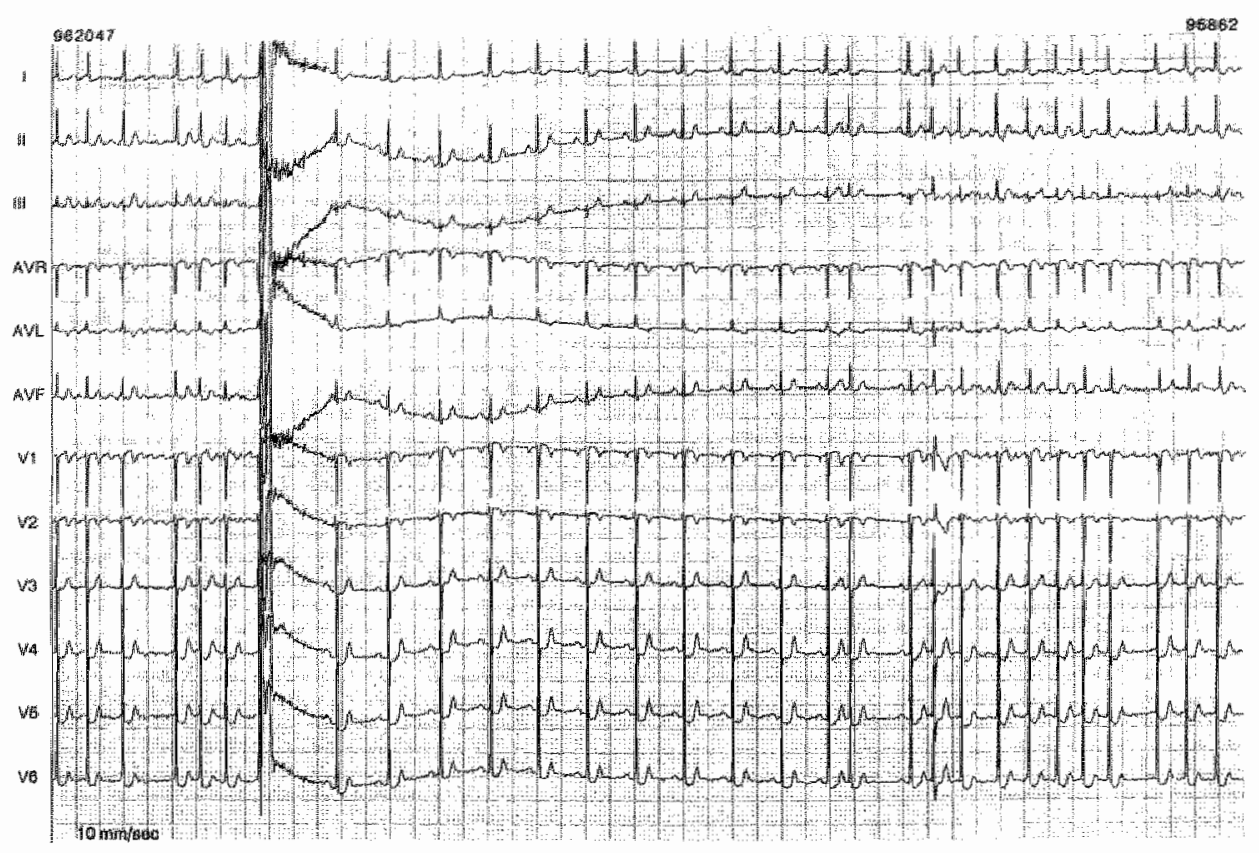

Figure 2, - Example of early recurrence of AF after a successful shock-induced conversion to sinus rhythm.

the episodes. Six episodes were chemically converted before additional shock delivery, 1 was converted with external defibrillation, and 19 were allowed to convert spontaneously at a later time. For 22 episodes in which stable sinus rhythm was eventually obtained, antiarthythmic drugs were injected intravenously followed by repeated cardioversion. With ERAF taking into account, the overall clinical efficacy of the device was $86.3 \%$.

One patient with frequent episodes ( 1 to 2 per week) and a drug-resistant rapid ventricular rate underwent $H$ is bundle ablation and permanent ventricular pacing. One patient had a failed conversion attempt before the 1-month follow-up and underwent successful lead repositioning with the next spontaneous episode and subsequent spontaneous episodes successfully terminated with the device. Three patients had successful conversions early but experienced late failure. In 1 of these patients, spontaneous episodes were successfully converted after repositioning of the right atrial electrode. One patient had a high implant threshold, and $50 \%$ of the episodes could be converted after implantation.

Ten patients did not receive Atrioverter treatment for spontaneous episodes of AF. As shown by examination of the episodes $\log , 5$ patients had no episodes of AF (follow-up duration, 58 to 354 days; mean, $164 \pm 112$ days). Five patients did have episodes of AF (1 parient had 1 episode, 1 had 2,1 had 5, 1 had 6, and 1 had 15 ) during a follow-up of 52 to 382 days (mean, $177 \pm 124$ days). Because they did not go 


\section{VOLTS}
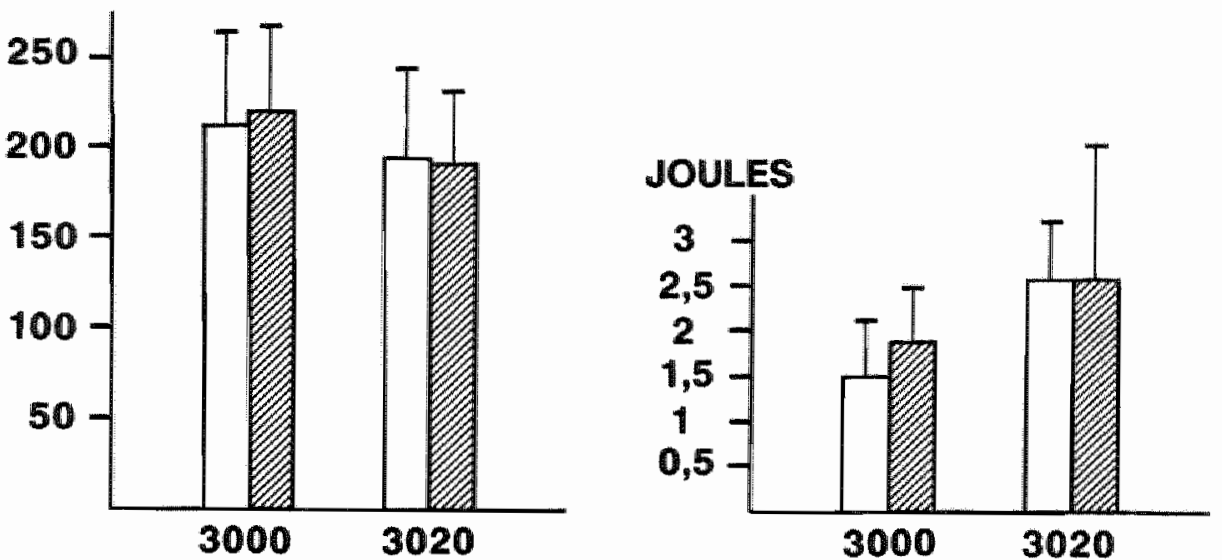

Figure 3. - ADFTs at Atriowerter implant and 3 months later. Shown are data expressed as mean witl corresponding SD from models 3000 and 3020 .

to a hospital, no Atrioverter treatment of these episodes was given. All 47 patients having their device at the end of follow-up were in sinus rhythm at that time.

\section{Atrial defibrillation thresholds}

ADFTs were measured at implantation and at the 3-month follow-up. The data collected were the shock voltage and energy of 2 consecutive successes and 2 consecutive failures; the average of these 2 values was defined as the ADFT. As shown in Figure 3, ADFTs were slightly higher at 3 months for the 3000 model and slightly lower in the 3020 model, without reaching statistical significance.

\section{Antiarhythmic medication after implantation}

All but 3 the patients who entered the study were on antiarthythmic medication at the time of trial closure. At implantation, 27 patients were on D-L sotalol (18 patients) or amiodarone ( 9 patients). These numbers were 18 and 15 patients, respectively, at the end of the study.

\section{Shock tolerability}

Use of sedation preceding shock delivery was left to the treating physician in conference with the patient. Marked variability in shock tolerance between parients was reported by the investigators. The interinstitutional variability in the level of sedation used made it impossible to address the shock tolerance issue in detail. 


\section{COMPLLCATIONS}

Two parients with the device implanted had subclavian venous thrombosis. One was treated successfully with urokinase. In the other patient, it was considered to be a chronic problem, and no intervention was performed. In 1 patient, a pericardial effusion deweloped a few hours after testing during the 1 -month follow-up study. During the testing session, 19 shocks were delivered, 17 ar low voltage for induction of $A F$ and 2 for defibrillation. When the patient returned to hospital because of chest pain, shortness of breath, and hypotension, the echocardiogram revealed cardiac tamponade. At operation, perforation of the atrial wall by the right atrial electrode was found. The right atrial and right ventricular leads were extracted, and the right atrial perforation site repaired. The coronary sinus lead and the Atrioverter were removed a few days later. The patient made an uneventful recovery. Post hoc review of the serial chest $x$-rays from the 4-day postimplant period revealed that the lead was floating in the atrium on postimplant day 1 . The unstable position of the right atrial lead probably resulted in atrial perforation during repeated shock applications 1 month after implantation. Two parients developed infection, requiring explantation of the device and leads.

Four parients required repositioning of the right atrial lead: 1 patient because of lead dislocation and 3 because of an acute increase in the ADFT. One patient had dislodgement of the right ventricular lead that resulted in a change in signal quality, inhibiting appropriate shock delivery. The lead was successfully repositioned.

\section{DISCUSSION}

Thirty-five years ago, Lown et al ${ }^{2}$ showed that $\mathrm{AF}$ could be converted to sinus rhythm by a transthoracic high-energy shock. In 1970, Jain et al ${ }^{12}$ demonstrated that $\mathrm{AF}$ could be treated successfully by giving shocks with 1 electrode in the right atrium and the orher over the chest wall. In 1992, Lévy et al ${ }^{3}$ reported that a high-energy shock given within the atrium had a higher immediate success rate than transthoracic defibrillation. More recently, it was shown that the energy required to defibrillate could be markedly reduced by the use of large-surface right atrial and coronary sinus electrodes and biphasic shock waveforms. ${ }^{13-23}$ The present study was designed to determine the safery, efficacy, and complications of an implanted device able to recognize AF and to treat the arrhythmia by delivery of an appropriarely timed defibrillation voltage. To prevent a possible long period of ventricular electrical standstill after successful defibrillation, the device was equipped with an on-demand ventricular pace function. A large number of defibrillation shocks were given, both during the testing phase (3049 shocks) and during attempts (670 shocks) to convert spontaneous episodes of AF during a follow-up period of 72 to 613 days (mean, $259 \pm 138$ days). In all parients, the shocks were given under the 
observation of a physician. No wentricular arthythmias were induced in any parient, indicating that the device was safe in the patient population studied. This safery record, ie, the absence of inadvertent precipication of a ventricular tachyarrhythmia, reflects the application of the lessons learned from prior testing in animal models $13-18$ and parients. ${ }^{18-21}$ This resulted in programming the atrial shock synchronized to a QRS complex, which occurs $\geq 500 \mathrm{~ms}$ after the preceding QRS complex, to avoid the $T$ wave of the preceding bear. Such an approach is possible because conversion of $A F$ is not an emergency.

Efficacy of the device was evaluated by determining its ability to recognize $\mathrm{AF}$, to reject sinus thythm, and to convert AF to sinus thythm by a low-energy shock. Arthythmia recognition occurred with high specificity. In concrast to the ventricular defibrillator in which the life-threatening character of the arrbythmia requires a short time from onset to therapy and therefore a high sensitivity, the atriall defibrillator, which is treating a non-life threatening arthythmia, should be able to recognize $A F$ with a very high degree of specificity. The device was effective in terminating $A F$, restoring $74 \%$ of episodes to sinus rhythm without further intervention. With drug intervention for ERAF or failed conversion, the overall efficacy of the device was $86 \%$. As discussed elsewhere, ${ }^{24}$ ERAF within minutes after a successful cardioversion is an important problem. They were observed in our patients in $27 \%$ of episodes and occurred at least once in approximately half the patients. About half the time, it could be controlled by the intravenous administration of anriarrhythmic drugs, followed by repeated cardiowersion. This might present a problem when the device is used outside the hospital. Not only control of ERAF but also the tolerance of the defibrillator shock are important aspects of a long-term success of the implantable Atrioverter. As shown by Ammer et $\mathrm{al}_{*}{ }^{25}$ whereas shock energy determines the success of a shock, shock voltage determines the pain perceived by the patient. By using waveforms that deliver greater energy at lower peak voltages, interna cardioversion of AF can be performed with less sedation and greater parient tolerance. The discomfort from intracardiac low-energy shocks is still poorly understood. Most investigarors $18,25-27$ found marked individual variations in shock tolerabiliry. The number of shocks is important, with discomfort growing as the number of shocks increases. This suggest that the first shock should be well above the ADFT to make the shock successful. Lok et al ${ }^{26}$ showed that most patients can rolerate a shock level of $260 \mathrm{~V}$ without sedarion.

It is well known that longer-lasting AF is more difficult to convert to sinus thythm and has a higher incidence of recurrences. $23,28,29$ It has been shown in the animal model that electrophysiological remodeling of the atria leads to marked shortening of the atrial refractory period and a loss of the normal relation between heart rate and the duration of the atrial refractory period favoring ERAF. II

If this electrophysiological remodeling of the atria can be prevented by defibrillation as early as possible, it is likely that recurrences of AF will decrease and the number of necessary shocks will diminish over time. Careful follow-up of the 
number of episodes of AF afrer implantation of the Arrioverter should answer that question.

The complications observed were mostly related to the use of intracardiac catheters and the implantation of a device, such as the necessity to reposition the catherer (6 patients) and infection ( 2 patients). In 1 patient, His bundle ablation and pacemaker implantation was performed because of very frequent episodes of AF with a high ventricular rate. A serious complication, arrial perforation with cardiac amponade, occurred in 1 patient. In retrospect, inappropriate fixation of the atrial lead seems to be the explanation of this complication. The relarively high complication rate is probably related to the learning curve of using the device but may also be caused by its use in 19 centers with differences in techniques, management, and experiences. This resembles the carly implantable ventricular defibrillator experience. 30

\section{CONCLUSIONS}

The implantable Arrioverter recognizes AF with high specificity, and in the patients studied, low-energy defibrillation shocks can be given safely and effectively, resulting in prompt restoration of sinus rhythm. Early recurrences of $A F$ after a successful shock occurred at least once in 21 of the 41 parients and usually required the addivional administration of antiarrhythmic drug therapy. Shock tolerance waried markedly between parients. Lowering of the ADFT by changing catheter characreristics and shock wave form and the availability of a short-acting, nonaddictive analgeticlanxiolytic drug should facilitate the acceptance of this device. The patients studied fulfilled strict selection criteria, and the performance of the device was observed in hospital. Several pharmacological and nonpharmacological treatment options are currently available to patients with AF. The proper place of the implantable Atrioverter in the treatment of AF requires further investigation.

\section{REFERENCES}

1. Kannel WH, Abbot RD, Savage DD, McNamara PM. Epidemiologic features of atrial fibrillarion: the Framingham Study. N Engl I Med. 1982;306:1018-1022.

2. Lown B. Amarasinghan $R$. Neuman I. New method for teminating andiac arthythmias. Use of synchronized capacitor discharge. JMA. 1962;182:548-555.

3. Léwy S, Lauribe P, Dollia E. Brémondy M, Calkins H, Das S, Morady F. A randomized comparison of exremal and internal cardioversion of chronic atrial fibrillation. Circulation. 1992;86:14151420.

4. Scheinman M, Morady F, Hess. D, Gonzalez R. Catheter induced abiation of the atrioventricular juncrion to control refractory supraventricular arrhythmias. JAMA. 1982:24:8:851-855. 
5. Gallagher J, Svenson R, Kassel J, German LD, Bardy GH, Broughton A, Critelli G. Catheter rechnique for closed-chest ablacion of the arroventricular conduction system: a therapeucic alternative for the treatment of refractory supraventricular rachycardia. N Engl I Med. $1982 ; 306: 194-200$.

6. Willamson BD, Man KC, Daoud E, Niebauer M. Strickberger SA, Morady F. Ratiofrequency catheter modification of atriowentricular conduction to control the ventricular rate during atrial fibrillation. N Engl J Med. 1994;331:910-917.

7. Moe GK. On the multiple wavelet hypothesis of atrial fibrillarion. Arch Int Pharmacodyn Ther. $1962 ; 140: 183-188$.

8. Allessie MA, Rensma IPL, Brugada J, Smeets JRLM, Penn O, Kirchhof CJHJ. Parhophysiology of atrial fibrillation. In: Zipes DP, Jalife J, eds. Cardiac electrophysiology: From Cell to Bedside. Philadelphia, Pa: WB Saunders Co; 1990:548-558.

9. Cox I, Boineau J, Schuessler R. A rewiew of surgery of arrial fibrillation. I Cardiovasc Electrophysiol. 1991;2:541-561.

10. Haissaguerre M. Right and left anial radiofrequency carherer therapy of paroxysmal atrial fibrillation.] Cardiovase Electrophysiol. 1996\%7:1132-1144.

11. Wijffels MCEF, Kirchhof CJHJ, Dortand R, Allessie MA. Arrial fibrillation begers atrial fibrillation: a study in awake chronically instrumented goats. Circulation. 1995;92:1954-1968.

12. Jain 5C, Bhatnagar WM, Azaml RU, Awasthey P. Elective countershock in atrial fibrillation with an intracardiac electrode: a preliminary report. J Assoc Physicians India. 1970;18:821-826.

13. Powell AC, Garan H, McGovern BA, Fallon IT, Krishnan SC, Ruiskin JN. Low energy conversion of atrial fibrillation in the sheep. J Am Coll Cardiol. 1992;20:707-711.

14. Cooper RAS, Alferness CA, Smith WM, Ideker R. Internal cardioversion of atrial fibrillation in sheep. Circulation. 1993;87:1673-1686.

15. Ayers GM, Alferness CA, Ilina M, Smith WM, Cooper RAS, Ideker R. Ventricular proarrhydhmic effects of ventricular cycle length and shock strengrh in a sheep model of transvenous atrial defibrillarion. Circulation. 1994; 89:413-422.

16. Sokoloski MC, Ayers GM, Kumagai K, Khrestian CM, Niwano S, Waldo AL. Safety of transvenous arrial defbrillation: studies in the canine sterile pericarditis model. Circulation. $1997: 96: 1343-1350$.

17. Keelan ET, Krum D, Hare J, Mughal K, Li H, Akhtar M, Jazayeri MR. Saffety of alrial defibrillarion shocks synchronized to narrow and wide QRS complexes during atrial pacing prorocols simularing atrial fibrillarion in dogs. Circulation. 1997:96:2022-2030.

18. Alt E. Schmit C, Ammer R, Coenen M, Fowhi P. Karch M, Blasini R. Initual experience with intracardiac atrial defibrillation in patients with chronic atriall fibrillation. Pacing Clin Elecrophysiol. 1994;17:1067-1.078.

19. Saksena S, Prakash A, Mangeon L, Varanasi S, Koletris Th, Mathew Ph. De Groot P, Melura R, Krol RB. Clinical efficacy and safery of arrial defabrillation using biphasic shocks and current nonthoracotomy endocardial lead configurations. Am J Cardiol. 1995;76,913-921.

20. Murgarroyd F, Slade AKB, Sopher M, Rowland E, Ward DE, Camm AJ. Efficacy and tolerability of transwenous low energy cardiowersion of paroxysmal atrial fibrillation in humans. I Am Coll Cardiol. 1995:25:1347-1353. 
21. Sra JS, Maglio C. Dhala A, Jazayeri MR, Akhtar M. Feasibility of acrial fibrillarion detection and use of a preceding synchromization interval as a criterium for shock delivery in humans with atrial fibrillation. J Am Call Cardiol. 1996; 28: 1532-1538.

22. Schmit C. Al E. Plewan A, Ammer R, Leibig M, Karch M, Schömig A. Low energy intracardiac candiowersion after failed conventional exremal cardioversion of atrial fibrillation. I Am Coll Cardial. 1996:28:994-999.

23. Lévy S, Ricard P, Lau CP, Lok NS, Camm AJ, Murgutroyd FO, Jordaens LJ, Kappenberger Ll, Brugada P, Ripley KL. Multicenter low energy transvenous atrial defibrillation (XAD) trial results in different subsets of atrial fibrillation. IAm Coll Cardiol. 1997;29:750-755.

2.4. Timmermans $C$, Rodriguex LM, Smeets JLRM, Wellens HJJ. Immediate reiniriation of atrial fibrillation following internal atral defibrillation. f Cardiovasc Electrophysiol. 1998;9: 122-128.

25. Ammer R, Alt E, Ayers G, Schmüt C. Pasquantonio J, Schmirdr M, Püter $K_{x}$ Schömig A. Pain threshold for low energy intracardiac cardioversion of atrial fibrillation with low or no sedation. Pacing Clin Electrophysioll. 1997:20:230-236.

26. Lok NS, Lau CP. Tse HF, Ayers GM. Clinical shock tolerability and effect of different right atrial electrode locations on efficacy of low energy human transvenous atrial defibrillation using an implantable lead system. I Am Coll Cardiol. 1997; 30: 1324-1330.

27. Jung J, Heisel A, Fries $\mathbb{R}$, Kölner $V$. Tolerability of internal low-energy shock strengths currently needed for endocardial atrial cardioversion. Am J Cardiol. 1997; 80: 1489-1.490.

28. Van Gelder IC, Crijns $H$ J, Van Gilst WH, Verwer R, Lie KI. Prediction of uneventful cardioversion and maintenance of sinushythm from direcr-current electrical cardioversion of chronic atrial fibrillation and flutter. I Coll Cardiol. 1991; 68:41-46.

29. Van Gelder IC, Crijns HJ, Tieleman RG, wan Gilst WH, Lie KI. Chronic atrial fibrillation: success of serial cardiowersion therapy and safery of oral anticoagulation. Arch Intern Med. 1996; 156: 2585-2593.

30. Marchlinski FE, Flores BT, Buxton AH, Miller J, Josephson ME. The automatic implantable cardioverterdefithillaror: efficacy, complicarions and device failures. Ann Intern Med. 1986; 104: $481-490$

\section{APPENDIX}

\section{LIST OF PARTICIPATING CENTERS AND INVESTIGATORS}

Acudemic Hospital Gent, Gent, Belgrium (L. Jordacns, R. Tavernier, F. Provenier); Hópiral Nord Marseille, Marseillle, France (5. Lévy, V. Taramasco, E. Dolla); Hôpital Cardiologique, Lille, France (S. Kacer, D. Lacrơx, P. ke Franc, D. Klug, C. Kouakam); Hôpiral "Hôtel Dieu", Rennes, France (C. Daubert, D. Gras, P. Mabo, D. Paxin); Unversiry of Bonn, Bonn, Germany (B. Lüderitz, W. Jung); Klinikum der Sadt Ludwigshafen, Ludwigshafen, Germany (K. Seidl); Acadernic Hospiral Maastricht, Maastricht, the Netherlands (H. Wellens, C. Timmermans, L.M. Rodriguez); Academic Hospitat Groningen, Groningen, the Netherlands (H. Crijns, I. van Gelder); Sahlgrenska Sjukhuser, Göteburg, Sweden (N. Edwardsson, L. Lurje, M. Aunes, H. Walfricsson); Karolinska Hospital, Stockholm, 
Sweden (M. Rosenquist, C. Linde); St. George's Hospial, London, Unired Kingdom (A.j) Camm, E. Rowland, D. Ward, S.M. Sopher, M. Gallagher, A. Slade, J. Waktare); The Queen Mary Hospital, University of Hong Kong, China, (C.I. Lau, H.F. Tse, N.S. Lok, K. Lee): University of Calgary, Calgary, Canada (D.G. Wyse); Institute of Cardiology, Montréal, Canada (B. Thibalut, M. Talajic) St. Michael's Hospital, Toronto, Canada (P. Dorian, D. Newman), St. Luke's Medical Center, Milwankee, Wis, USA O. Sra, M, Akhrar, M. Miehl, Z. Blanck, S. Deshpande, A. Dhala, H. Jazayeri. D. Kress); Universiry Hospirals of Cleveland, Cleveland, Ohio, USA (L. Biblo. J. Mackall. A. Waldo. D. Rosenbaum, M. Cartson); University Hospital, Ann Arbor, Mich, USA (A. Strickberger, F. Morady, E. Daoud, R. Goyal, M. Harvey, K. Ching Man); and Uniwersity of Utah Medical Center, Salc Lake Ciry, Utah, USA (R. Freedman, R. Klein, J. Mason, G. Muetheims, P. Specror, S. Compron). 


\section{Spontaneous episodes of atrial fibrillation after implantation of the Metrix ${ }^{\top M}$ Atrioverter: observations on treated and nontreated episodes}

Carl Timmermans, MD; Samuel Lévyt. MD; Gregory M Ayers" MD; Werner Jungs, MD; Luc Jordaensilf, MD; Marten Rosenquist 9 , MD; Bernard Thibault MD; John Camm $^{\text {*** }}$, MD; Luz-Maria Rodriguez*, MD; Hain J) Wellens*, MD; for the Merrix Investigatorst

From the *Academic Hospital Maastricht, Maastricht, the Netherlands; tHopital Nord Marseille, Marseille, France; $¥$ InControl Inc, Redmond, WA, USA; $\$$ University of Bonn, Bonn, Germany; |fUniversity Hospital Gent, Gent, Belgium; SKarolinska Hospital, Stockholm, Sweden; \#Montreal Heart Institute, Quebec, Canada; and *'St. George's Hospital, London, United Kingdom. †tThe Merrix Investigators are listed in the Appendix. 


\section{ABSTRACT}

Objectives. To evaluate the number and duration of device-treated and self terminating, nontreated episodes of atrial fibrillation (AF) after implantation of the Merrix ${ }^{T M}$ Arriowerter.

Backgrownd. A recent study has shown that the Atrioverter can rapidly restore sinus thythm in patients with AF, however the effect of the device on the clinical course of the arrhythmia in these patients is unknown.

Methods. The Atrioverter was implanted in 51 patients with symptomatic, recurrent, drug-refractory AF. The device was programmed to periodically monitor the cardiac rhythm. Defibrillation of AF episodes was performed under physician observation. Results. During a mean follow up of $260 \pm 144$ days, 1161 episodes of AF were observed during walid monitoring periods in 45 of 51 patients. Forty-one patients experienced 231 episodes for which they sought defibrillation therapy. The average duration of the treated episodes during vallid monitoring periods (190/231 episodes from 39/41 patients) was significantly longer than the nontreated episodes (38 \pm 44 vs $10 \pm 8$ hours; $0<0.05$ ). The time berween episodes requiring Atrioverter therapy lengthened and the risk of having an episode requiring trearment decreased. No changes were observed in the number and duration of the short-lasting, nontreated episodes as rime since implant of the device increased.

Conchroms. In patients with symptomatic, recurrent, drug-refractory $\mathrm{AF}$, the frequency of long-lasting episodes, which were treated under obserwation with repeated defibrillarion using the Atrioverter, decreased. The number and duration of short-lasting, nontreated episodes did not change during the 20 month study period. The effect of ambulatory use of the device on the recurrence of short-lasting episodes needs to be evaluated.

Abbroviation. $\mathrm{AF}=$ atrial fibrillation 
Several non-pharmacological options are currently available to treat patients with AF. Internal atrial defibrillation using percutaneous transvenous catheter electrodes has been shown to be effective and safe in converting AF to sinus rhythm, using $R$ wave synchronized low energy shocks ${ }^{1-3}$. These observations resulted in the devalopment of an implantable atrial defibrillator (Metrix TM Atrioverter, InControl Inc., Redmond, WA). The Atrioverter was recently evaluated in a prospective, multicenter study with as primary objective the safery and efficacy of the dewice ${ }^{4}$. The Atrioverter, however, is not only able to safely treat episodes of AF, but also to provide information about the incidence and duration of episodes. The aim of this study was to report on a) the occurrences of AF episodes, treated and nontreared by the Atrioverter and b) the effect of repeated intraatrial defibrillation on the subsequent AF episodes.

\section{METHODS}

\section{PATIENTS}

From October 1995 until July 1997, the Atrioverter was implanted in 51 patients with prior episodes of AF that spontaneously terminared or were converted to sinus rhythm with intervals of recurrence between 1 week and 3 months. Prior ineffective treatment with at least 1 Class I or III antiarrhythmic drug was required. The exclusion criteria of this prospective, multicenter study have been described previously ${ }^{4}$. Concomitant pharmacologic trearment (e.g., anticoagulation, antiarthythmic drugs) was left to the preference of the physician. The Metrix ${ }^{\mathrm{TM}}$ model 3000 was implanted in 17 patients and the Metrix TM model 3020 in the remaining 34 patients. These two devices differ only in defibrillation waveform and output ${ }^{4}$.

Patients were instructed to come to the hospital for treatment of each symptomatic episode of $\mathrm{AF}$ where defibrillation was then performed under physician observattion. Data pertaining to defibrillation therapy, such as shock chatacteristics and effectiveness; and clinical factors, such as anticoagulation and antiarrhythmic medication, were recorded. The protocol was approved by the Ethics Committee or Institutional Review Board of each participating center. Written informed consent was obtained from each patient.

\section{FOLLOW-UP}

The study ended after the last patient implanted completed his three-month, postimplant follow-up. Postimplant clinical follow-up with device interrogation was performed at pre-discharge, one-month, three-months, six-months and at six months intervals thereafter until the completion of the overall study. When the 
patient sought treatment of a spontaneous episode, device interrogation was also performed.

\section{AF EPISODE DEFINITION AND DETERMINATION}

Discrete sampling of the cardiac rhythm was performed by the device on periodic intervals. The device was programmed to perform an AF derection every 20 minutes ro 1 hout. Each time AF was detected, notation of the date and the time of the individual detection was logged to memory. Valid monitoring periods were defined as the time bewwen interrogations of the device where a printout of the interrogation was made and the device memory did not overflow due to too many individual AF detections during this inter-interrogation period (i.e., greater than 170 individual AF detections). From the individual decections of AF, the onset and the duration of all episodes during valid monitoring periods were reconstructed.

Recently, Tse et al. reported the long-term efficacy results of the AF detection algorithm of the Atrioverter and demonstrated that the algorithm had $100 \%$ specificity and $92.3 \%$ sensitivity for the detection of AF in the same patient population ${ }^{5}$. As specificity was $100 \%$, the onset of an episode was defined by a single AF detection. As sensitivity was $92.3 \%$, we required at least 3 consecutive detections as "nor AF" before defining the end of an episode. In theory, this method increased the sensitivity to detect the end of an AF episode to more than 99\%. Lastly, we excluded any patent where the individual AF detection function did not meet a $100 \%$ specificity and $>90 \%$ sensitivity cut-off.

\section{STATISTICAL ANALYSIS}

Continuous variables are expressed as a mean and standard deviation. The overall goal of this study was to determine if the data supported the hypothesis that the duration between treated AF episodes increases as a function of time from implant of the Atrioverter. For each of the staristical analyses used in this study, the unit of anal$y$ sis is an episode rather than a patient. "This is because, in general, each patient has a multiple number of episodes and the actual number of episodes between patients waries, An efficient mechanism for examining this type of data utilizes all observations within a patient and allows berween parient variability in the estimation used for statistical testing. The methods utilized in this study provide for these considerations.

A random effects mixed modeling technique allows for a variable number of repeated observations within patients and takes no consideration the betweenpatient variability. The mixed models random effects technique applied to these data firs a linear function to the relationship of episode length versus time since implant with the slope of this linear function serving as a random effect. In essence, the slope of a given patient's linear relationship is determined individually and the testing of 
the population slope takes into account the variability between each of the individual patient slopes for their individual relationships of the episode duration and time since implant. Since we have multiple observations within patient the variability associated with the estimated slope parameter due to patient can be determined. The statistical test to determine if the slope differs significantly from zero (0), or equivalently if the mean response significantly differ over time, therefore, allows for any inter-patient variability.

For the analysis of the risk of experiencing AF episodes, a multivariate survival analysis described by Prentice was used to compare the hazard or risk of experiencing an episode of $\mathrm{AF}$ as a function of time from implant. This technique allows for the possibility of multiple events within patients as well as the inclusion of the censored time period from the last AF episode until the end of parient follow-up. For all analyses, a p-value of $<0.05$ conferred statistical significance.

\section{RESULTS}

\section{PATIENTS CHARACTERISTICS}

The clinical characteristics of the 51 patients are given in Table 1. On average, 3.9 antiarrhythmic drugs were tried prior to implantation of the device; this includes drugs that were not tolerated and discontinued in addition to drugs that were only partially effective and continued. At implant and at the end of the study, 8 and 3 patients, respectively, were not treated with antiarrhythmic medication. During the study period, 26 of 51 patients $(51 \%)$ underwent changes in their antiarrhythmic drug regimen. At the end of the study, 32 patients were treated with Class III antiarrhythmic agents, singly or in combination with Class I and/or rate control agents. Antiatrhythmic treatment at the beginning and at the end of the study period is shown in Table 2.

Before implantation, 34 patients $(67 \%)$ were on warfarin or heparin therapy, 9 $(18 \%)$ were treated with aspirin and $8(16 \%)$ were not on anricoagulation therapy. During the course of the study, 11 patients $(22 \%)$ had changes in their anticoagulation therapy. One patient discontinued warfarin therapy, five patients were changed from warfarin to aspirin, and five patients were started on warfarin. At the end of the study, $13 \%$ of patients were not taking any form of anticoagulation and an additional $23 \%$ were taking only aspirin. No thromboembolic events occurred during the course of the study. 
Table 1. - Chinical charackeristics of the 51 studied patienrs.

\begin{tabular}{ll}
\hline Meri / Wonten & $40 / 11$ \\
Age (years) & $58 \pm 9(31-77)$ \\
NYHA functional class & \\
I & 36 \\
II & 15 \\
Cardiac disease & \\
$\quad$ Coronary artery disease & 4 \\
$\quad$ Corrected congenitat heart disease & 2 \\
$\quad$ Structural normal heart & 45 \\
LVEF(\%) & $58 \pm 11(38-87)$ \\
LA size (cm) & $4.4 \pm 0.8(3.3-6.6)$
\end{tabular}

LA: left atrium; LVEF: left ventricular ejection fraction. Data presented are number of parients or mean value \pm $S D$ and range.

Table 2.- Antiarnaythmic drugs at implant and end of the srudy period.

\begin{tabular}{lll}
\hline Antiartlyythmic drug & Implant & End of study \\
Single Class I agent & 7 & 8 \\
Class I + Rate control & 3 & 2 \\
Class I + Class III & 4 & 4 \\
Class I + Class III + Rate control & 0 & 3 \\
Single Class III agent & 17 & 15 \\
Combination Class III agents & 0 & 1 \\
Class III + Rate control & 6 & 9 \\
Rate control only & 6 & 6 \\
None & 8 & 3 \\
Number of antiarthythmic drugs / patient & $1.1 \pm 0.7(0-3)$ & $1.4 \pm 0.8(0-3)$ \\
\hline
\end{tabular}

Dana presented are number of paricnts or mean value \pm SD and ange.

\section{FREQUENCY AND DURATION OF AF EPISODES IN PATIENTS WITH THE ATRTOVERTER}

During a follow-up of $260 \pm 144$ days, 45 of the 51 patients had 1230 episodes of AF recorded in the device memory. Five patients had no episodes recorded during a mean follow-up of $164 \pm 112$ days and 1 parient was excluded due to known AF detection problems. In 41 patients, 231 episodes were treated with the device, including the episodes from the patient with $\mathrm{AF}$ detection problems. Five patients had episodes in their device memory but received treatment for none of them.

Of the 1230 episodes, 1161 episodes occurred during vallid monitoring periods (Table 3). The average recurrence rate was $3.9 \pm 5.0$ episodes per patient-month. Of the 231 treated episodes, 190 episodes occurred during valid monitoring periods in 
Table 3. - Distribution of atrial fibrillation episodes th the 45 pacients according to the percentage of treated episodes that occurred during a valid monitoring period.

\begin{tabular}{|c|c|c|c|}
\hline $\begin{array}{l}\text { \% of rreated AF episodes occurring during } \\
\text { a valid monitoring period }\end{array}$ & Nb of Prs & Treated & Nb of AF episodes \\
\hline \multirow[t]{2}{*}{$0 \%$} & 6 & $\mathrm{~N}$ & 37 \\
\hline & & Y & 0 \\
\hline \multirow[t]{2}{*}{$1-10 \%$} & 13 & $\mathbf{N}$ & 803 \\
\hline & & $Y^{\prime \prime}$ & 32 \\
\hline \multirow[t]{2}{*}{$11-40 \%$} & 6 & $\mathrm{~N}$ & 74 \\
\hline & & $\mathrm{Y}$ & 27 \\
\hline \multirow[t]{2}{*}{$41-60 \%$} & 5 & $\mathbb{N}$ & 32 \\
\hline & & $\mathrm{Y}$ & 26 \\
\hline \multirow[t]{2}{*}{$61-90 \%$} & 7 & $\mathbb{N}$ & 24 \\
\hline & & Y & 65 \\
\hline \multirow[t]{2}{*}{$91-100 \%$} & 8 & $N$ & 1 \\
\hline & & $\mathrm{Y}$ & 40 \\
\hline
\end{tabular}

AF: artial fibrillation; $N$ : nontreated AF episode; Nb: number; Pts: partients; $Y$ : rreated AF episode,

39 of 45 patients. Six patients had no treated episodes during valid monitoring periods. This includes the 5 patients that had no treated episodes at all and 1 patient who had only treated episodes that occurred during non-valid monitoring periods.

The number and duration of the treated and nontreated AF episodes according to time since implant is shown in Table 4 . The number of nontreated episodes did not change, however, the number of treated episodes decreased. The median duration of the treated episodes was 17.6 hours and was 3 hours for the nontreated episodes (mean $38 \pm 44$ hours vs $10 \pm 18$ hours, $p<0.05$ ). Additionally, $78 \%$ of the nontreated episodes were less than 8 hours in length as compared to only $28 \%$ of treated episodes.

When the distribution of the AF episodes that occurred during valid monitoring periods was analyzed according to the percentage of episodes treated, the following observations were made (Table 3). In 15 out of the 45 parients (33\%), $81 \%$ of the AF episodes were treated, and in 8 of these patients, the AF episodes were always treated. The AF episodes occurring within a valid monitoring period from 19 patients ( $42 \%$ ) were either not treated at all ( 6 patients with 37 episodes) or less than $10 \%$ of episodes were treated (13 patients with 835 episodes).

\section{EFFECT OF ATRIOVERTER THERAPY ON THE INTERVAL BETWEEN SPONTANEOUS EPISODES OF AF}

To evaluate the time course of symptomatic, treated AF episode frequency, we evaluated the duration between treated AF episodes and the risk of having an episode as 
Table 4. - Number and duration of the treated and nontreated atriall fibrillarion episodes occurring during valid monitoring periods according to time since implant.

\begin{tabular}{|c|c|c|c|c|c|c|}
\hline \multirow{3}{*}{ Days since implant } & \multicolumn{3}{|c|}{ Nontreared episodes } & \multicolumn{3}{|c|}{ Treated episodes } \\
\hline & \multirow[t]{2}{*}{ N } & \multicolumn{2}{|c|}{ Duration (hours) } & \multirow[t]{2}{*}{ N } & \multicolumn{2}{|c|}{ Duration (hours) } \\
\hline & & mean $\pm S D$ & median & & mean $4 \mathrm{SD}$ & median \\
\hline $0-50$ & 213 & $7.5 \pm 15.7$ & 2.0 & 93 & $38.9 \pm 42.5$ & 22.4 \\
\hline $51-125$ & 261 & $11.6 \pm 20.3$ & 3.0 & 48 & $34.0 \pm 44.6$ & 15.5 \\
\hline $126 \cdot 200$ & 224 & $8.2 \pm 19.1$ & 20 & 33 & $38.5 \pm 47.8$ & 13.6 \\
\hline$>200$ & 273 & $12.1 \pm 15.0$ & 4.0 & 16 & $43.7 \pm 47.5$ & 20.3 \\
\hline
\end{tabular}

N: number of arrial fibrillation episodes.

time since implant increased. For all these analyses, patient's follow-up intervals were measured relarive to the day of implant. The result of the first mixed models analysis is shown in Figure 1. The goal of this exploratory analysis was to examine the mean length of time during which no AF is observed categorized by the number of days since implant that the next treated episode occurs. Only patients with 2 or more spontaneous episodes for which therapy with the Atriowerter was sought were included ( $\mathrm{n}=33$ patients). Additionally, we only considered intervals between episodes where the actual time was observed, i.e., excluded from the analysis is the time from the last episode in a patient to the end of the study. From this figure and the predicted line there appears to be a trend in the mean time between treated episodes and time since implant ( $\mathrm{p}<0.05)$. Namely, as time since implant increases the mean interval between treated episodes also increases.

A graphical evaluation of the change of the risk of having an episode requiring Atrioverter treatment is shown in Figure 2. For this, all patients that had at least 1 treated episode were included ( $n=4 \mathbb{1}$ patients). For a given time period since implant, the number of parients at risk for an episode was determined, i.e., the number of patients actively enrolled in the study. The estimated risk was then calculated as the ratio of the number of parients which had at least 1 treated episode during the given time period over the number of patients at risk. As can be seen in Figure 2, the risk of observing an episode is decreasing as rime since implant increases.

Lastly, for subjects that had at least 1 episode $(\mathrm{n}=41$ ), a multivariate survival analysis was performed to examine the hazard or risk of experiencing an AF episode as time since implant increased. In general, this technique stratifies the data by episode number where the response is the time between treated episodes and the number of days since implant is considered as a possible explanatory variable. This analysis then tests over strata if the risk of experiencing an episode depends on the number of days postimplant. It was shown that the hazard of experiencing an episode decreases as time from implant increases $(p<0.05)$ (Table 5). Based on this analysis, the risk of experiencing an episode of AF, requiring defibrillator therapy, 60 


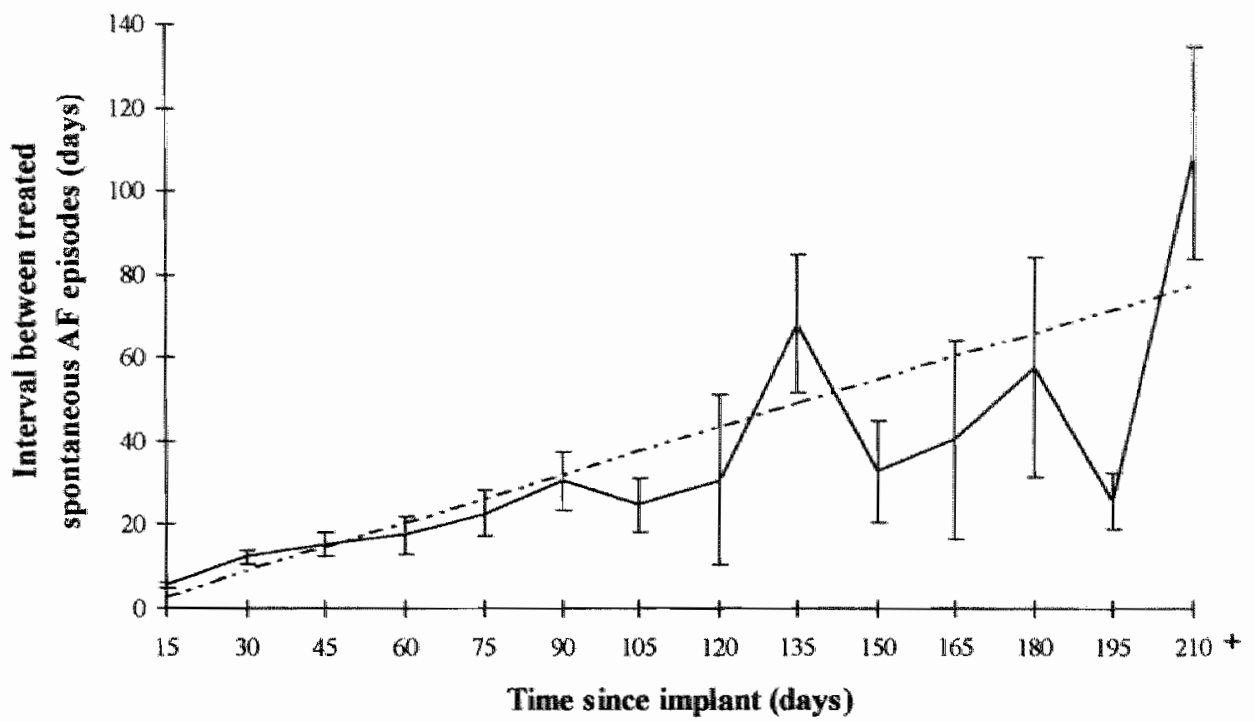

Figure 1. - This figure shows an increase of the mean interval between treated spontaneous atrial fibrillation episodes as time since implantation of the Atrioverter increase. Solid curve = the observed mean and standard error of the interval berween treated spontaneous atrial fibrillation episodes. Dashed curve $=$ the predicted mean of the interval berween treated spontaneous atrial fibrillation episodes. $A F=$ atrial fibrillation.

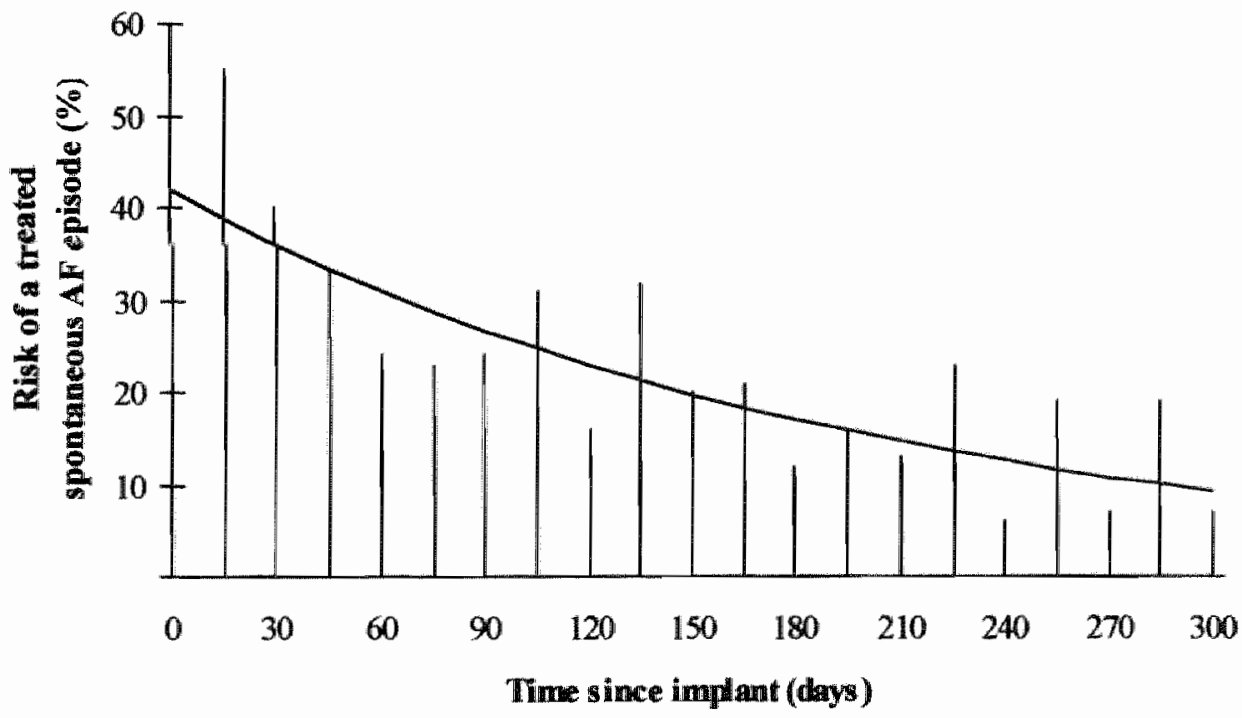

Figure 2. - Logarithmic function between the risk of a treated spontancous atrial fibrillation episode and the tine since implantation of the Arrioverter. As time since implantation increases, the risk of a treared spontaneous atrial fibrillarion episode decreases. AF = atrial fibrillation. 
Table 5. - Reduction in the risk of a treated arrial fibrillation episode relative to day 7 after Amioverter implantation.

\begin{tabular}{cl}
\hline Days since implant & Reduction in risk \\
\hline 14 & $16.7 \%$ \\
30 & $44.5 \%$ \\
60 & $73.1 \%$ \\
90 & $86.3 \%$ \\
120 & $92.6 \%$ \\
\hline
\end{tabular}

days after implant was reduced by $73 \%$ as compared to the risk of experiencing an episode of $\mathrm{AF}^{7}$ days after implant.

One other aspect that we evaluated was whether the nontreated episodes had any impact on the prolongation of the time between treated episodes of AF and wherher this was due to the parient being reluctant to have Atrioverter therapy. In order to explore this, the duration of the treated and nontreated episodes were compared as time increased from implant. The basis for the use of this comparison is that since the treared episodes were of longer duration (as mentioned previously) and if potentially treatable, longer duration episodes went untreated based on the patients reluctance to Atrioverter therapy, the mean and median duration of the nontreated episodes would increase over time. We found that there was no increase in the duration of the nontreated episodes over time $(p>0.05)$. In fact, the number of nontreated episodes did not increase nor diminish over time ( $p>0.05$ ).

\section{DISCUSSION}

\section{NATURAL HISTORY OF AF}

The paroxysmal nature of AF in this patient population is demonstrated by the presence of multiple episodes of the arrhythmia during the 20 month follow-up period. Although only patients with estimated arrhythmia recurrence intervals between one week and three months were eligible for inclusion, 5 of the 51 patients had no episodes of AF during a mean follow-up duration of $164 \pm 112$ days. This may be explained by the natural history of the arrhythmia in these patients. Additionally, none of the patients who had the device implanted at the time of the end of the study had progressed to chronic, permanent AF.

Forty-five patients had more than 1000 spontaneous AF episodes during the follow-up period. In patients with both treated and nontreated episodes, treated episodes had a significantly longer duration than the nontreated ones. This is partially due to the fact that patients had to return to the hospital for treatment often requiring more than one hour of transportation or that these nontreated episodes 
may have been asymptomatic. When the spontaneous AF episodes wete divided based on 8 hours duration, it was observed that $72 \%$ of the nontreated episodes were less than 8 hours while $78 \%$ of the treated episodes were longer than 8 hours duration. These data suggest that there are patients that have both episodes of long duration requiring treatment as well as short or asymptomatic episodes that are well tolerated or may go ignored. Whether these short-lasting, nontreated episodes affect the need for anticoagulation in the Arrioverter patient remains unknown. Most patients in this study were anticoagulated and no thromboembolic events were seen despite these nontreated episodes and therefore, no conclusions can be drawn. However, when patients are able to treat episodes with the Atrioverter outside the hospital, more of the shorter duration episodes may be treated and therefore, may have an impact on the need for anticoagulation.

\section{REVERSION OF CHRONIC CHANGES}

An experimental study in chronically instrumented goats has shown that AF induces changes in the electrophysiologic properties of the atria which may lead to the development of chronic AF (electrical remodeling)7. This animal study suggested to interrupt the arrhythmia as soon as possible in order to prevent subsequent AF episodes. The electrical atrial remodeling completely reverted within 1 week after restoration of sinus rhythm in goats. The question which can be asked of the data from the present study is whether the electrical atrial remodeling has changed (reflected by a decrease in the number of AF episodes) after repeated intraatrial defibrillation of our patients with a long history of AF. If we take both treated and nontreated AF episodes into consideration, it seems that no reversion of the atrial electrical remodeling has occurred at this point of follow-up. However, one must also consider that a majority of these nontreated episodes (803 episodes) were from only 13 patients, potentially skewing these data. When only the treated episodes (190 episodes) were analyzed, the mean interval berween these $\mathrm{AF}$ episodes increased as time from implantation increased, potentially denoting some form of reversion of electrical remodeling. The unchanged frequency of nontreated episodes demonstrates that the increase in time between treated episodes cannot be fully explained by the patients reluctance to Atrioverter therapy. The reason why the number of the nontreated or short-lasting episodes did not decrease over time is not yet understood. Perhaps, with the ambulatory use of the device in either an automatic mode with defibrillation after a fixed time from episode onset or a parient activated mode, these short-lasting episodes will also be treated and decrease over time. 
Antiarthythmic drugs could have played a role in affecting the number, frequency and duration of $\mathrm{AF}$ episodes after implantation of the device. As the use of these agents was not controlled in this study, no evaluation of their effect on possible reversibility of remodeling could be made from these episode data. Future studies will be needed to separate the relative contribution of the antiarrhythmic drug use and repeated defibrillation therapy.

Symptoms were not collected from the patients during the study. Therefore, it could not be determined whether the nontreated episodes were asymptomatic and what the time of onset of symptoms to device treatment was.

\section{CONCLUSIONS}

In patients wirh symptomatic, recurrent, drug-refractory AF, the frequency of longlasting episodes, which were treated with repeated defibrillation using the Atrioverter, decreased. The number and duration of short-lasting, nontreated episodes did not change during the 20 month study period. Prompt termination of all episodes of AF using out of hospital trearment with the Atrioverter needs to be evaluated.

\section{ACKNOWLEDGEMENT}

We acknowledge the efforts of Kathryn S. Dawson, Ph.D., for the statistical analysis presented herein.

\section{REFERENCES}

1. Murgatroyd PD, Shade AKB, Sopher SM, er al. Efficacy and tolerability of transwenous low energy cardioversion of paroxysmal arrial fibrillarion in humans. J Am Coll Cardiol 1995;25:1347-53.

2. Schmit $\mathrm{C}$. Alt E, Plewan A, et al. Low energy intracardiac cardiowersion after faled conventional external cardioversion of atrial fibrillation. I Am Coll Cardiol 1996;28:994-9.

3. Lévy $\$$, Ricard P, Lau CP, et al. Multicenter low energy transwenous atrial defibrillation (XAD) trial results in different subsets of atrial fibrillation. J Am Coll Cardiol 1997;29:750-5.

4. Wellens HJj, Lau CP, Lüderitz B, et al. The Arnoverter, an implantable device for the trearment of atrial fibrillarion. Circulation 1998:98:1651-6.

5. Tse HF, Lau CP, Sta JS, et all. Atrial fibrillation detection and R-wave synchronization by Metrix implantable atrial defibrillator. Implications for long-rerm efficacy and safery. Circularion $1999 ; 99: 1446-51$. 
6. Prentice RL, Willians BJ, Peterson AV. On the regression analyas of nultivariate falure time dara. Biometrika 1981;68:373-9.

7. Wijfels MCEF, Kirchhof CJHI, Dorland R. Allessie MA. Atrial fibrillation begets arrial fibrillation. A study in awake chronically instrumented goars. Circulation 1995;92:1954-68.

\section{APPENDIX}

\section{LIST OF PARTICIPATING CENTERS AND INVESTIGATORS}

Academic Hospüral Gent, Gent, Belgium (L. Jordaens, R. Tavemier, F. Provenier); Hopital Nond Marseille, Marseille, France (S. Levy, W. Taramasco, E. Dolla) i Hopital Cardiologique, Lille, France (S. Kacet, D. Lacroix, P. le Franc, D. Klug, C. Kouakam); Hôpital "Hôtel Dieu", Rennes, France (C. Daubert, D. Gras, P. Mabo, D. Pavin); Universiry of Bonn, Bonn, Germany (B. Luderirz, W. Jung); Klinikum der Stadt Ludwigshafen, Ludwigshafen, Germany ( $K$. Seidl); Acadenic Hospiral Maastrichr, Maastricht, the Netherlands (H. Wellens, C. Timmermans, L.M. Rodriguex); Academic Hospiral Groningen, Groningen, the Netherlands (H. Crijns, I. van Gelder); Sahigrenska Sjukhuset, Göteburg, Sweden (N. Edvardsson, I. Lurje, M. Aumes, H. Walfridsson); Karolinska Hospital, Stockholm, Sweden (M. Rosenqvist, C. Linde); St. George's Hospital, London, United Kingdom (A.J. Camm, E. Rowland, D. Ward, S.M. Sopher, M. Gallagher, A. Slade, J. Waktare); The Queen Mary Hospital, University of Hong Kong, China, (C.P. Lau, H.F. Tse, N.S. Lok, K. Lee); University of Calgary, Calgary, Canada (D.G. Wyse); Institute of Cardiology, Montréal, Canada (B. Thibaut, M. Talaijic); St. Michael's Hospital, Toronto, Canada (P. Dorian, D. Newman); St. Luke's Medical Center, Milwaukee, Wis, USA (J. Sra. M. Akhtar, M. Miehl, Z. Blanck, S. Deshpande, A. Dhala, H. Jazayeri, D. Kress); University Hospitals of Cleveland, Cleveland, Ohio, USA (L. Biblo, J. Mackall, A. Waldo, D. Rosenbaum, M. Carison); University Hospital, Ann Arbor, Mich, USA (A. Strickberger, F. Morady, E. Daoud, R. Goyal, M. Harvey, K. Ching Man); and University of Utah Medical Center, Salt Latke City, Utalt, USA (R. Freedman, R. Klein, J. Mason, G. Muelheims, P. Spector, S. Compron). 

CHAPTER 8

\section{Are electrophysiological changes induced by longer lasting atrial fibrillation reversible? Observations using the atrial defibrillator}

Luz-Maria Rodriguez, MD; Carl Timmermans, MD; Hein J] Wellens, MD

From the Department of Cardiollogy, Academic Hospital, Maastricht, the Netherlands

Abstract presented at the $77^{\text {th }}$ Annual Scientific Session of the American Heart Accaciation 1998 (Circulation 1998:98:1-713)

Pablished in Circulation 1999;100:113-116 


\section{A BSTRACT}

Background. Studies in animal hearts have shown shortening of the atrial effective refractory period (AERP) and loss of the relation between the AERP and heart rate after prolonged periods of atrial fibrillation (AF). The purposes of this study were (1) to evaluate atrial electrophysiology after a long period of sinus rhythm in patients who had longer lasting recurrent AF that was successfully treated with the Merrix Atrioverter and (2) to analyze the effect of prompt cardioversion on subsequent AF episodes and the duration of sinus rhythm.

Methods and Restlts. Four patients with recurrent AF (duration, 3 to 21 years; mean $\pm S D, 13 \pm 7.6$ years) were studied after the implantation of an Arrioverter. The Atrioverter stores and analyzes 3 minutes of cardiac rhythm every hour. Before implantation, AERP was measured. During a mean follow-up of 14 months, 52 spontaneous (39 treated and 18 nontreated) AF episodes occurred while the patients were on antiarthythmic drugs. All patients were electrophysiologically studied after they had been in sinus rhythm for at least 1000 hours (range, 1052 to 2675 hours). Before Atrioverter implantation, AF was induced by 1 atrial premature beat in 3 patients and not induced in the remaining patient. After a long period in sinus rhythm ( $>1000$ hours), AF could be induced in the same 3 patients in the same way as before implantation. In the parient in whom no AF was induced, right AERP values measured using the single extrastimulus technique at 3 pacing cycle lengths $(600,500$, and $430 \mathrm{~ms})$ were similar to those before implantation.

Conclusions. AF was still inducible by a single atrial premature beat after long episodes of sinus rhythm in 3 of 4 patients wirh previously longer lasting $A F$. In the patient in whom no AF was induced, AERP behaved like it did before implantation. In these patients with longer lasting recurrent AF no return to "normal" atrial electrophysiology could be demonstrated. 
Studies in the goat showed shortening of the atrial effective refractory period (AERP) and loss of the relation between the AERP duration and heart rate after prolonged periods of atrial fibrillation (AF) (remodeling). Furthermore, AF was more difficult to reinduce as time after conversion of an $\mathrm{AF}$ episode increased ${ }^{1}$. Those experiments suggested that the findings on atrial electrophysiology after long periods of AF were the result rather than the cause ${ }^{2}$ of AF. They also argued for prompt termination of AF using conventional or new ${ }^{3}$ modalities to reverse the electrophysiological changes favoring the development of chronic AF. The purposes of this study were (1) to evaluate the electrophysiological properties of the atrium after a long period of sinus thythm in patients who previously had long-lasting recurrent AF that was promptly and successfully treated with the Metrix Atrioverter and (2) to analyze the effect of prompt cardioversion on subsequent AF episodes and the duration of sinus rhythm.

\section{METHODS}

\section{PATIENTS}

Until May of 1998, 13 parients with recurrent, drug-refractory AF received the Metrix Atrioverter at our institution. The 4 patients with the longest follow-up are the subject of this study. Their clinical characteristics are given in the Table. The mean duration of the arrhythmia before Atrioverter implantation was $13 \pm 7.6$ years.

Table - Clinscal characteristics of the patients studied.

\begin{tabular}{lllllll}
\hline Pr & Sex & Age (years) & \multicolumn{2}{c}{ LA size $(\mathrm{mm})$} & Etiology & Failed AAD (median) \\
\hline 1 & M & 51 & 47 & 41 & Lone & 4 \\
2 & F & 65 & 46 & 45 & Lone & 3 \\
3 & M & 65 & 58 & 56 & CAD & 4 \\
4 & M & 66 & 53 & 66 & Lone & 4 \\
\hline
\end{tabular}

AAD: antiarthythmic drug; AF: arrial fibrillation; Afrer: after implant; Before: before implant; CAD: coronary arrery disease; female; L.A: left atrium; M: male; Pr: patient.

\section{ELECTROPHYSIOLOGICAL STUDY}

All parients underwent internal atrial defibrillation before implantation. This technique has been described elsewhere ${ }^{4}$. Except for amiodarone, antiarrhythmic drugs (AAD) were discontinued for at least 5 half-lives before the electrophysiologic study. After 10 minutes of stable sinus rhythm, electrophysiological examination was 
performed. In addition, a quadripolar catheter was positioned in the high righe atrium to measure the AERP. Electrodes 1 and 2 were used for pacing and electrodes 3 and 4 to record atrial activity. The AERP was defined as the longest $S_{1}-S_{2}$ coupling interval failing atrial capture. The mean atrial pacing threshold was $1 \pm 0.3 \mathrm{~mA}$. Pacing was performed at rwice threshold. After a 10-beat drive, progressively decremental (10 ms), atrial extrastimuli were delivered, beginning at a coupling interval $\left(S_{1}-S_{2}\right)$ of $360 \mathrm{~ms}$, using drive cycle lengths of 600,500 , and $430 \mathrm{~ms}$, until the AERP was reached or AF was induced. The technique for implanting the Metrix Atrioverter has been described elsewhere ${ }^{4}$. After implantation, the device was programmed in monitoring mode to evaluate cardiac rhythm every hour for 3 minutes, with data logged into memory each time AF was derected. Patients were asked to come to the hospital for prompt treatment of their spontaneous episodes of AF. The electrophysiologic study was repeated after patients were in sinus rhythm for at least 1000 hours (range, 1052 to 2675 hours) while on AAD. For the 2 years before implantation, vigorous attempts were made to document all AF episodes. The study protocol was approved by the Human Research Committee of our Institution.

\section{RESULTS}

\section{ATRIAL FIBRILLATION EPISODES BEFORE IMPLANTATION}

Patient 1 had 1 AF episode every month that lasted from hours to 5 days. During the last 2 years, these episodes required external cardioversion to achieve sinus rhythm. Parient 2 experienced daily AF episodes lasting from minutes to hours. Patient 3 had in the last 2 years, $1 \mathrm{AF}$ episode per month lasting from hours to 1 week. Conversion to sinus rhythm could only be obtained with external cardioversion. Finally, patient 4 experienced 1 AF episode every 3 months. These episodes lasted 3 days and required external cardioversion.

\section{ELECTROPHYSIOLOGIC FINDINGS BEFORE IMPLANTATION}

Before implantation, sustained ( $>30 \mathrm{~min}$ ) AF was easily induced with 1 atrial premature beat $(\mathrm{APB})$ in 3 patients. In patient $1 \mathrm{AF}$ was induced with a drive cycle length of $600 \mathrm{~ms}$ and $S_{1}-S_{2}$ of $260 \mathrm{~ms}$. In patient $2, A F$ was induced with a drive cycle length of $600 \mathrm{~ms}$ and a $S_{1}-S_{2}$ of $280 \mathrm{~ms}$, and in patient 4 , with a drive cycle length of $500 \mathrm{~ms}$ and a $S_{1}-S_{2}$ of $280 \mathrm{~ms}$. In patient 3 . AF could not be induced using 3 different pacing cycle lengths and up to 2 atrial extrastimuli. The AERPs measured in the high right atrium with a drive cycle length of 600,500 , and $430 \mathrm{~ms}$ were 200 , 220 , and $200 \mathrm{~ms}$, respectively. 
In a follow-up period of 597 days, patient 1 had 7 treated and 2 nontreated (self-terminating) spontaneous episodes of AF. The mean duration of the treated episodes was $13.06 \pm 8.36$ hours (range, 7 to 33 hours), and the durations of the 2 nontreated episodes were 7 and 8 hours, respectively. Patient 2 had 14 treated and 10 nontreated episodes of AF in a follow-up of 365 days. The mean duration of the treated episodes was $5.53 \pm 3.38$ hours (range, 3.3 to 1.0 hours) and of the nontreated episodes, $5.30 \pm 3.77$ hours ( 0.5 to 14.3 hours). Patient 3 had 15 treated and 6 nontreated episodes of $A F$ in a period of 401 days. The mean duration of the treated episodes was $10.39 \pm 4.61$ hours (range, 4 to 17.3 hours). All 6 nontreated AF episodes lasted less than 1 hour. Finally, parient 4 had only 3 AF episodes (all treated) within a period of 288 days. The mean duration of these episodes was $31.57 \pm 31.0$ hours (range, 10.5 to 107 hours). The reason why this parient had longer durations of AF episodes than the other 3 patients was because the patient was less symptomatic.

During the course of this study, the AAD regimen was unchanged in patients 1, 3 , and 4, whereas in patient 2, sotalol was added to flecainide. Thus, the current medication consisted of amiodarone in patient 1 , sotalol and flecainide in patient 2 , flecainide and metoprolol in patient 3 , and amiodarone in patient 4.

\section{ELECTROPHYSIOLOGIC FINDINGS AFTER A LONG PERIOD OF SINUS RHYTHM}

Sustained ( $>30 \mathrm{~min}$ ) AF was induced in patient 1 after 1052 hours (43.8 days) of sinus rhythm. AF was induced at a drive cycle length of 500 ms and a $S_{1}-S_{2}$ of 300 ms. This episode was terminated with a 3-J shock from the Atrioverter. In patient 2, sustained AF was induced at a drive cycle length of $600 \mathrm{~ms}$ and a $S_{1}-S_{2}$ of $230 \mathrm{~ms}$ after being in sinus rhythm for a period of 2675 hours (111.46 days). This episode was converted to sinus rhythm with $150 \mathrm{mg}$ of flecainide intravenously. In patient 3 , AF could not be induced by atrial pacing using 3 different pacing cycle lengths and up to 2 APBs after a period of sinus rhythm of 1075 hours ( 44.79 days). The AERP values measured in the high right atrium at drive cycle lengths of 600,500 , and 430 $\mathrm{ms}$ were 250,240 , and $250 \mathrm{~ms}$, respectively. Finally, in patient 4 , AF was induced at a drive cycle length of $500 \mathrm{~ms}$ and a single APB of $240 \mathrm{msec}$ afrer 2374 hours $(98.9$ days) of sinus rhythm. In this parient, stable sinus rhythm was obtained after 2 Atrioverter shocks of $6 \mathrm{~J}$ and the intravenous administration of $150 \mathrm{mg}$ of flecainide.

\section{EFFECT OF INTERNAL ATRIAL DEFIBRILLATION ON AF AND SINUS RHYTHM DURATION}

The effect of repeated internal atrial defibrillation on the duration of AF and sinus rhythm duration is shown in the Figure. Only in patient 2 did the duration of the 
AF episodes seem to shorten and the duration of sinus rhythm to prolong as the time from implantation increased. The duration of sinus rhythm started to prolong 120 days after implantation. In the remaining 3 patients, the effect of repeated cardioversion on the prolongation of the duration of sinus rhythm and the shortening of the duration of AF episodes did not (yet?) occur.

\section{DISCUSSION}

To our knowledge, this is the first study prospectively reporting on atrial electrophysiology after a long period of sinus rhythm ( $>40$ days) in patients with recurrent $\mathrm{AF}$ treated with the Atrioverter. It shows thar in patients with recurrent $\mathrm{AF}$, despite the fact that the atria have been in sinus rhythm for a long period, sustained $\mathrm{AF}$ ( $>30$ minutes) was easily induced with $1 \mathrm{APB}$ in 3 patients. In the remaining patient in whom no $A F$ could be induced, the lack of rate-dependent behavior of the AERP remained unchanged. Repetitive internal atrial defibrillation resulted in shortening of the duration of the AF episodes and prolongation of the duration of sinus rhythm in only 1 patient. Interestingly, in that patient, AF was induced with a single APB after sinus rhythm had been present for 111 days. These findings show that in the follow-up period, no change occurred in atrial electrophysiology in our 4 patients. In goats with induced chronic AF, changes in atrial electrophysiology completely reverted after 1 week of sinus rhythm. Moreover, the inducibility of AF became more difficult after 1 week of sinus rhythm ${ }^{\text {l }}$.

In induced $A F$ episodes in the human heart, ${ }^{5,6}$ without documentation of spontaneous AF, it was shown that the AERP after AF returned to preAF measurements after a mean of $8^{5}$ or $13^{6}$ minutes. Furthermore, in those studies, induction of a second AF episode became more difficult as time elapsed from spontaneous conversion; it was $0 \%$ at 10 minutes. 5

At the time of this second electrophysiologic study of our patients on AADs, AF was still inducible in 3 patients. In the patient in whom AF was not inducible, the AERP had increased slightly.

Our study does not question the value of the implantable atrial defibrillator to rapidly convert atrial fibrillation to sinus rhythm, but the observations in our 4 patients with longer lasting recurrent Af showed that atrial electrophysiology did not return to normal. If the nontreated episodes ( $\leq 5$ hours) were responsible for this unchanged atrial electrophysiology, it would imply that all shorter episodes need to be treated promptly to obtain complete restoration of atrial electrophysiology. It may be more likely, however, that after a long period of AF recurrences, morphological, genetic, and electrophysiologic changes occur that prevent atrial electrophysiology from returning to normal. It is clear that the type of AF in our patients differed from the first episode of longer lasting AF studied in goats. ${ }^{1}$ The possibility of reversing the arrial elecrrophysiological changes in patients after the 

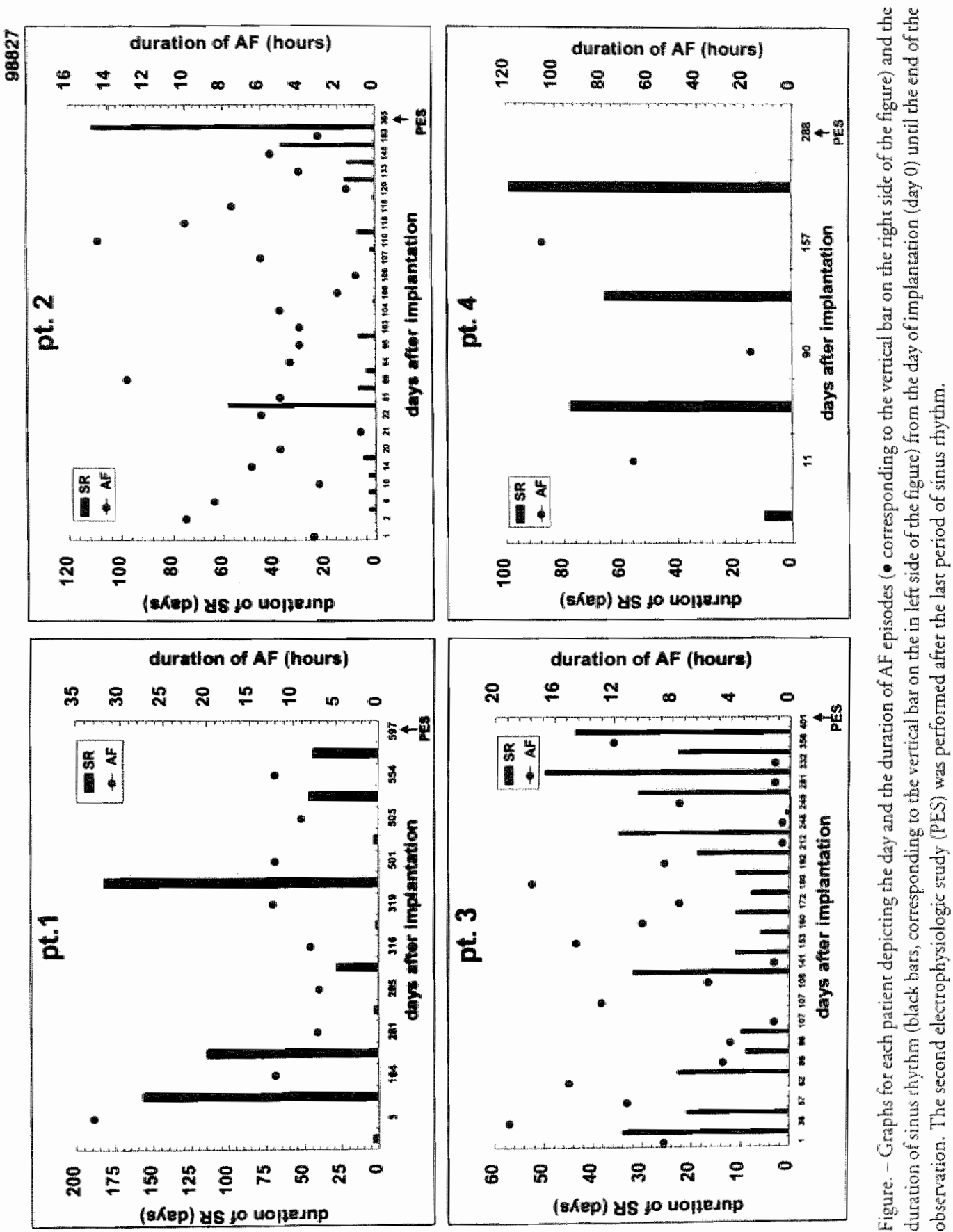
conversion of their first few episodes of AF must be investigated. More information is needed about the role of the number and duration of AF episodes on the reversibiliry of changes in atrial electrophysiology.

\section{LIMITATIONS OF THE STUDY}

The small number of patients included in our study is a limitation. Furthermore, the Arrioverter was programmed to detect AF every 60 minutes. Some short-lasting AF episodes occurring between 2 scanning intervals were perhaps not detected by the device.

\section{CONCLUSION}

In 4 patients with a long standing history of recurrent AF who were treated with the implantable Atrioverter, AF was easily reinduced by a single APB after a long episode. of sinus rhythm in 3 patients. In the patient in whom $A F$ was not induced, the AERP had not changed compared with values before Atrioverter implantation. Therefore, in these patients, no return to "normal" atrial electrophysiology could be demonstrated.

\section{REFERENCES}

1. Wijffels MCEF, Kirchhof CJHJ, Dorland R, Allessie MA. Atrial fibrillation begets atrial fibrillation. A study in awake chronically instrumented goats. Circulation. 1995;92:1954-1968

2. Attuel P. Childers RW, Haissaguerre M, Leclerq J, Mujica J, Coumel P. Failure in the rate adaptation of the atrial refractory periods. new parameter to asses atrial vulnerability. Pacing Clin Electrophysiol. 1984;7:1382-1386.

3. Wellens HJ), Lau CP, Lüderitz B, Akhtar M, Waldo AL, Camm AJ, Timnermans C, Tse HF, Jung W, Jordaens L, Ayers $G$., for the Merrix Investigators. The Arrioverter, an implantable device for treatment of atrial fibrillation. Cincuhation. 1998;98:1651-1656.

4. Timmermans $C_{\text {n }}$ Rodriguez LM, Smeets JLRM, Wellens HJJ. Immediate reinitiacion of acrial fibrillation after internal atrial defibrillation. Jonmal Cardioutsc Electrophysiol. 1998:2:122-128.

5. Daoud EG, Bogun F, Goyal R, Harvey M, Man C, Strickberger SA, Morady F. Effect of atrial fibrillation on atrial refractoriness in thumans. Circtation. 1996;94:1600-1606.

6. Yu WC, Chen SA, Lee SH, Tai CT, Feng AN, Kuo BIT, Ding YA, Chang MS. Tachycardiainduced change of atriat refractory period in humans. race dependency and effects of anriarthythmic drugs. Circulation. 1998;97:2331-2337. 
CHPTE

\section{Management of atrial arrhythmias secondary to severe congenital heart disease with the Atrioverter}

Carl Timmermans*, MD; Luz-Maria Rodriguez*, MD; David Reuter**, MD; Jan Stappers*, MD; Paul JC Barenbrug***, MD; Emile C Cheriex*, MD; Hein JJ Wellens*, MD

From the "Deparment of Cardiology and ***Cardiopulmonary Surgery, Academic Hospital Maastricht, Maastricht, The Netherlands; and *'Seartle Children's Hospiral, Searte, WA, USA. 


\section{A B S T R ACT}

An atrial defibrillator was implanted in a patient with congenitally corrected transposition of the great arteries, associated cardiac abnormalities and persistent atrial arrhythmias. During a follow-up of 15 months, 14 of 20 spontaneous episodes of his arrhythmias were successfully treated with the device. Two of these episodes were converted to sinus rhythm during ambulatory use of the device. Successful use of the device required implantation of a third defibrillation lead in the persistent left sided superior caval vein and rigid control of congestive heart failure. An atrial defibrillator may be a valid treatment option in patients with congenital heart disease crippled by atrial fibrillation. 
Improved surgical techniques and better medical management have significantly prolonged the life span of many patients with congenital heart disease. However, the anatomic abnormalities predispose many of these patients to atrial arrhythmias. ${ }^{1}$ The advent of an implantable atrial defibrillator expanded our therapeutic repertoire to treat atrial fibrillation (AF). ${ }^{2}$ We report herein the results of the first patient with congenital heart disease implanted with the Metrix ${ }^{T M}$ Atrioverter (InControl Inc., Redmond, WA).

\section{METHODS}

The parient is a 39-year-old man with a congenitally corrected transposition of the great arteries and several additional cardiac abnormalities. Echocardiography and magnetic resonance imaging revealed both ventriculloarterial discordant connecrions (transposition of the great arteries) and atrioventricular discordant connections, dilated right and left atria, atrial septal defect, tricuspid atresia with minimal residual ostium, hypoplastic right ventricle, ventricular septal defect, cardiomegaly and a persistent left sided superior vena cava. Aortic, pulmonic, mitral and tricuspid insufficiency were also documented.

Prior to device implant, the parient had suffered from persistent AF for 8 years. While in AF his symptoms include fatigue, dizziness, palpitations and dyspnea NYHA class III. His symptoms are markedly improved while in sinus rhythm. The last 2 years, the patient underwent 8 external cardioversions. Physical examination while in AF revealed a cyanotic patient with digital clubbing, a blood pressure of $96 / 60 \mathrm{~mm} \mathrm{Hg}$, a pulse rate of $120 / \mathrm{min}$, an 02 saturation of $87 \%$, a hyperdynamic precordium and a laterally displaced point of maximal cardiac impulse. There was at grade II/IV sysrolic murmur at the lower left sternal border and a single S1. No hepatosplenomegaly was found. Despite various combinations of antiarrhythmic drugs, his arthythmias occurred more frequently and necessitated external cardioversion within 24 hours. No surgical intervention was considered possible. Six months prior to device implant, the patient underwent 3 separate internal cardioversion procedures for AF, 1 of them after a failed external cardioversion. Successful internal atrial defibrillation was consistently obtained with $4.5 \mathrm{~J}(240 \mathrm{~V})$, and sinus rhythm lasted for 94, 15 and 11 days respectively. An electrophysiological study revealed both coarse AF and a multifocal atrial tachycardia originating from the high crista terminalis. An ablation procedure was aborted because of the multifocal nature of the atrial tachycardia. Given the persistent nature of the parient's AF, the poor efficacy of antiarrhythmic drugs, the markedly improved clinical status in sinus rhythm as compared to $\mathrm{AF}$ and the low atrial defibrillation threshold, the decision was made to implant the Atrioverter. 

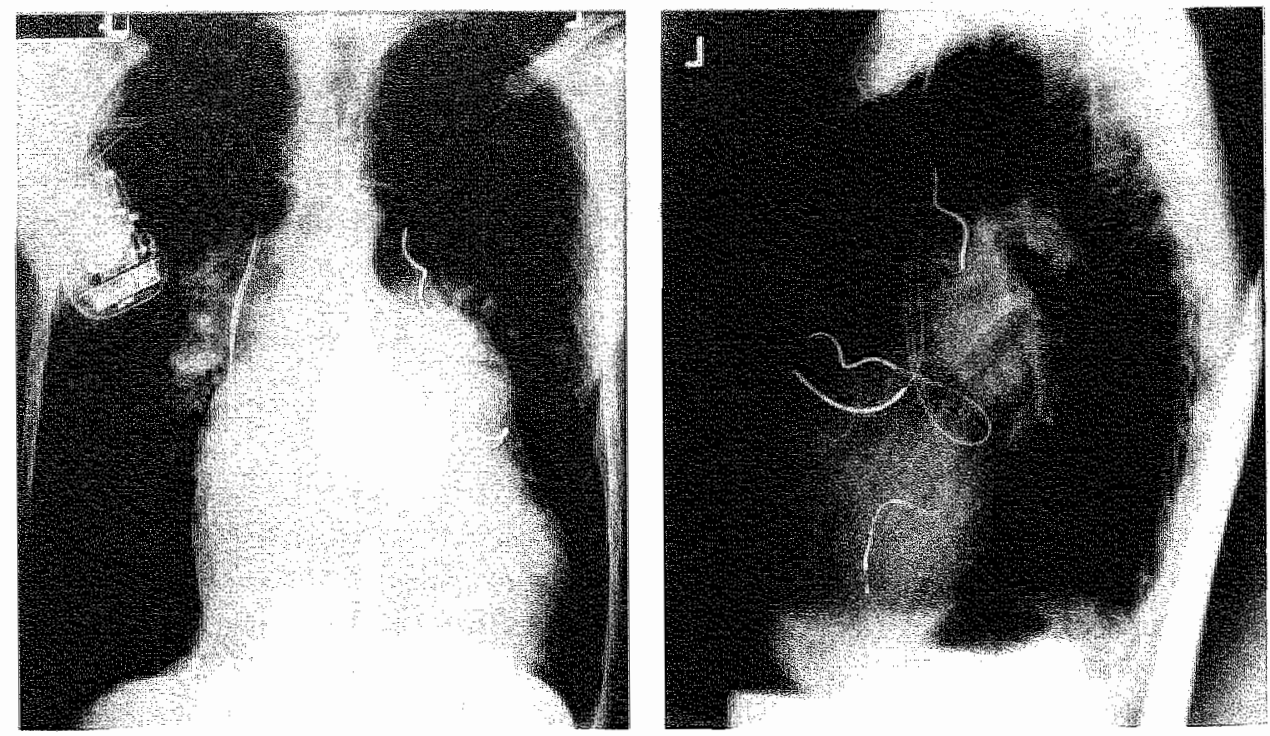

Figure. - Anteroposterior and left lateral chesit x-ray showing a right pectorally implanted Atrioverter with leads in right atrial appendix, coronary sinus, persistent left sided superior vena cava, and morphologic left ventricle.

\section{RESULTS}

After informed consent, the Atrioverter was implanted in November 1997. The right atrial defibrillation lead was acrively fixed in the right atrial appendix. The coronary sinus defibrillation lead was inserted in the coronary sinus and a bipolar ventricular pacing lead was positioned in the morphologic left ventricle (Figure). After implantation the patient was continued on treatment with an ACE inhibitor and coumadine. Amiodarone therapy was interrupted and replaced by flecainide because of subclinical hyporhyroidism. Over the next 5 months, the patient came to the hospital with 11 spontaneous episodes of AF/atrial tachycardia. Only 4 episodes were successfully converted to sustained sinus thythm using the device. In 6 of the episodes, the Atrioverter was unable to achieve sustained sinus rhythm and the parient successfully underwent external cardioversion in the antero-posterior direction. One episode terminated spontaneously after 1 hour. Meanwhile, sotalol ( $2 \times 40$ $\mathrm{mg} / \mathrm{day})$ and thereafter amiodarone $(200 \mathrm{mg} /$ day) were added to his therapy and flecainide was discontinued. Suspecting that the poor efficacy of the Atrioverter over the past months was due to high atrial defibrillation thresholds in the presence of large atria, a third defibrillation lead was placed in the persistent left sided superior vena cava through the innominate vein (Figure) to encircle more atrial tissue, thereby decreasing the atrial defibrillation threshold. Thereafter, 2 spontaneous episodes of AF/atrial tachycardia were successfully treated wirh the Atrioverter. One and a half months after the implantation of the additional lead the parient was 
admirted to the hospital because of congestive heart failure. At the time of hospital admission, internal as well as external cardioversion failed to achieve sustained sinus rhythm. Following 8 days of forced diuresis, he was successfully cardioverred with the Atrioverter. Thereafter, 6 episodes of AF/atrial tachycardia occurred. Three of them ( 2 episodes of 1 hour and 1 episode of 10 hours) terminated spontaneously and the remaining 3 were successfully treated with the device.

Since November 1998, the Atrioverter was programmed in the patient-acrivated mode enabling the patient to initiate shock therapy out of the hospital by placing a magnet over the device. Over the next 6 weeks, 4 episodes of AF/arrial tachycardia were successfully cardioverted with the device. Two episodes were successfully treated by the patient out of the hospital. The other 2 episodes, initially not successfully treated with ambulatory therapy, were successfully treated with the Atrioverter when the patient came to the hospital.

\section{DISCUSSION}

Congenitally corrected transposition of the great arteries is a rare cardiac malformation, accounting for $<1 \%$ of congenital heart disease. The majority of the patients have associated cardiac abnormalities and sustained atrial arrhythmias including AF. ${ }^{3}$ Recent data on safety and efficacy of the Atrioverter in patients wirhout structural heart disease ${ }^{2}$ suggests that this device may be useful in selected patients with congenital heart disease who suffer from AF.

Because the arrhythmias of this patient persistently required external cardioversion within 24 hours and because his symptoms became more prominent over time, the remaining available treatment options were exhausted. Although interruption of AV conduction with pacemaker implantation would have resulted in ventricular rate control, it would not restore the important atrial contribution to ventricular filling during sinus rhythm. Given the favorable results of the Atrioverter in patients with $\mathrm{AF}$ and structurally normal hearts, the decision was made to implant the device in this patient. During a follow-up of 15 months, 24 spontaneous episodes of AF/arrial tachycardia occurred. Four short-lasting episodes terminated spontaneously. Of the other 20 episodes, the Atrioverter was able to restore stable sinus rhythm in 14 of them. This results in a clinical efficacy of the device of $70 \%$. Although this is lower than the $86,3 \%$ clinical efficacy reported in a recent mulricenter evaluation of the Atrioverter, ${ }^{2}$ it is acceptable given the clinical situation of the patient. Two factors seem to have been associated with the failed internal cardioversions. First, despite the effectiveness of the Atrioverter, amiodarone was reinstituted to reduce the frequency of the arrhythmia. This probably adversely affected the energy required for successful defibrillation ${ }^{4}$ and led to the decision to insert an additional defibrillation lead in the persistent left sided superior vena cava to encircle more atrial tissue, thereby lowering the defibrillation threshold. Second, one and a half months following the implanta- 
$\left.104\right|^{\text {Chapter } 9}$

tion of a third lead, the patient was admitted because of congestive heart failure and the inability to maintain a stable sinus rhythm after repeated internal and external defibrillation. Forced diuresis improved his heart failure and was followed by successful internal conversion of the same AF episode.

One of the aims of the Atrioverter is prompt defibrillation of AF outside hospital. Recently, it was shown that ambulatory therapy with the Atrioverter could terminate most episodes of AF without complications. ${ }^{5}$ Because of the absence of induction of ventricular arrhythmias during device therapy under the observation of a physician in hospital and because the patient required more than 1 hout of transportation to come to the hospital, the patient was allowed to be use the defibrillator out of hospital. Until now, 2 of 4 spontaneous episodes were successfully treated by the patient with the device outside hospital.

The implantable atrial defibrillator may be a valid trearment option in patients with congenital heart disease crippled by $\mathrm{AF}$.

\section{REFERENCES}

1. Saul JP, Walsh EP, Triedman JK. Mechanisms and therapy of complex arrhythmias in pediarric parients. J Cardiovasc Electrophysiol 1995; 6:1129-1148.

2. Wellens HJJ, Lau CP, Lüderitz B, er al. Atrioverter: An implanrable device for the treatment of atrial fibrillation. Circulation 1998; 98:1651-1656.

3. Connelly MS, Liu P., Williams WG, et al. Congenitally corrected ransposition of the great arteries in the adult: Functional status and complications. I Am Coll Cardiol 1996; 27:1238-1243.

4. Mowsowirz $\mathrm{C}$, Marchlinski FE. Interactions between implantable cardiowerter-defibrillators and class 111 agents. Am ] Cardiol 1998; 82(4A);411-481.

5. Timmermans C, Fellows C, Levy $\$$, eff an. Anbulatory use of the Metrix autonatic implantable atrial defibrillator to treat episodes of atrial fibrillation. (abstract) Eur Heart J 1998; $19: 76$. 
CHAER 10

\section{Use of sedation during cardioversion with the implantable atrial defibrillator}

Canl Tummermans, MD; Ashish Nabar, MD; Luz-Maria Rodriguez, MD;

Gregory Ayers, MD; Hein JJ Wellens, MD

From the Department of Cardiology, Academic Hospital Mastricht, Maastricht,

The Netherlands 
106 Chapter 10

\section{A B STRACT}

Background. The low shock energy used during internal atrial defibrillation may decrease the need for sedarion during defibrillation with an implantable atrial defibrillator.

Methods and Results. The atrial defibrillator (Merrix Arrioverter) was implanted in 12 patients. During the in-hospital treatment of atrial fibrillation (AF) episodes, intravenous sedarion was given only upon patient request. The Atrioverter was programmed for ambulatory therapy in 4 patients. Efficacy, number of shocks delivered and sedation requirements were recorded. A rotal of 393 shocks (1.8 \pm 1.6 shocks/AF episode) were delivered to treat 213 AF episodes; 85 of 213 AF episodes $(40 \%)$ were treated away from the hospital. Sinus thythm was restored in $195 \mathrm{AF}$ episodes $(92 \%)$. Five patients never requested sedation. No sedation was needed for ambulatory-treated AF episodes. During the treatment of 26 of 213 AF episodes $(12 \%), 75$ shocks were delivered after patients received sedation. The number of shocks required to treat an AF episode determined the need for sedation $(2.9 \pm 2.7$ shocks for AF episodes requiring sedation ws $1.7 \pm 1.3$ shocks for AF episodes requiring no sedation; $\mathrm{P}<0.001$ ). These additional shocks were needed to treat immediate reinitiation of AF (14 episodes) or initial failure to cardiovert (4 episodes). For 8 AF episodes, sedation was requested before the first shock.

Conclusions. This study suggests that, in a selected group of parients, AF can be treated with Atrioverter therapy without sedation. Successful ambulatory treatment of AF episodes with the Atrioverter, programmed to deliver $\leq 2$ shocks, did not require sedation. When mulriple shocks were required to treat an $\mathrm{AF}$ episode, the need for sedation increased and included patients inirially not requesting sedation. 
Maintaining sinus rhythm is an important goal in the management of atrial fibrillation (AF). Some of the initial skepricism regarding the value of an implantable atrial defibrillator has been answered by a recent multicenter study using the Metrix Atrioverter (InControl Inc). ${ }^{1}$ This srudy reported in a selected group of patients with recurrent $\mathrm{AF}$, accuracy of AF detection, successful cardioversion of $\mathrm{AF}$, and no ventricular proarthythmia during shock delivery. However, issues such as parient selection, therapy tolerability, effect on patient's quality of life, and cost effectiveness need ro be addressed before determining the true value of this device. The objective of this study was to evaluate the use of sedation during defibrillation with the Atrioverter as a measute of therapy tolerability.

\section{METHODS}

\section{STUDY PATIENTS}

Twelve patients with recurrent symptomatic AF and an estimated AF episode frequency ranging from I week to 3 months underwent implantation of the Metrix Atrioverter at a single center (Table 1). In the first 10 patients, the critcria used for Atrioverter implantation were as outlined in the initial Atrioverter trial. 'The device was thereafter implanted in a patient with tachycardia-induced cardiomyopathy and in a parient with a mitral valve replacement. ${ }^{2}$ Shock discomfort during pre-implant evaluation was not an exclusion criteria. After device implantation, the parients continued to receive antiarrhythmic drugs as needed. At the completion of this study in October 1998, the mean follow-up duration was $17.3 \pm 6.5$ months (range, 5 to 25 months?.

\section{ATRIOVERTER THERAPY}

Only spontaneous AF episodes for which cardioversion wirh the Atrioverter was attempred were analyzed. Biphasic shocks with a programmable voltage $(200,240$, 260 , or $300 \mathrm{~V})$ were delivered to treat AF episodes. Shocks were delivered benween coil electrodes, 1 in the right atrium and 1 in the coronary sinus. During at least the first 3 months after implantation, the device was programmed in monitoring mode, and all shocks were delivered in the presence of a physician. Patients were forewarned when the device was acrivated to deliver therapy. Sedation was nor used on a routine basis, but it was provided on patient request; intravenous midazolam, etomidate, or diazepam wiss used. In cases of failure to restore sinus rhythm or when immediate reinitiation of AF occurred (IRAF), ${ }^{3}$ intravenous flecainide (50 to 150 $\mathrm{mg}$ ) or sotalol (40 to $120 \mathrm{mg}$ ) was given before the next shock.

The Atrioverter was programmed for out-of-hospital therapy in 4 patients. These 4 parients had frequent episodes of $\mathrm{AF}(2.7 \pm 1.8 \mathrm{AF}$ episodes/month) that were 
Table 1. - Bastine characteristics of the 12 patients studied.

\begin{tabular}{|c|c|}
\hline Age, y & $57 \pm 9$ \\
\hline Menfwomen & $9 / 3$ \\
\hline Duration of $A F$ as disease, $y$ & $11.8=7.6$ \\
\hline Failed AAD preimplant, median (range) & $4(3-6)$ \\
\hline \multicolumn{2}{|l|}{ Structural hear discalise, n } \\
\hline $\mathrm{CAD}$ & 1 \\
\hline Tactycardiomyoparthy & 1 \\
\hline Hypertension & 3 \\
\hline MVR & 1 \\
\hline None & 6 \\
\hline Left atrial size, cm & $5.1 \pm 0.5$ \\
\hline Metrix Atrioverrer model $3000 / 3020$ & $1 / 11$ \\
\hline \multicolumn{2}{|l|}{ Postimplantarion AAD, n } \\
\hline Class Ic & 5 \\
\hline Class IIII & 4 \\
\hline Combination dass Ic and IIII & 3 \\
\hline Follow-up duration, mo (range) & $17.3 \pm 6.5(5-25)$ \\
\hline
\end{tabular}

AAD: Antiarthythruic drugs; CAD: Coronary attery disease; MVR: Mirral valve replacement.

treated initially in-hospital with the Atrioverter. Ambulatory use of the device was considered for the convenience of the patient. The device was programmed to deliver 1 shock of $300 \mathrm{~V}$ in 3 parients and 2 shocks of $300 \mathrm{~V}$ in the remaining patient. Parients were instructed to come to the hospital for further treatment of an AF episode if the programmed shock(s) failed to restore sinus rhythm.

\section{STATISTICAL ANALYSIS}

Results are expressed as mean $\pm \mathrm{SD}$. Continuous variables were compared using Student's r-test or ANOVA for unpaired samples. All results were considered significant at $P<0.05$.

\section{RESULTS}

\section{ATRIOVERTER THERAPY}

During a mean follow-up of $17.3 \pm 6.5$ months, 12 patients were treated for 213 spontaneous episodes of AF for which 393 shocks were delivered (mean $1.8 \pm 1.6$ shocks/AF episode; median, 1 shock/AF episode) by the Atrioverter (Table 2). No 
Table 2. - Results of Atrioverter therapy.

\begin{tabular}{|c|c|}
\hline Mean number of AF episodes/patien (range) & $17.6(2-70)$ \\
\hline Median duration of treated AF episode, h (range) & $17(1-164)$ \\
\hline Mean number of shocks/AF episode (range) & $1.8 \pm 1.6(1-13)$ \\
\hline \multicolumn{2}{|l|}{ Device activrated, No. of AF episodes } \\
\hline By physician & $129(60 \%)$ \\
\hline By parient & $85(40 \%)$ \\
\hline \multicolumn{2}{|l|}{ Shock strength used, No of shocks } \\
\hline $300 \mathrm{~V}$ & $360(91.6 \%)$ \\
\hline $260 \mathrm{~V} / 240 \mathrm{~V} / 200 \mathrm{~V}$ & $14 / 18 / 1$ \\
\hline \multicolumn{2}{|l|}{ Outcome of device-treated AF episodes } \\
\hline Restored sinus thythm & $195(91.50 \%)$ \\
\hline Restored sinus rhythm with $\mathrm{AADs}_{s}^{*}$ & $7(3.3 \%)$ \\
\hline Unable to restore sinus rhythm & $11(5.2 \%)$ \\
\hline Occurrence of IRAF, No. of AF cpisodes & $45(21.3 \%)$ \\
\hline
\end{tabular}

AAD Antiarrhythmic drug. ${ }^{*}$ Sinus rhythm was restored after in travenous AAD administered before the nexr shock.

differences existed between the patients with respect to the mean number of delivered shocks per AF episode ( $\mathrm{p}=0.25)$. Overall, the device successfully terminated 195 AF episodes ( $92 \%)$, usually using a single $300-V$ shock. During ambulatory therapy, the Atrioverter restored sinus rhythm for 66 of the $85 \mathrm{AF}$ episodes $(78 \%)$. For the remaining 19 episodes, subsequent device therapy successfully restored sinus rhythm when the patients came to the hospital. During the treatment of 45 of 213 AF episodes ( $21 \%)$, IRAF was observed.

\section{SEDATION REQUIREMENTS}

Five of the 12 patients did not request sedation during the treatment of any AF episode. Sedation was requested by the remaining 7 patients during the treatment of 26 of 213 of the AF episodes (12\%; 75 of 393 shocks, 19\%). No sedation was required during the treatment of $187 \mathrm{AF}$ episodes (88\%). Furthermore, for the 85 episodes treated while the parients were away from the hospital, no sedation was required.

Conscious sedation was provided using intravenous midazolam (19 AF episodes), etomidate (5 AF episodes), or diazepam ( $2 \mathrm{AF}$ episodes), with a mean dose of $5.7 \pm 6$ (range, 2 to 30 ), $22 \pm 8$ (range, 8 to 40 ), and $10 \pm 7$ (range, 5 to 15 ) $\mathrm{mg}$, respectively. In general, sedation was needed only when a greater number of shocks was needed to treat an AF episode (2.9 \pm 2.7 shocks for AF episodes requiring sedation versus $1.7 \pm$ 1.3 shocks for $\mathrm{AF}$ episodes requiring no sedation; $\mathrm{p}<0.001)$. As illustrated in the Figure, the percentage of $A F$ episodes requiring sedation increased as the number of 


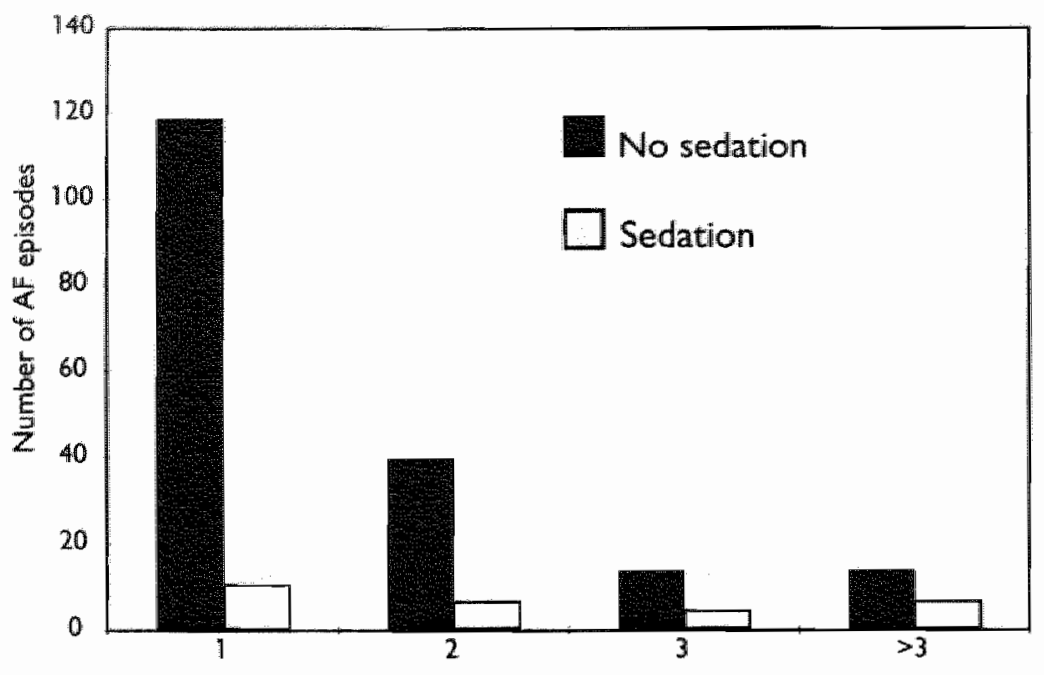

Number of shocks for successful treatment of an AF episode

Figure - The need for intravenous sedation during trearment of spontaneous AF ephodes using the Atriowerter.

shocks delivered per AF episode increased. Sedation was requested during the treatment of $30 \%$ of AF episodes requiring $>3$ shocks.

The most frequent cause for sedation was the delivery of multiple shocks required to trear IRAF (14 of 26 episodes). Other reasons for the use of sedation included a request for sedation before the deliwery of the first therapeutic shock ( 8 of 26 episodes) and initial failure to cardiovert ( 4 of 26 episodes).

\section{DISCUSSION}

Internal atrial defibrillation was initially considered a method that would diminish the need for sedation by decreasing the shock intensity required for successful cardioversion. However, in an early study of this technique in which defibrillation thresholds were determined using a step-up defibrillation protocoll, the majority of the patients required sedation. ${ }^{4}$ Conversely, other studies have shown that low energy internal atrial defibrillation can be performed in most cases without sedat tion. 5.6 Lok et al., suggested that the number of shocks substantially affecrs discomfort and that $2-3$ shocks are usually rolerated. ${ }^{5}$ Therefore, it is likely that the number of shocks, as much as their individual intensiry, affect patient discomfort. In the present study, Arrioverter therapy could be delivered without sedation when 1.7 \pm 1.3 shocks/AF episode were delivered, but sedation was used when $2.9 \pm 2.7$ shocks were required per AF episode. As shown in the Figure, the request for sedation increased with the number of shocks delivered to treat an AF episode. Further- 
more, sedation was required at 1 rime or another by 7 of the 12 parients but only during the treatment of 26 of 213 AF episodes (12\%). These findings further indicate a relationship between the number of shocks delivered and the need for sedation.

Controlling the reasons for patients to request sedation during Arrioverter therapy will improve the acceptance of the device. Because IRAF was the most frequent cause for the delivery of additional shocks and, consequently, for the use of sedation during Atrioverter therapy, successful management of IRAF could further decrease the need for sedation and improve overall acceptance of the therapy. Suppression or prevention of premature atrial complexes that initiate AF by pharmacological therapy ${ }^{3}$, ablation ${ }^{7}$ or pacing may preserve sinus rhythm after successful cardioversion without the need for additional shocks. Also, requests for sedation before delivery of the first shock could be decreased by psychologically preparing the parient, early initiation of ambulatory therapy to encourage self-confidence and, when necessary, through the use of anxiolytic drugs. Further improvements in device technology ${ }^{8}$ and a better understanding of the influence of concomitant therapy may contribute to lowering the defibrillation threshold. Lowering the defibrillation threshold could increase the rate of successful cardioversion at lower energies, which in rurn, may reduce the need for sedation during Atrioverter therapy.

\section{STUDY LIMITATION}

Overall patient acceptance of this therapy and individual shock tolerability, as determined by using a questionnaire, were not evaluated during this study. What was measured was the need, on the basis of parient request, for agents intended to relieve the discomfort of the therapy. However, it is important to note that parients continued to desire to receive Atrioverter therapy without sedation, even when sedation may have been required for a prior episode. This finding is indicative of, but not conclusive for, overall Atrioverter patient tollerability.

\section{CONCLUSIONS}

This study suggests that, in a selected group of patients, AF episodes can be successfully treated with the Arrioverter without the use of sedation. Successful ambulatory reatment of $A F$ episodes with the Atrioverter, programmed to deliver $\leq 2$ shocks, did not require sedation. When multiple shocks are required for the trearment of an AF episode, the need for sedation increases. Reduction in threshold may reduce the discomfort caused by individual shocks; however, furure efforts should be focused on reducing the number of shocks. Additionally, furure studies are needed to evaluate the patients' perceived quality of life and overall patient acceptance of therapy. 


\section{REFEREHCES}

1. Wallens HJ], Law CP, Luderitz B, Akhod M, Waldo A, Camm Af, Timmermans C, Tse HF, Jung W, Jordaens $\mathrm{L}$. Ayers $\mathrm{G}$ for the Merrix Investigators. Arrioverier: an implantable device for the treatment of atrial Gbrillarion. Circulation. 1998;98:1651-1656.

2. Timmermans C, Rodriguez LM, Ayers GM, Siu A, Smeets J, Barenbrug P, Wellens HJ for the Metrix Expanded Indication Investigators. Design and preliminary data of the Metrix Arrioverter expanded indication trial. J Interv Card Electrophysiol. In press.

3. Timmermans $\mathrm{C}$, Rodrigucz LM, Smeets JLRM, Wellens HJJ. Immediate reinitiation of atrial fibrillation following internal atrial defibrillation. I Cardiovasc Electrophysiol. 1998;9:122-128.

4. Murgatroyd FD, Stade AKB, Sopher M, Rowland E, Ward DE, Camm AJ. Efficacy and tolerability of transvenous low energy cardioversion of paroxysmal atrial fibrillation in humans. I Am Coll Cardiol. 1995;25:1347-1353.

5. Lok NS, Lau CP, Tse HF, Ayers GM. Clinical shock tolerability and effect of different right atrial electrode locations on efficacy of low energy human transvenous atrial defibrillation using an implantable lead sysrem. J Am Coll Cardiol, 1997;30:1324-1330.

6. Timmermans C, Rodriguea LM, Ayers GM, Lamberr H. Smeets ILRM, Vlaeyen JWS, Albert A, Wellens HJJ. The effect of butorphanol tartrate on shock related discomfort during internal arrial defibrillation. Circulation. 1999;99:1837-1842.

7. Lau CP, Tse HF, Ayers GM. Defibrillation-guided radiofrequency ablation of atrial fibrillation secondary to an atrial focus. I Am Coll Cardiol. 1999;33:1217-1226.

8. Cooper RAS, Plumb VJ, Epstein AE, Kay GN, Ideker RE. Marked reduction in internal atrial defibrillation thresholds with dual-current pathways and sequential shocks in humans. Citculation. 1998:97:2527-2535. 


\section{Initial clinical experience with ambulatory use of an implantable atrial defibrillator for conversion of atrial fibrillation}

Emile G Daoudt, MD; Carl Timmermans* , MD; Chris Fellowst, MD;

Robert Hoy\$, MD; Robert Lemery 4 , MD; Kathy Dawson ${ }^{* *}, \mathrm{PhD}$

Gregory M Ayers**, MD, PhD; for the Metrix Investigatorsti

$†$ Riverside-Methodist Hospital, Columbus, OH, USA: *Acadenic Hospital Maastricht, Maastricht, the Nerherlands; NVirginia Mason Medical Center, Seattle, WA, USA; \$lowa Hearr Institute, Des Moines, IA, USA; 9Rhode Istand Hospital Prowidence, RI, USA; and * InControl, Ine, Redmond, WA, USA tT The Merrix Investigators are listed in the Appendix.

Abstract presented at the 20 h Ammal Scientifu Sessions of the Now American Society of Pacing and Electrophysiology 1998 (Pacing Ciw Electrophysiol 1998;21:81)

(Submitted for publication) 


\section{A B STRACT}

Objective. The objecrive of this study was to evaluate the safery and efficacy of ambulatory use of the Metrix implantable atrial defibrillator and determine the impact of this therapy on the patient.

Background. A recent study has shown that the implantable atrial defibrillator could repearedly restore sinus rhythm in patients with recurrent atrial fibrillation when therapy was delivered under physician observation. However, little is known about the use of this device when the patient is ambulatory and receiving therapy while not under direct physician supervision.

Methods. An atrial defibrillator was implanted in 105 patients $(75$ men, mean age 59 \pm 12 years) with recurrent, sympromatic, drug-refractory atrial fibrillation. For at least the first 3 months after implant, the device was programmed to a monitoring mode with episodes of atrial fibrillation defibrillated under physician obserwation. After a successful 3-month follow-up testing, physicians were allowed to program the device for either patient-activated or automatic therapy for subsequent episodes of atrial fibrillation. Patients were asked to complete questionnaires that assess severity of symptoms, therapy discomfort and satisfaction with therapy using a 10-point visual-analog scale after each treated atrial fibrillation episode.

Results. During a mean follow-up of 11.7 months, $75 / 105$ patients had experienced one or more episodes of atrial fibrillation. These patients received device therapy for 663 episodes of which 388 were treated prior to transition to ambulatory therapy. Of the 105 patients, 48 had reached ar least 3 months follow-up and met the transition criteria. Patients were allowed to self-activate their device $(\mathrm{N}=42)$ or had their device programmed to automatic therapy delivery $(\mathrm{N}=6)$. The 48 patients received therapy for 275 episodes with a therapy efficacy of $90 \%$ (mean $87 \%$, median $100 \%$ ) for which an average of $1.6 \pm 1.2$ shocks per episode were delivered (median 1). For successful therapy, patients rated the discomfort as $5.2 \pm 2.4$ and $4.2 \pm 2.2$ for unsuccessful therapy $(p>0.05)$. The satisfaction score was lower for unsuccessful as compared to successful therapy $(3.4 \pm 3.3$ vs. $8.7 \pm 1.3, \mathrm{p}<0.05)$. There was no ventricular proarrhythmia observed associated with the delivery of the 5523 shocks. Conclusions. Ambulatory use of the implantable atrial defibrillator has shown that most episodes of atrial fibrillation can be safely and successfully treated. Successful atrial defibrillacor treatment of atrial fibrillation is associated with high therapy satisfaction and only moderate shock discomfort. 
Electrical cardioversion is an established technique for restoration of sinus rhythm in patients with atrial fibrillation ${ }^{1}$. Defibrillation using transvenous catheter-based electrodes has been shown to be safe and effective for restoration of sinus rhythm, even in patients who have failed artempts to restore sinus thythm with transthoracic shocks ${ }^{2-4}$. When proper R-wave synchronized shocks are delivered to a carheter-based system, conversion of atrial fibrillation can be achieved with low amounts of energy (1-6 J). From this experience, an implanted atrial defibrillaror was developed to treat patients with recurrent, symptomatic, drug-refractory atrial fibrillation. An implantable atrial defibrillator was initially evaluated in a prospective, multicenter study. In this study, the device accurately detected and safely cardioverted symptomatic episodes of atrial fibrillation, however, device acrivation and therapy delivery was performed in the hospital 5,6 . The purpose of this study was to evaluate the ambulatory use of this atrial defibrillator. In particular, this study assessed the ability of the implantable atrial defibrillator to accurately detect and safely cardiovert episodes of atrial fibrillation and evaluated the therapy tolerability in the outpatient setting.

\section{METHODS}

\section{PATIENT POPULATION}

The atrial defibrillator was implanted in 105 patients with recurrent, symptomatic, d.rug-refractory atrial fibrillation. All patients provided written informed consent and the protocol was approved by the Hospital Institutional Review Board or Erhics Committee. Inclusion and exclusion criteria were previously described ${ }^{5}$. Concomitant pharnacologic trearment was documented, however, the choice of and changes in drug therapy was left to the preference of the physician.

\section{ATRIAL DEFIBRILLATOR}

An atrial defibrillator (Metrix model 3000 and 3020; InControl, Inc., Redmond, WA), was implanted in conjunction with two defibrillation leads and a bipolar pacemaker lead. The leads were introduced through the subclavian vein and the defibrillation leads were positioned in the right atrium (model 7205) and distal coronary sinus (model 7109). The pacemaker lead was placed in the right ventricle (Figure 1). The earlier atrial defibrillator generator (model 3000) had a maximum output of $3 \mathrm{~J}$. The next generation device (model 3020 ) had a maximum output of $6 \mathrm{~J}$. Each model delivered a biphasic waveform shock. The defibrillator can be programmed to provide up to 8 shocks, with individual voltages programmable from 20 to $300 \mathrm{~V}$ in $10 \mathrm{~V}$ increments. The device uses a dual-algorithm to detect atrial 

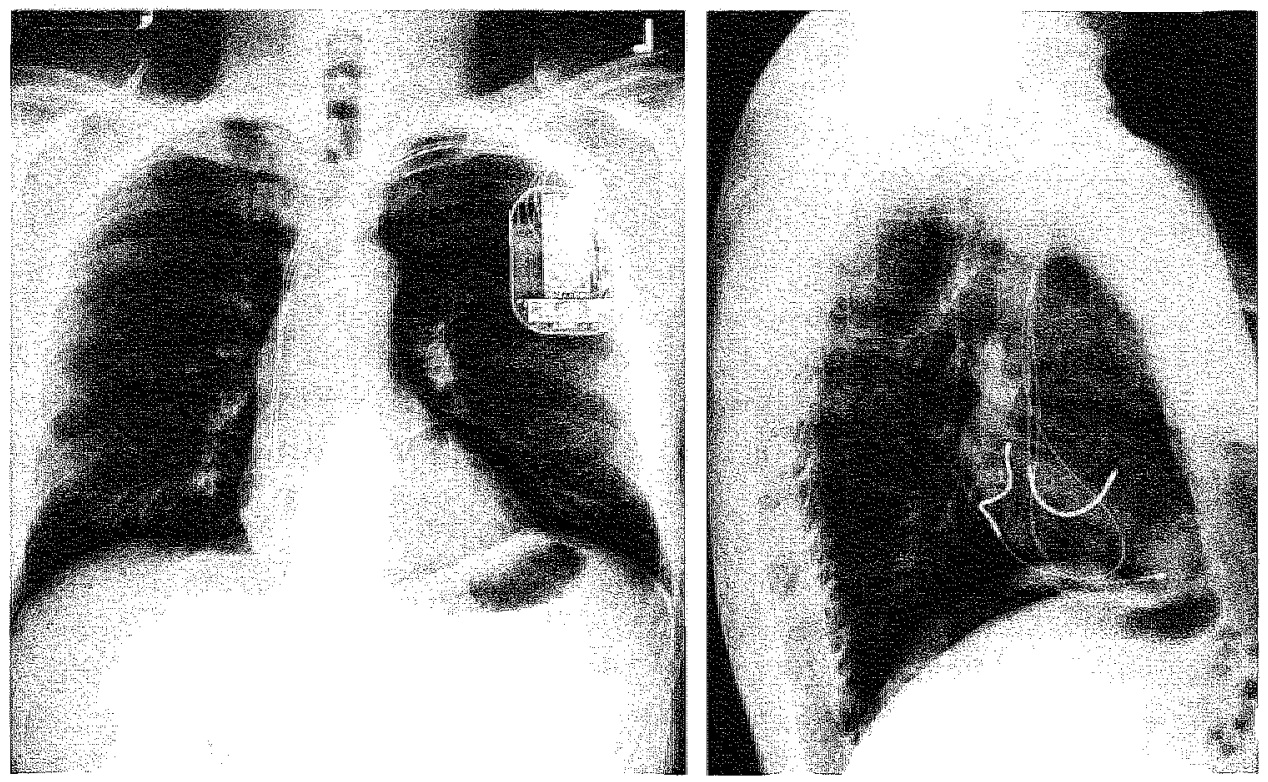

Figure 1.- Anteroposterior (left panel) and lateral (right panel) chest $x$-ray of a patient implanted with a Metrix arrial diefibrillator. Seen in the $x$-ray are the three leads, one defibrillation lead in the distal coronary sinus, a second defibrillation lead in the right atrum and a bipolar lead in the right ventricle. The pulse generator is in the lefic pectoral region.

fibrillation and defibrillation shocks are synchronized to the R-wave ${ }^{6}$. The device also manages postshock bradycardia with VVI pacing.

The atrial defibrillator can be programmed to one of 5 operating modalities: automatic, patient-activated, monitor, pacing-only and off mode. In the automatic mode, the device is programmed, from every minute to 120 minutes, to monitor the cardiac rhythm for the presence of atrial fibrillation. Therapy will be dellivered if arrial fibrillation is present, unless the patient inhibits device therapy with a conventional pacemaker magner. The parient-activated mode allows the parient to activate the device with a pacemaker magnet. If the device detects atrial fibrillation, therapy will be delivered unless inhibired by a magner. The monitor mode functions like the automatic mode with respect to atrial fibrillation detection, however, the device does not deliver defibrillation therapy. For the modes where therapy is delivered, there is the option to program a warning shock $(10-20 \mathrm{~V})$ preceding therapy delivery. When programmed to the pacing-only mode, the device acts as a VVI pacemaker.

The device stores 3 electrograms for each episode. The atrial fibrillarion detection electrogram displays the atrial and ventricular signals. The second electrogram captured by the device is from the period just prior to the delivery of shock therapy, displaying the ventricular electrogram with a mark on the $\mathrm{R}$-wave where the shock was delivered. The third electrogram is the same as the first, except that the electrogram is from the period after the shock. The investigators used these 
electrograms to assess the accuracy of device based atrial fibrillation detection, to confirm synchronization, to evaluate the outcome of shock therapy, and to detect ventricular proarrhythmia.

\section{POSTIMPLANTATION FOLLOW-UP}

For at least the first 3 months after implant, the atrial defibrillator was programmed to the monitor mode. Patients were instructed to return to the hospital or clinic for treatment of each symptomatic episode of atrial fibrillation. Defibrillation was then performed under physician observation during ECG recording. After at least 3 months of follow-up, the following criteria had to be met before patients could use their device outside the hospital/clinic: (1) an atrial defibrillarion energy requirement $\leq 260 \mathrm{~V}$; (2) no false positive detections of atrial fibrillation; (3) correct R-wave synchronization; (4) patient education regarding device activation/deactivation; and (5) at least one episode of atrial fibrillation treated under physician observation that met each of the following requirements: a) successful termination with $\leq 300 \mathrm{~V}$; b) therapy was delivered under the same conditions as planned for ambulatory use; $c$ ) the patient tolerated the therapy; and d) sinus rhythm was maintained for one week after successful termination. In addition to fulfilling these criteria, the patient needed to agree with being rreated using ambulatory therapy. Thereafter, the device was programmed to the patient-activated or the automatic mode. The decision about the mode of device programming (automatic vs. patient-activated) was left to the discretion of the physician in conference with the patient.

The patient was instructed to complete an event form and to return for clinical follow-up after each treated episode of atrial fibrillation. The event form recorded the patient-perceived onset of symptoms and assessed their perception of atrial fibrillation and device therapy. A visual-analogue scale (1 represented "not at all", 10 represented "extremely") was used to score the answers to the following questions: (1) "How severe were the symptoms from this episode of atrial fibrillation?"; (2) "How satisfied were you with the therapy?"; (3) "How uncomfortable was the therapy?". The fourth question required a yes/no response to, "Did device therapy relieve symptoms?".

Using the stored electrograms and the patient's report of symptoms, the investigator determined whether an individual episode of atrial fibrillation was terminated. Ambulatory efficacy was defined as the percentage of episodes terminated by the device when treated away from the hospital/clinic. Therapy efficacy was defined at the overall percentage of episodes converted by the atrial defibrillator to stable sinus rhythm, either with treatment delivered while the patient was ambulatory or when the patient was treated in the hospital/clinic. 
Data were collecred from the patient and the hospital record about the frequency of in patient hospital and emergency room admissions for the 6 months prior to implant, not including the admission for implant of the device.

\section{STATISTICAL ANALYSIS}

Conrinuous variables are expressed as mean and standard deviation and were compared using an analysis of variance. Population safety and efficacy calcularions were made by fitting the distributions of these variables in an individual patient (assuming a exponential distribution for risk of proarrhythmia and a binomial probability distribution for efficacy) then determining the mean across the population of patients, assuming a beta distribution. Similarly, the risk of proarrhythmia was calculated for the patient population. For all analyses, a p-value $<0.05$ conferred staristical significance.

\section{RESULTS}

\section{PATIENT CHARACTERISTICS}

Parient characteristics are summarized in Table 1 and antiarrhythmic therapy at time of last follow-up is summarized in Table 2. Structural heart disease was present in 78 patients $(74 \%)$ and patients failed $4.8 \pm 2.1$ antiarrhythmic drugs. Antiplatelet therapy or anticoagulation was prescribed at the discretion of the investigator. There were no thromboembolic events.

Of the 105 patients enrolled, the device was explanted in 15 parients. Three devices (3\%) were explanted because of lead complications, one due to infection $(1 \%)$ and $11(10 \%)$ were explanted because of inadequate control of atrial fibrillation. In the 105 patients, 18 of the $270(7 \%)$ leads implanted required repositioning. The coronary sinus lead did not dislodge in any patients. The mean follow-up was $11.7 \pm 6.8$ months and 87 parients had a follow-up of more than three months.

TREATMENT OF ATRIAL FIBRILLATION: PRIOR TO TRANSITION TO AMBULATORY THERAPY

Seventy-five patients had 412 episodes of atrial fibrillation for which they sought therapy. Of these 412 episodes, 388 were treated with 897 shocks delivered from the atrial defibrillator. The atrial defibrillator restored sinus rhythm in 368 of the 388 episodes (95\%), however atrial fibrillation recurred within minutes after successful cardioversion for 95 episodes (early recurrence of atrial fibrillarion) and prevented 
Table 1. - Demographics of the 105 srudied patients.

\begin{tabular}{ll} 
Age & $59 \pm 12(25-79)$ yrs. \\
Gender & $75(72 \%)$ \\
Men & $30(28 \%)$ \\
Women & $57 \pm 8(38-78) \%$ \\
LVEF & $4.5 \pm 2,1(3.3-8.0) \mathrm{cm}$ \\
LA size & 99 \\
NYHA dass (SR) & $83(84 \%)$ \\
I & $16(16 \%)$ \\
II & 81 \\
NYHA class (AF) & $61(75 \%)$ \\
I & $11(14 \%)$ \\
II & $8(10 \%)$ \\
III & $1(1 \%)$ \\
IV & \\
Structural Hear Disease & 27 \\
None & 46 \\
Hypertension & 22 \\
Coronary Artery Disease & 8 \\
Prewious MI & 12 \\
PTCA & 9 \\
CABG & 36 \\
Valwular Heart Disease & 2 \\
Repair/Replacement & 2 \\
Congenital Heart Disease & \\
Cardiomyopathy & \\
\hline
\end{tabular}

AF: atrial fibillation; $C A B G$ : coronary artery bypass grafring; LA: left atrium; LVEF : left ventricular ejection fraction: MI: myocardial infarction; PTCA: percutaneous transluminal coronary angioplasty; SR: sinus thythm. Dara are presented as tnean standare deviation and nanger of number of pacierts and when appropriare, percentage.

the restoration of stable sinus rhythm. For 54 of the 95 episodes where early recurrence of atrial fibrillation occurred during the treatment of an episode, sinus rhythm was eventually restored using additional shocks from the atrial defibrillator with or without the administration of antiarrhythmic drugs. Taking into account the remaining 41 of the 95 episodes where early recurrences were observed and could not be successfully treated the therapy efficacy was $85 \%$.

\section{TREATMENT OF ATRIAL FIBRILLATION: AMBULATORY THERAPY}

At the time of the most recent follow-up, 48 patients fulfilled ali criteria and agreed to receive ambulatory therapy. The reasons why a specific patient did not transition 
Tabte 2. - Anvarty hhmic drug use ar time of last clinical follow-up.

\begin{tabular}{lc}
\hline & Last follow-up \\
\hline None & $9(9 \%)$ \\
Rate control only & $14(13 \%)$ \\
Class I & $23(22 \%)$ \\
Class II & \\
$\quad$ Sotalol & $24(23 \%)$ \\
$\quad$ Arniodarone & $35(33 \%)$
\end{tabular}

Data are presented as the number of parients and percentage.

to ambulatory therapy were not documented. For six patients, the device was programmed to the automatic mode and for 42 patients the device was programmed to patient-activated mode. Table 3 outlines the programmed number of shocks and their intensity from 43 of 48 patients in whom these data were avallable at the time of most recent follow-up. The mean number of shocks programmed was $2.1 \pm 1.0$, with a median of 2 . No patients had more than four shocks programmed. The first shock voltage was programmed to the maximum output of $300 \mathrm{~V}$ for 28 of the 43 patients (65\%). Second, third and fourth shocks were programmed to maximum ourput. A warning shock was programmed on for 18 patients (42\%). In most patients, this low voltage shock was delivered within the 5 minutes prior to the delivery of the therapeutic shock.

Of the 48 patients, 37 patients had 301 episodes of atrial fibrillation for which they desired therapy. Accurate and complete data to assess therapy outcome was available for $296 / 301$ episodes $(98 \%)$. The mean follow-up after transition to ambulatory therapy was $8.4 \pm 3.7$ months. The average number of episodes per patient was $8 \pm 10$ (range, 1 to 43 ) with a median of 4 episodes/patient. The adjusted monthly episode rate for this patient population was $1.4 \pm 1.0$ episode/patient-month (range, 0.1 to 4.6) with a median of 1.2 episodes/parient-month.

A rocal of 365 shocks were delivered for ambularory therapy, all for atrial fibrillarion confirmed using the stored electrograms. No shocks were delivered when the patients were in sinus rhythm. The mean number of shocks required to treat each episode of atrial fibrillarion was $1.6 \pm 1.2$ (range, 1 to 8 ) with a median of 1 shock/episode. In 276 of the 296 episodes of atrial fibrillation where the number of shocks delivered was known, 191 (69\%) could be successfully treated with 1 shock. and only $8 \%$ of episodes required more than 3 shocks.

The ourcome of the 296 episodes is summarized in Figure 2. For 265/296 episodes of atrial fibrillation (90\%), the patient attempted to treat the episode. The atrial defibrillator successfully converted $199 / 265$ episodes (75\%) and therapy failed to successfully converr 52 episodes (25\%). The remaining 14 episodes did not result in defibrillator therapy delivery because either normal device function precluded delivery of therapy due to a thigh ventricular rate ${ }^{6,7}$, the patient did not correctly acti- 
Table 3n - Atrial defibrilator programmed parameters for ambularory therapy from 43 parients with dic Merrix implantable arrial defibrillanor.

\begin{tabular}{llc}
\hline & & Nurnter of parients \\
\hline Number of shocks programmed & 1 & $12(28 \%)$ \\
& 2 & $20(47 \%)$ \\
& 3 & $6(14 \%)$ \\
1st Shock Volrage & 4 & $5(12 \%)$ \\
& $220 \mathrm{~V}$ & $2(5 \%)$ \\
& $240 \mathrm{~V}$ & $1(2 \%)$ \\
& $260 \mathrm{~V}$ & $12(28 \%)$ \\
2nd Shock Voltage & $300 \mathrm{~V}$ & $28(65 \%)$ \\
3 rd Shock Voltage & $300 \mathrm{~V}$ & $32(100 \%)$ \\
4th Shock Voltage & $300 \mathrm{~V}$ & $11(100 \%)$ \\
Warning Shock & $300 \mathrm{~V}$ & $5(100 \%)$ \\
& Yes & $18(42 \%)$ \\
& No & $25(58 \%)$ \\
\hline
\end{tabular}

vate the device with the magnet, or the patient deactivared the device after initial activation. Of the 66 episodes of atrial fibrillation that were not successfully treated or did not receive therapy, 34 converted spontaneously $(51 \%)$ after failed device therapy $(n=22 / 52)$, or after ambulatory was nor delivered $(n=12 / 14)$.

For the remaining $31 / 296$ episodes of atrial fibrillation, patients chose to return to the clinic/hospital for therapy for 27 episodes and, atrial fibrillation converted spontaneously for 4 episodes. Atrial fibrillation also converted spontaneously in 4 of the 27 episodes for which the patient chose to return to the clinic/hospital for device therapy.

The 55 sustained episodes of atrial fibrillation were treated under physician observation (lower half of Figure 2). Of these 55 episodes, $49(89 \%)$ were successfully treated by the physician with the atrial defibrillator, 5 episodes failed to be terminated and later reverted spontaneously to sinus rhythm, and 1 episode terminated prior to physician activated therapy in the clinic. When considering episodes of atrial fibrillation that were converted with the defibrillator, the therapy efficacy of the defibrillator was $248 / 275(90 \%)$. The individual patient therapy efficacy was 87 $\pm 27 \%$ with a median of $100 \%$. Therapy efficacy was $100 \%$ in $70 \%$ of patients, and $3 / 37$ patients $(8 \%)$ had only a single episode of atrial fibrillation in which the arrial defibrillator therapy failed to cardiovert the episode. 
122 Chapter 11

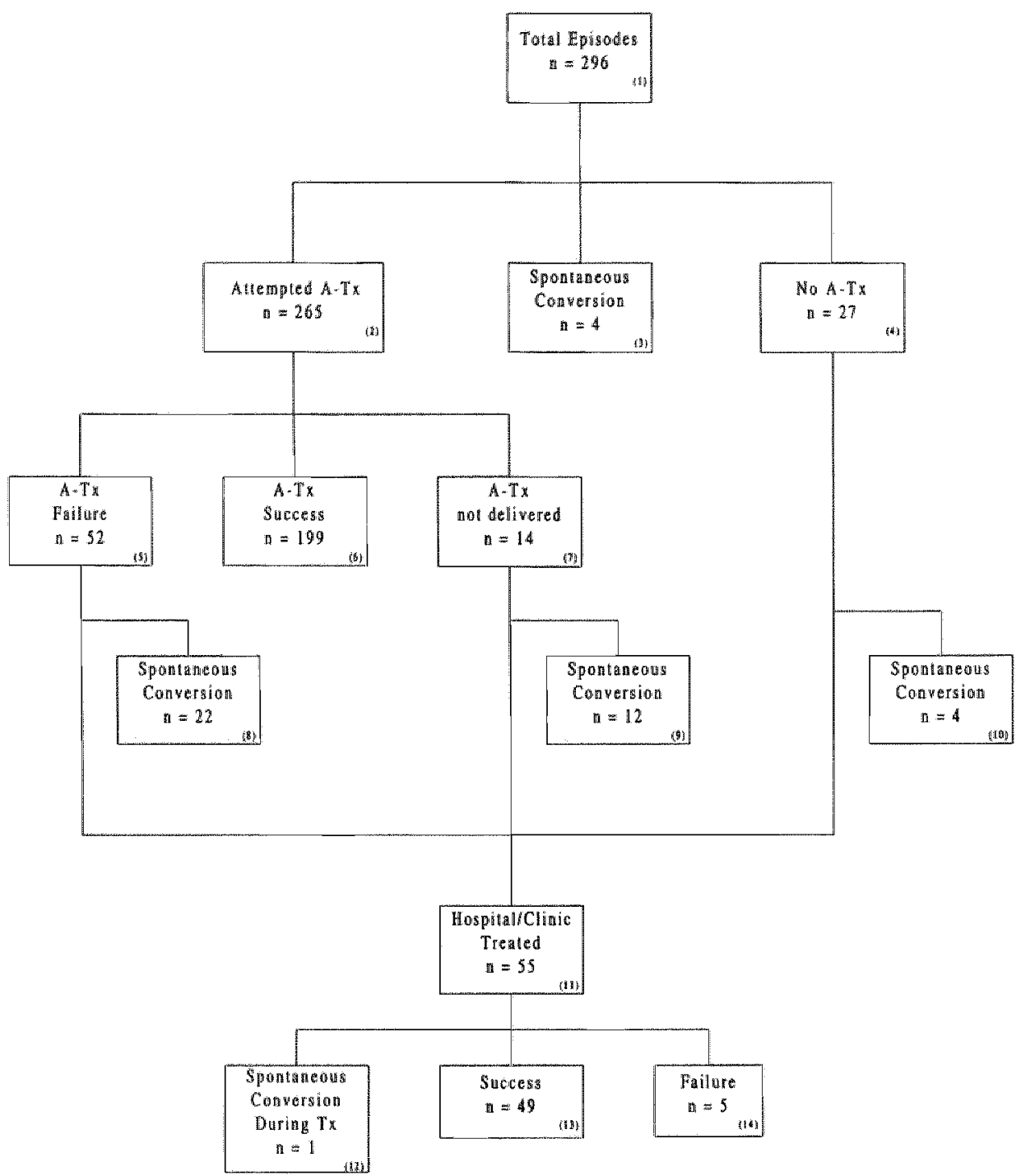

Figure 2. - Flow-chat showing the outcome of ambulatory treated arrial fibrillacion episodes with the atrial defibrillaror. (Note: the numbers within parentheses in the right upper corner of each box are used in the equavions to describe the method for the deternimation of the therapy efficacy of the ambulatory use of the device). A: ambularory: n: number of atrial fibrillation cpisodes; Tx: therapy. 


\section{SAEETY OF SHOCK THERAPY}

During this study, there were 5523 shocks delivered from the arrial defibrillator, of which 365 shocks were delivered during ambulatory therapy. An additional 897 shocks were delivered to rreat episodes of atrial fibrillation prior to the parient's transition to ambulatory therapy. The remaining 4261 shocks were delivered for atrial fibrillation induccion and defibrillation testing. All shocks were delivered with appropriate synchronizarion and there were no episodes of ventricular proarrhythmia observed. Taking into account the number of shocks/patient and the number of patients enrolled in this study, the estimated mean proarhythmic risk was found to be $0 \%$ with the 95th percentile upper confidence interval of risk of proarrhythmia of $0.056 \% /$ shock. Based on the data from this study (the median monthly episode rate and shocks per episode), the maximum annual risk of proarrhythmia would be $0.81 \%$.

\section{PERCEPTION OF AMBULATORY THERAPY}

The impact of event scores were available from 143 treated episodes ( 25 patients). The mean number of episodes per patient was $5.7 \pm 6.1$ (range, 1 to 32) with a median of 4 episodes/patient.

The average symptom severity score was not significantly different when comparing episodes that were successfully treated as compared with those unsuccessfully treated $(6.3 \pm 2.0$ vs. $5.2 \pm 1.8, p>0.05)$. The overall severity index was $6.1 \pm$ 1.9. Similarly, there was no significant difference between successfully and unsuccessfully treated episodes with respect to discomfort $(5.2 \pm 2.5$ (median, 5.0) vs. 4.2 \pm 2.2 (median, 4.0), p >0.05). Patients did have significantly higher sarisfaction scores associared with successful as compared to unsuccessful therapies $(8.7 \pm 1.3$ (median, 9) vs. $3.4 \pm 3.3$ (median, 2), p<0.05). Patients reported relief of symptoms after trearment of $83 \%$ of episodes.

\section{DETERMINATION OF HEALTH CARE UTILIZATION}

The percentage of patients with emergency room visits and in-patient hospital admissions for atrial fibrillation are shown in Table 4. As can be seen, the patients enrolled in this study had substantial contact with the health care system, with most parients having either an emergency room visit or inparient admission prior to implant of the device. 
Table 4. - Percentage of patients with emergency roon visits and in-patient hospitalizations in the 6 monchs prior to implant.

\begin{tabular}{lc}
\hline Energenoy Rowom Visits & Prior to Implant $N=63$ \\
0 & $44 \%$ \\
1 & $19 \%$ \\
2 & $10 \%$ \\
3 & $16 \%$ \\
4 & $5 \%$ \\
5 & $6 \%$ \\
Hospital Admissions & \\
0 & $24 \%$ \\
1 & $22 \%$ \\
2 & $18 \%$ \\
3 & $16 \%$ \\
4 & $8 \%$ \\
5 & $13 \%$ \\
\hline
\end{tabular}

\section{DISCUSSION}

\section{MAIN FINDINGS}

The main findings of this study are that an implanted atrial defibrillator can be utilized for outpatient electrical cardioversion of symptomatic atrial fibrillation in a safe manner and with a high success rate. In addition, it is important to note that even in a patient population with frequently recurring atrial fibrillation that was refractory to pharmacological control, defibrillator efficacy was $90 \%$, which is considerably higher than pharmacologic therapy, and about $70 \%$ of the atrial fibrillation episodes were successfully treated with a single shock. Successful atrial defibrillation was associated with moderate discomfort, high patient satisfaction scores and no ventricular proarrhythmic events. These findings suggest that ambulatory use of an atrial defibrillator provide safe, successful and tolerable conversion of atrial fibrillation.

\section{ABSENCE OF PROARRHYTHMIA}

With correct R-wave synchronization and timing to assure that the shock is not delivered during the relarive refractory period of the ventricle, the risk of inducing malignant ventricular arrhythmias with low energy atrial defibrillation is small 6,7 . Although the mean left ventricular ejection fraction was normal, structural heart disease was present in a majority of the patients and no ventricular proarrhythmia 
was observed with delivery of more than 5000 shocks. Using the number of shocks delivered and the atrial fibrillation recurrence rate, the maximum proarrhythmic risk was less than $0.81 \% /$ year, far less than the proarrhythmic risk associated with any oral antiarrhythmic agent. The results of this study confirm the findings of other studies $^{6,7}$ that the risk of inducing a ventricular tachyarrhythmia as a result of atrial defibrillation is small and extends these findings to ambulatory therapy.

\section{PATIENT TOLERANCE OF SHOCK THERAPY}

In previous studies, patients undergoing internal defibrillation tolerated fewer high intensity shocks (3-6 J) better than mulciple low intensity shocks $(1-3)^{8,9}$. In this study, most atrial fibrillation episodes could be treared with a single shock, and most first shocks were programmed to maximum output. This high rate of single-shock success likely enhanced patient tolerance of defibrillation. There are other factors that may influence patient acceptance of shock therapy. Clinical, social and psychological factors such as severity of symptoms associated with atrial fibrillation, previous uncomfortable or painful experiences, surrounding stimuli, convenience of ambulatory and timely therapy may also effect patient tolerance. A recent study assessing shock discomfort in association with internal atrial defibrillation found that psychological components causing anxiety substantially contributed to the perception of discomfort ${ }^{10}$. In the present study, a questionnaire evaluating the impact of ambulatory therapy showed high patient satisfaction with only moderate discomfort.

\section{EARLY RECURRENCE OF ATRIAL FIBRILLATION}

Early recurrence of atrial fibrillation was likely responsible for most of the unsuccessful ambulatory cardioversions. Prior to ambulatory therapy, early recurrence of atrial fibrillation was observed by the physician during the treatment of 95 of the 388 episodes. Furthermore, prior to the device being programmed for ambulatory therapy, the physician could treat the early recurrence of arrial fibrillation with additional shocks and the administration of antiarthythmic drugs to obtain stable sinus rhythm. Although the device-stored electrograms occasionally captured early recurrence of atrial fibrillation during ambulatory therapy (Figure 3), its exact incidence could not be determined once the patient was treated away from the physician. Therefore, during ambulatory therapy, it was more difficult for the physician to detect and manage early recurrence of atrial fibrillation. The prevention of early recurrence of atrial fibrillation by oral administration of antiarrhythmic drugs 1,12 , by pacing techniques ${ }^{13}$ or by ablating the arrial fibrillation initiating arrial ectopic beat ${ }^{14}$ may increase the ambulatory efficacy while limiting the number of shocks required for episode termination. 
speed: $25 \mathrm{~mm} / \mathrm{s}$

RA-CS DIRECT: Sensitivity. $0.28 \mathrm{mV}$ Scale: $0.5 \mathrm{~mW} / \mathrm{cm}$

\section{RV DIRECT: \\ Sensitivity: \\ $4.5 \mathrm{mV}$ \\ Scale: \\ $5.0 \mathrm{mV} / \mathrm{cm}$}

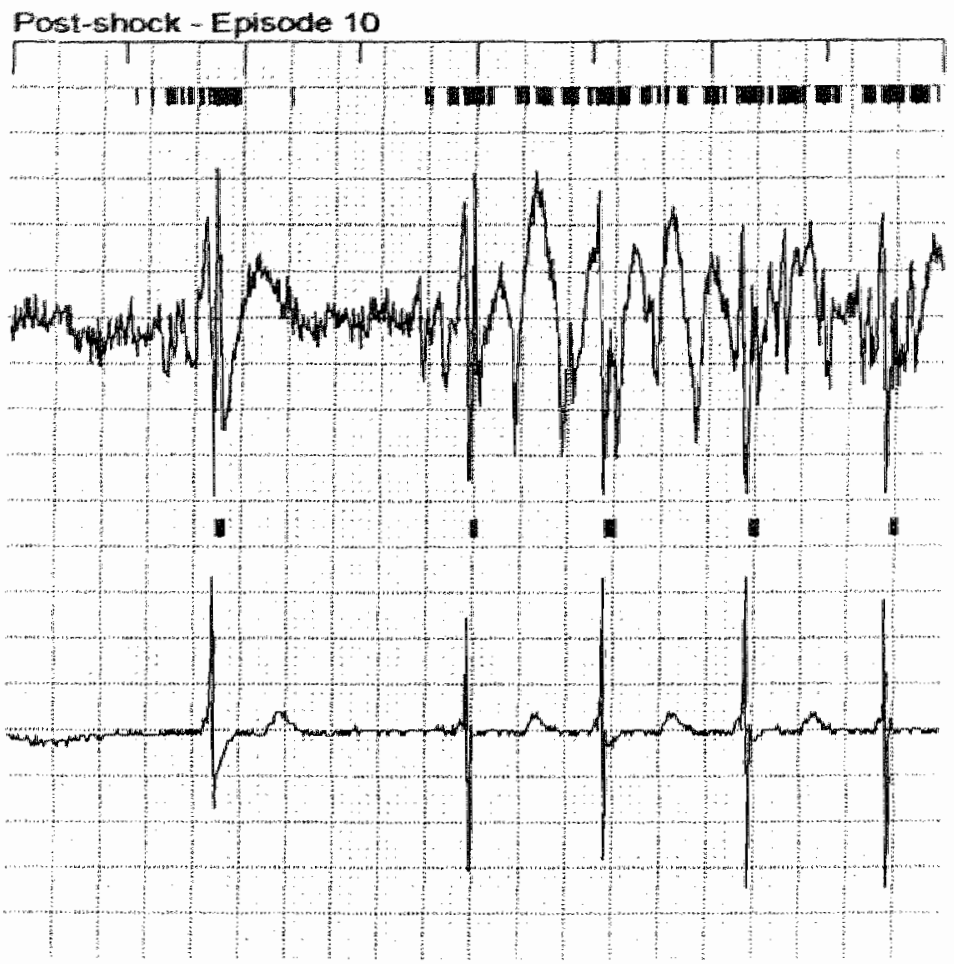

Figure 3. - Stoved edectrograms from the atrial defibrillator showing early recurrence of atrial fibrillaxion after a successful shock. "The upper electrogram is from the right arrium to coronary sinus detecrion/shock delivery vector and the lower electrogram is from the right ventricular bipolar vector.

\section{PREVIOUS STUDIES}

Studies of transvenous atrial defibrillation have demonstrated that a majority of parients with paroxysmal, persistent and permanent atrial fibrillation can be reliably cardioverted $2-4,8,11-14$. Recently, the efficacy of the atrial defibrillator was demonstrated in patients meeting strict implantation criteria ${ }^{5}$. In this initial study, atrial defibrillarion was performed under physician observation and the efficacy was similar to the results in this study. The current study, therefore, demonstrates that ambulatory therapy with the arrial defibrillator is efficacious and is not adversely affected by the absence of physician intervention.

\section{IMPACT OF AMBULATORY THERAPY}

It is important to speculate on the impact of out-of-hospital defibrillation on the cost of care and quality of life. Patients with frequent episodes of atrial fibrillarion, similar to rhe patients included in this study who had frequent contact with the 
health care system, often utilize the health care system at a significant cost ${ }^{15.16}$. In the current study, the efficacy of ambulatory therapy was high, likely resulting in fewer emergency room wisits and hospitalizations. Even for episodes in which ambulatory therapy was unsuccessful, the patient did not require hospitalization in order to restore sinus rhythm. Furthermore, as demonstrated in this study, cardioversion of atrial fibrillation relieved symptoms and thus may also improve parient's quality of life ${ }^{17}$. Further studies are needed to prospecrively evaluate the cost effecriveness of ambulatory atrial defibrillation with an implanted device.

\section{LIMITATIONS}

Antiarrhythmic therapy was not controlled in this study, therefore, no evaluation of its effect on atrial fibrillation could be made. However, despite antiarrhythmic therapy, patients continued to experience symptomatic atrial fibrillation. A second limitation is that these results are applicable only to patients with frequently recurring, drug-refractory, symptomatic, atrial fibrillation. Therefore, this population of patients may be more accepting of ambulatory shock therapy. A third limitation is that fewer than the total patients implanted transitioned to receive ambulatory therapy. As this is the initial experience with ambulatory defibrillation, it is likely that a greater percentage of patients will transition to receive ambulatory therapy in the future. Data pertaining to the reasons why parients did not transition were not collected in this study. Finally, due to the criteria for inclusion in this study, these results can not be extended to patients with depressed left ventricular ejection fraction or other conditions that may be associated with a higher risk of ventricular arrhythmias.

\section{CLINICAL IMPLICATIONS}

An implantable arrial defibrillator needs to satisfy several requirements to achieve therapeuric success in the ambulatory treatment of atrial fibrillation. The defibrillator must accurately detect atrial fibrillation, properly synchronize shocks and successfully cardiovert atrial fibrillation in a tolerable manner, and preferably controlled by the patient. This study confirms that the Metrix implanted atrial defibrillator is effective at detecting and terminating atrial fibrillation withour induction of ventricular proarrhythmia. Although patients perceived shock therapy as moderately uncomfortable, patient sarisfaction with the therapy was high. Whether an atrial defibrillator is cost-effecrive and improves quality-of-life remains to be determined. 


\section{REFERENCES}

1. Lown B, Amarasinghim R, Neaman J. New method for terminating cardiac arthythmias: use of synchronized capacitor discharge. JAMA 1962;182:548-555.

2. Murgarroyd FD, Slade AKB, Sopher SM, Rowland $\mathbb{E}$, Ward DE, Camm AJ. Efficacy and tolerability of unanswenous low energy cardioversion of paroxysmal arrial fibrillation in humans. J Am Coll Cardiol 1995:25:1347-1353.

3. Schmitt $C_{\text {, Alt }} \mathbb{E}_{\text {, Plewan }} \mathbb{A}_{\text {. Ammer }}$, Lebig M, Karch M, Schömig A. Low energy intracardiac cardioversion after falled conventional external cardioversion of atrial fibrillation. I Am Coll Cundiol 1996:28:994-999.

4. Levy S, Ricard P, Lau CP, Lok NS, Camm AJ, Murgatroyd FO, Jordaens L, Kappenberger LI, Bugada , Ripley KL. Multicenter low energy trantvenous atrial defibrillation (XAD) rrial results in cifferene subsece of atrial fibrillation. J Am Coll Cardiol 1997:29:750-755.

5. Wellens HIJ), La CP, Lüderiz B, Akhar M, Waldo AM, Camm AJ, Timmermans C, Tse HF, Jung $W$, Jordaens $L$, Ayers $G$, for the Merrix Investigators. The atrial defibrillatot: an implantable device for the rreatment of atrial fibrillation. Circulation 1998;98:1651-1656.

6. Tse HF, Lau CP, Sra JS, Crijns HJGM, Edvardsson N, Kacer $S$, Wyse G, for the Metrix Investigators. Atrial fibrillation detection and R-wave synchronization by Metrix implantable atrial defibrillator: implications for long-term efficacy and safety. Circulation 1999:99:1446-1451.

7. Ayers GM, Alferness CA, Wagner DO, llina MI, Sirokman WA, Adams JM, and Griffin JC. Effect of ventricular cycle length and shock strength on ventricular vulnerability in a sheep model of transwenous arrial defibrillation. Circulation 1994:89:413-422.

8. Lok NS, Lau CP. Tse HF, Ayers GM. Clinical shock tolerability and effect of different right atrial electrode locations on efficacy of low energy human transvenous atrial defibrillation using an implantable lead system. J Am Coll Cardiol 1997;30:1324-1330.

9. Ayers GM. How can atrial defibrillation be made more tolerable. In: Nonmpharmacological management of atrial fibrillation. Edited by A. J. Camm and F. Murgatroyd, Armonk, NY: Futura Publishing Company, Inc; 1997:475-487.

10. Timmermans C, Rodrigue L.M. Ayers GM, Lambert H, Sneets JLMR, Vlacyen JWS, Albert A, Wellens HJJ. Effer of bucorphanol tartrate on shock-related discomfort during internal atrial defibrillation. Circulation 1999;99:1837-1842.

11. Jimmermans C, Rodriguez LM, Sneets JRLM, Wellens HJJ. Immediate reinitiation of atrial fibrillation following internal atrial defibrillation. J Cardiovasc Electrophysiol 1998;9:122-128.

12. Boriani G. Biffi M, Capucci A, Bronzeti G. Ayers GM, Zannoli R, Branzi A, Magnani B. Favorable effects of flecainide in transwenous internal cardioversion of atrial fibrillarion. I Am Coll Cardiol $1999,33,333-341$

13. Tse HF, Lau CP. Ayers GM. Atrial overdrive pacing for suppression of spontaneous early re-intiation of arrial fibrillacion after transvenous atrial defibrillacion. I Am Coll Cardiol $1999: 33: 147 \mathrm{~A}$.

14. Lau CP", Tse HF, Ayers GM. Defibrillation - guided radiofrequency ablation of atrial fibrillation secondary to an atrial focus. I Am Coll Cardiol 1999;33:1217-26. 
15. Maglio C, Sra I, Paquerte M, Dorian P. Bygrave A, Wood K. Ayers GM. Measuring quality of life and symptom severity in patients with atrial hbrillation. Pacing Clin Electophysiol 1998:21:839.

16. Bialy D, Lehrman MH, Schumaker DN, Steinman RT, Meissner MD. Hospitalization for arrhythmias in the Unired Stares; Importance of atrial fibrillation. J Am Coll Cardiol 1992;19:41 A.

17. Jung W, Luderizz. B. Quality of life in patients with atrial fibrillation. J Cardovasc Electrophysiol $1998 ; 9: 5177-5186$

\section{APPENDIX}

\section{LIST OF PARTICIPATING CENTERS AND INVESTIGATORS}

Allegheny University Hospitals, Philadellphia, PA (D. Callans, J. Fontaine, C. Gottlieb, S. Hessen, S. Kutalek, F. Marchlinski, C. Mowsowizz: F. Samuels, S. Wilbur); Baylor Hospiral, Dallas, TX (J. Franklin, P. Coffeen, K. Wheelan, J. Shinbane, T. Beveridge, J. Hurvitz, P. Wells, C. East); Beth Isael Medical Center, Boston, MA, (P. Zimerbaum, L. Epsrein, M. Josephson, P. Papageorgiou); Scripts Memorial Hospical, La Jolla, CA, (S. Higgins, D. Meyer); Sacred Heare Hospital; Eugene, OR (M. Ancimisiaris, J. McClelland); Cleveland Clinic, Cleveland, OH B. (Wilkoff, M. Niebauer, F. Jeager, M. Chung, G. Kidwell, P. Tchou, L. Castle, R. Augosrini); Duke Uniwersity Medical Center, Durham, NC (JM Wharton, T Bahnson, R. Sorrentino, R. Greenfield); Emory Universiry, Arlanta, GA (A. Leon, I. Langberg, D. Lurgio); Genesis Medical Center, Davenporr, LA (M. Guidici, D. Orias, K. VanWhy); Good Samaritan Hospital, Los Angeles, CA (D. Cannom, C. Prejean, R. Marthews, R. Leman, N. Hunter, G. Kay, M. Estioki, A. Bhandari, E. Abdullah); Iowa Heart Center, Des Moines, $\mathbb{L A}\left(\mathbb{R} . H_{0}\right.$ oyt, WB Johnson, S. Bailin); Loyola University Medical Center, Maywood, LL (B. Olshansky, R. Kawasaki, R. Picchi-Szocka); Massachuserrs General Hospital, Boston, MA (H. Garan, C. Albert, P. O'Callaghan, C. Carpenter, J. Galvin, D. Keane, B. McGovern, M. Orlov); McGuive VA Hospital, Richmond, VA (R. Shepard, M. Wood, D. Gilligan, G. Fu, D. Dan, H. Clemo); Riverside Methodist Hospital, Columbus, OH (E. Daoud, J. Hummel, S. Kalbfleisch); Rhode Island Hospiral, Providence, RI (R. Lemeny): University of Kentucky, Lexington, KY (A. Natale, F. Leonellix G. Tomassoni); Universiry of Chicago, Chicago, IL (D. Wilber, R. Verdino, D. Kopp, J. Kall M. Burke); Universiry of Maryland, Balcimote, MD (M. Gold, M. Olsovsky); Universiry of Michigan, Am Arbor, MI (A. Strickberger, F. Morady, R. Goyal, M. Harwey, K. Ching Man); University of Utall Medical Center, Salt Lake, UTC (R. Freedman, R. Klein, J. Mason, G. Muelheims, P. Spector, S. Compton); Virginia Mason, Seattle, WA (C. Fellows, K. Foley, D. Landreth. R. Stewart, J. Smith, R. Vielbig); West LA VA Medicall Center, Los Angeles, CA (P. Sager, M. Bersohn); Sr. Luke's Hospital, Milwaukec, WI O. Sra, M. Akhtar, M. Miehl, Z Blanck, S. Deshpande, A. Dhala, H. Jazayeri, D. Kress); University Hospitals of Cleveland, Cleveland, OH (L. Biblo, J Mackall, A. Waldo, D. Rosenbaum, M. Cartson); Academic Hospiral Maastricht, Maastricht, the Netherlands (H. Wellens, C. Timmermans, L.M. Rodriguet, P. Barenbrugi. 



\section{Summary}

Antiarrhythmic drugs and external defibrillation are presently the primary modes of treatment of patients with atrial fibrillation. Due to the limited efficacy and safery of antiarthythmic drugs and the repeared hospitalizations for external defibrillation requiring general anesthesia, several non-pharmacologic options have been developed, especially for patients with recurrent atrial fibrillation. These options include pacemaker therapy, radiofrequency catheter ablation or modification of the AV junction with or withour pacemaker implantation, radiofrequency catherer ablation of "focal" atrial fibrillation, surgical or catheter-based compartmentalization of the atria, internal atrial defibrillation and an implantable atrial defibrillator. This thesis focuses on recent progress in the electrical treatment of atrial fibrillation. The first part of the study (Chapters 2 to 5) describes new observations in internal atrial defibrillation, while the second part (Chapters 6 to 11 ) reports on the first experience with an implantable atrial defibrillator.

In Chapter 2, the effect of electrode length on atrial defibrillation thresholds is evaluated in 15 patients. Two decapolar catheters were inserted, one into the distal coronary sinus and one in the right atrium. To provide longer electrode lengths, a third catheter was inserted and alternatingly positioned in the right atrium or coronary sinus. A $6 \mathrm{~cm}$ electrode span was obtained by using the distal 8 rings on the coronary sinus catheter or 8 consecutive rings on the right atrial catheter. The electrode span was increased from 6 to $11 \mathrm{~cm}$ by connecting 5 consecutive, non-overlapping rings of the third catheter with the 10 rings of the initial right atrial or coronary sinus catheter. Atrial defibrillation thresholds were determined twice, in a randomized order, in each patient for each of the three combinations of electrode lengths. All parients were successfully cardioverted. Mean thresholds were $306 \pm 102$ $V, 5.9 \pm 4.0 \mathrm{~J}$ for the $6 \mathrm{~cm} / 6 \mathrm{~cm}$ electrode length combination with an impedance of $72 \pm 18 \Omega$. For the electrode combination using the $11 \mathrm{~cm}$ electrode in the right atrium, the defibrillation threshold was $296 \pm 107 \mathrm{~V}, 5.8 \pm 3.9 \mathrm{~J}$ with an impedance of $61 \pm 17 \Omega$. It was $294 \pm 91 \mathrm{~V}, 5.6 \pm 3.6 \mathrm{~J}$ with an impedance of $55 \pm 11 \Omega$ for the $11 \mathrm{~cm}$ electrode in the coronary sinus. There were no significant differences in defibrillarion voltage or energy $(p>0.05)$ associated with the longer electrodle lengths; however, the longer electrode lengths did significantly lower shock impedance $(\mathrm{p}<0.05)$.

In Chapter 3 , the number of leads required for an implantable arrial defibrillator is investigated. In 16 patients undergoing elective cardioversion of atrial fibrillation, a 2-lead system was compared to a 3 -lead system in a randomized trial. The new 2-lead system consisted of a catheter with a distal bipolar right ventricular electrode pair and a proximal right atrial shock electrode coil and a separate decapolar 
defibrillation catheter in the coronary sinus. For the 3-lead system, the proximal right atrial shock electrode coil was disconnected and replaced by a second decapolar defibrillation catheter. The defibrillation coil or catheters in the coronary sinus and right atrium were used for detection of atrial fibrillation and for shock delivery. The bipolar right ventricular electrode pair was used for synchronization and post-shock pacing. Atrial fibrillation and sinus rhythm signal amplitude detection and atrial defibrillation threshold were compared in each patient using both systems. Successful defibrillation was obtained in all patients. The defibrillation threshold for the 2-lead system (mean $370 \pm 112 \mathrm{~V}, 9.3 \pm 5.2 \mathrm{~J}$ ) was significantly higher compared to the 3-lead system (mean $316 \pm 100 \mathrm{~V}, 6.8 \pm 4.2 \mathrm{~J} ; \mathrm{p}<0.05$ ). In contrast, there was an increase in impedance for the 3-lead system $(77 \pm 16 \Omega$ vs $68 \pm 13 \Omega, \mathrm{p}<0.05)$. The mean right atrium-coronary sinus signal amplitude was $1.7 \pm 0.8 \mathrm{mV}$ during sinus rhythm and $0.86 \pm 0.17 \mathrm{mV}$ during atrial fibrillation in the 2-lead system. For the 3-lead system, the right atrium-coronary sinus signal amplitude was $2.54 \pm 1.07$ $\mathrm{mV}$ and $1.59 \pm 0.53 \mathrm{mV}$ respectively. Atrial fibrillation had a lower signal amplitude as compared to sinus thythm for both systems $(p<0.05)$ and the 2-lead system had a lower signal amplitude as compared to the 3-lead system for both rhythms $(p<0.05)$. The use of a 2-lead system with this configuration is inferior to the 3-lead system with respect to atrial fibrillation signal amplitude detection and atrial defibrillation threshold.

In Chapter 4, the effect of intranasal butorphanol tartrate, an opioid, on shock-related discomfort during internal atrial defibrillation is described. In a double-blind, placebo-controlled manner, the effect of transnasally administrated butorphanol was evaluated in 47 parients with the use of a step-up internal atrial defibrillation protocol (stage I). On request, additional butorphanol was administrated and the step-up protocol continued (stage II). Thereafter, if necessary, patients were intravenously sedated (stage III). After each shock, the McGill Pain Questionnaire was used to obtain a sensory (S), affective (A), evaluative (E), and total (T) pain raring index (PRI) and a visual analogue scale analyzing pain (VAS-P) and fear (VAS-F). For every patient, the slope of each pain or fear parameter against the shock number was calculated and individual slopes were averaged for the placebo and butorphanol group. All patients were cardioverted at a mean threshold of $4.4 \pm$ $3.3 \mathrm{~J}$. Comparing both patient groups for stage II, the mean slopes for PRI-T $(p=0.0099), \mathbb{P R I}-S(p=0.019)$, and PRI-E $(p=0.015)$ became significantly lower in the butorphanol group than in the placebo group. Comparing patients who received the same shock intensity ending stage I and going to stage II, in those parients randomized to placebo the mean VAS-P $(p=0.023)$, PRI-T $(p=0.029)$, PRI-S $(p=0.030)$, and PRI-E $(p=0.023)$ became significantly lower after butorphanol administration. The results show that during a step-up internal atrial defibrillation protocol, intranasal butorphanol decreased or stabilized the value of several pain variables and did not affect fear. Of the 3 qualitative components of pain, only the 
affective component was not influenced by butorphanol. The PRI evaluated pain more accurately than the VAS.

In Chapter 5, the intriguing phenomenon of immediate reinitiation of atrial fibrillation (IRAF) following internal atrial defibrillation is analyzed. Thirty-eight patients underwent internal atrial defibrillation. Catheter-based defibrillation electrodes were positioned in the anterolateral right atrium and the coronary sinus. All patients were cardioverted at a mean threshold of $4.6 \pm 3.4 \mathrm{~J}$. Five of 38 patients $(13 \%)$ had 1 to 4 episodes of $\mathbb{R A F}$. No differences in clinical and echocardiographic characteristics were observed when patients with and without IRAF were compared. Atrial fibrillation was always reinitiated by an atrial premature bear. When the earliest atrial endocardial activation time on the defibrillation catheters was analyzed, these atrial premature beats did not seem to originate from the defibrillation catheters. Twenty-one patients had atrial premature beats without IRAF. When the coupling intervals of the first atrial premature beat in patients without and with IRAF after conversion were compared, a significant difference was found (661 \pm 229 vs $418 \pm 79 \mathrm{~ms}, \mathrm{p}<0.05$ ). IRAF was successfully treated with repeated shock delivery after the administration of atropine in 1 patient and intravenous flecainide in 2 patients. Only repeated shock delivery was sufficient to treat IRAF in another 2 patients. IRAF did not predict early recurrences of the arrhythmia after discharge from the hospital, emphasizing the necessity to treat immediate reinitiation promptly to achieve a longer lasting successful cardioversion.

In Chapter 6, the first experience with an implantable atrial defibrillator (the Atrioverter) for the treatment of atrial fibrillation is described. The Atrioverter, a pectorally implanted device, is connecred to right atrial and coronary sinus defibrillation leads and a bipolar endocardial ventricular pacing lead. In a prospective worldwide multicenter study, the safety and efficacy of the Atrioverter was evaluated in 51 patients with recurrent symptomatic atrial fibrillation who had not responded to antiarrhythmic drugs. Most of these patients had no structural heart disease and a normal left ventricular function. In none of the patients there was evidence of ventricular arrhythmias. The atrial fibrillarion detection and $\mathbb{R}$-wave synchronization algorithms were tested at implantation and during follow-up. Shock termination of spontaneous episodes of atrial fibrillation was always performed under physician observation. During a follow-up of 72 to 613 days (mean, $259 \pm$ 138 days), $96 \%$ of 227 spontaneous episodes of atrial fibrillation in 41 parients were successfully converted to sinus thythm by the Atrioverter. In $27 \%$ of episodes, several shocks were required because of IRAF. There were no cases of induction of ventricular arrhythmias or inaccurately synchronized shocks during the study. Analysis of the atrial fibrillation detection algorithm performance revealed a $100 \%$ specificity for the recognition of sinus rhythm and $92.3 \%$ sensitivity for the derection of atrial fibrillation. Most patients received antiarrhythmic medication during follow-up. In 4 parients, the Atrioverter was removed: in 2 because of infection, in 1 because of cardiac tamponade, and 1 because of frequent episodes of atrial fibrilla- 
tion requiring His bundle ablation. With the Atroverter, prompt and safe restoration of sinus rhythm is possible in patients with recurrent atrial fibrillation.

In Chapter 7, the spontaneous episodes of atrial fibrillation after implantation of the Arrioverter and the effect of repeated defibrillation on the clinical course of the arrhythmia are analyzed in the same patient population as described in Chapter 6. Monitoring of the cardiac rhythm was performed by the device on periodic intervals. During a mean follow-up of $260 \pm 144$ days, 1161 episodes of atrial fibrillation were observed in 45 of the 51 parients. Forty-one patients experienced 231 episodes for which they sought defibrillation therapy. The average duration of the treated episodes was significantly longer than the nontreated episodes (38 44 vs $10 \pm 8$ hours; $\mathrm{p}<0.05)$. The time between episodes requiring Atrioverter therapy lengthened and the risk of having an episode requiring treatment decreased. No changes were observed in the number and duration of the short lasting, nontreated episodes as time since implant of the device increased.

In Chapter 8, the question. whether the electrophysiological changes induced by longer lasting atrial fibrillation are rewersible in patients prompty defibrillated for their atrial fibrillation episodes with the Atrioverter was addressed. Four patients with recurrent atrial fibrillation (duration, 3 to 21 years, mean $13 \pm 7.6$ years) were studied after the implantation of an Atrioverter. The Atrioverter stores and analyzes 3 minutes of cardiac rhythm every hour. Before implantation, the atrial effective refractory period (AERP) was measured. During a mean follow-up of 14 months, 52 spontaneous (39 treated and 18 nontreated) atrial fibrillation episodes occurred while the patients were on antiarrhythmic drugs. All parients were electrophysiologically studied after they had been in sinus rhythm for at least 1000 hours (range, 1052 to 2675 hours). Before Arrioverter implantation, atrial fibrillation was induced by 1 atrial premature beat in 3 patients and not unduced in the remaining parient. After a long period of sinus thythm ( $>1000$ hours), atrial fibrillarion could be induced in the same 3 patients in the same way as before implantation. In the patient in whom no atrial fibrillation was induced, right AERP values measured using the single extrastimulus techmique at 3 pacing cycle lengths (600, 500 and $430 \mathrm{~ms}$ ) were similar to those before implantation. We concluded that in the studied parients with longer lasting recurrent atrial fibrillation, no return to "notmal" atrial electrophysiology could be demonstrated.

In Chapter 9, a parient with atrial arrhythmias secondary to severe congenital heart disease successfully and safely treated with the Atrioverter is reported.

In Chapter 10, rhe use of sedation during cardioversion with the Atrioverter is analyzed in 12 patients. During the in hospital treatment of atrial fibrillation episodes, intravenous sedation was given only upon patient request. Efficacy, number of shocks delivered and sedation requirements were recorded. A total of 393 shocks ( $1.8 \pm 1.6$ shocks/atrial fibrillation episode) were delivered to treat 213 atrial fibrillation episodes; 85 of 213 atrial fibrillation episodes (40\%) were treated away from the hospital. Sinus rhythm was restored in 195 episodes (92\%). Five patients 
never requested sedation. No sedation was needed for ambulatory-treated atrial fibrillation episodes. During the treatment of 26 of 213 atrial fibrillation episodes $(12 \%), 75$ shocks were delivered after parients received sedation. The number of shocks required to treat an atrial fibrillation episode determined the need for sedation $(2.9 \pm 2.7$ shocks for atrial fibrillation episodes requiring sedation ws $1.7 \pm 1.3$ shocks for atrial fbrillation episodes requiring no sedarion; $p<0.001$ ). These additional shocks were needed to treat IRAF (14 episodes) or initial failure to cardiovert ( 4 episodes). For 8 atrial fibrillation episodes, sedarion was requested before to the first shock. This study suggest that, in a selected group of patients, atrial fibrillation can be treated with Arriovercer therapy without sedation. Successful ambulatory rreatment of atrial fibrillation episodes with the Atrioverter, programmed to deliver $\leq 2$ shocks, did not require sedation. When multiple shocks were required to rreat an atrial fibrillation episode, the need for sedation increased and included patients inirially not requesting sedation.

In Chapter 11, the initial experience with the ambulatory use of the Arrioverter is described. This study included 105 patients. For at least the first 3 months after implant, the device was programmed to a monitoring mode with episodes of atrial fibrillation defibrillated in the presence of a physician. Thereafter, physicians were allowed to program the device for either patient-activated or automatic therapy. Patients were asked to complete a questionnaire that assess severity of symptoms, therapy discomfort and satisfaction with therapy using a 10-point visual-analogue scale after each treated atrial fibrillation episode. Of the 105 patients, 48 reached at least 3 months follow-up and met the transition criteria. The 48 parients received therapy for 275 episodes with a therapy efficacy of $90 \%$ for which an average of $1.6 \pm$ 1.2 shocks per episode were delivered (median 1). For successful therapy, parients rated the discomfort as $5.2 \pm 2.4$ and $4.2 \pm 2.2$ for unsuccessful therapy ( $>0.05$ ). The satisfaction score was lower for unsuccessful as compared to successful therapy (3.4 \pm 3.3 w $8.7 \pm 1.3, p<0.05)$. Also in this study, shocks did not induce ventricular arrhythmias. The first experience with the ambulatory use of the Arroverter shows that most episodes of atrial fibrillation can be safely and successfully treated. Successful atrial deforillator treatment of atrial fibrillation is associared with high therapy sarisfaction and only moderate shock discomfort. 



\section{Samenvatting}

Anti-aritmica en uitwendige defibrillatie vormen regenwoordig de hoeksteen bij de behandeling van patiënten met boezemfibrilleren. Omdat anti-aritmica slechts een beperkte effectiviteit hebben en hun inname met complicaties gepaard kan gaan en ook omdat uitwendige defibrillatie, zeker voor patiënten met herhaalde episoden van boezemfibrilleren, frequente ziekenhuisopnamen en algehele anesthesie vereist, werden verscheidene niet-medicamenteuze behandelingen ontwikkeld. Deze niet-medicamenteuze behandelingen bestaan uit pacemaker implantatie, katheterablatie of modificatie van de geleiding in de AV-knoop met behulp van radiofrequente energie, al dan niet gevolgd door pacemaker implantatie, katheterablatie van "focaal" boezemfibrilleren, het in compartimenten verdelen van de arria met chirurgische of kathetertechnieken, inwendige boezemdefibrillatie en de implanteerbare boezemdefibrillator. Dit proefschrift handelt over recente ontwikkelingen in de elektrische behandeling van boezemfibrilleren. In het eerste deel van het onderzoek (Hoofdstukken 2 tot 5) worden nieuwe observaries bij inwendige boezemdefibrillatie beschreven en in her tweede deel (Hoofdstukken 6 tot 11) worden de eerste ervaringen met de implanteerbare boezemdefibrillator gerapporteerd.

In Hoofdstuk 2 wordt het effect van de elektrodelengte op de boezemdefibrillatie drempel nagegaan bij 15 patiënten. Een 10-polige katheter werd zowel in de distale sinus coronarius als in de rechter boezem gepositioneerd. Een langere elektrodelengte werd bereikt door een derde katherer afwisselend in de sinus coronarius en de rechter boezem te positioneren. Een elektrodelengte van $6 \mathrm{~cm}$ werd verkregen door gebruik te maken van de distale 8 ringen van de sinus coronarius elektrode of 8 opeenvolgende ringen van de rechter boezem katherer. Een elektrodelengte van 11 $\mathrm{cm}$ werd verkregen door 5 consecutieve ringen van de derde katheter met de 10 ringen van de rechter boezem of sinus coronarius katherer te verbinden. Bij elke patiënt werden boezemdefibrillatie drempels tweemaal op een gerandomizeerde wijze bepaald voor elk van de 3 elektrodelengte combinaties. Alle patiënten werden succeswol gecardioverteerd. De gemiddelde drempels waren $306 \pm 102 \mathrm{~V}, 5.9 \pm 4.0 \mathrm{~J}$ met een impedantie van $72 \pm 18 \Omega$ voor de $6 \mathrm{~cm} / 6 \mathrm{~cm}$ elektrodelengte combinatie. Voor die combinatie waar een $11 \mathrm{~cm}$ elektrode in de rechter boezem werd gebruikt, was de defibrillatie drempel $296 \pm 107 \mathrm{~V}, 5.8 \pm 3.9 \mathrm{~J}$ met een impedantie van $61 \pm$ $17 \Omega$. De drempel bedroeg $294 \pm 91 \mathrm{~V}, 5.6 \pm 3.6 \mathrm{~J}$ met een impedantie van of $55 \pm$ $11 \Omega$ voor de $11 \mathrm{~cm}$ elektrode in de sinus coronarius. Er was geen significant verschil in defibrillatie voltage of energie ( $p>0.05$ ) wanneer een langere elektrodelengte werd gebruikt. Niettemin was de impedantie significant lager met deze langere elektroden $(\mathrm{p}<0.05)$. 
$\left.138\right|^{\text {Sannenwaring }}$

In Hoofdstuk 3 werd her antal leads die nodig zijn voor een implanteerbare boezemdefibrillator geévalueerd. Bij 16 patiénten die een electieve cardioversie van boezemfibrilleren ondergingen, werd op een gerandomizeerde wijze een 2 -lead systeem met cen 3-lead systeem vergeleken. Het nieuwe 2-lead systeem bestond uit een karheter met distaal een rechter kamer elektrodepaar en proximaal een rechter boezem schokelektrode coil en een 10-polige defibrillatie katherer in de sinus coronarius. Voor het 3-lead systeem werd de proximale rechter boezem schokelektrode coil losgekoppeld en vervangen door een tweede 10-polige defibrillatie katheter. De defibrillatie coil of katheters in de sinus coronarius en de rechter boezem werden gebruikt voor de detectie van boezemfibrilleren en voor het geven van een schok. Het bipolaire rechter ventrikel clektrodepaar werd gebruikt voor synchronisatie en post-schok pacing. Bij elke patiënt werd voor beide lead systemen, de amplitude van de signalen zowel tijdens boezemfibrilleren als tijdens sinusritme en ook de boezemdefibrillatie drempel vergeleken. Alle patiënten werden succeswol gedefibrilleerd. De defibrillatie drempel verkregen met her 2 -lead systeem $(370 \pm 112 \mathrm{~V}, 9.3 \pm 5.2 \mathrm{~J})$ was significant hoger in vergelijking met het 3 -lead systeem $(316 \pm 1.00 \mathrm{~V}, 6.8 \pm 4.2 \mathrm{~J}$; $\mathrm{p}<0.05)$. De impedantie van het 3 -lead systeem was echter hoger $(77 \pm 16 \Omega$ vs $68 \pm$ $13 \Omega, \mathrm{p}<0.05$ ). De gemiddelde rechter boezem-sinus coronarius signaalamplitude was $1.7 \pm 0.8 \mathrm{mV}$ tijdens sinusritme en $0.86 \pm 0.17 \mathrm{mV}$ tijdens boezemfibrilleren voor het 2-lead systeem. Voor het 3-lead systeem bedroeg de rechter boezem-sinus coronarius signaalamplitude respectievelijk $2.54 \pm 1.07 \mathrm{mV}$ en $1.59 \pm 0.53 \mathrm{mV}$. Boezemfibrilleren kenmerkte zich door een lagere signaalamplitude dan sinusritme en dit gold voor beide systemen $(\mathrm{p}<0.05)$. Het 2 -lead systeem registreerde een lagere signaalamplitude in vergelijking met het 3 -lead systeem en dit gold voor beide ritmen $(\mathrm{p}<0.05)$. Een 2 -lead systeem met deze configuratie is dus minder efficiënt dan een 3-lead systeem met betrekking tot de detectie van de signaalamplitude van boezemfibrilleren en de boezemdefibrillatie drempel.

In Hoofdstuk 4 wordt het effect van intranasal toegediend butorphanol tartrate, een opiaat, beschreven op het aan een schok gerelateerd ongemak tijdens inwendige boezemdefibrillatie. Op een double-blind, placebo-gecontroleerde wijze werd het effect van transnasaal toegediend butorphanol onderzocht bij 47 patiënten tijdens een "step-up" inwendig boezemdefibrillatie protocol (stadium I). Op verzoek werd opnieuw butorphanol toegediend, en werd het defibrillatieprotocol voortgezet (stadium II). Zonodig werden de patiënten intraveneus gesedeerd (stadium III). Een pijnspecifieke vragenlijst, de McGill Pain Questionnaire, en een visueel analoge schaal (VAS) werd na elke schok ingevuld. Met de vragenlijst werd een sensoriele (S), affectieve (A), evaluatieve (E) en een totale (T) pijn index (PRI) berekend. Een visueel analoge schaal werd zowel voor de analyse van pijn (VAS-P) als voor de analyse van angst (VAS-F) gebruikt. Voor elke patiënt werd de hellingshoek van elke pijn of angst parameter ten opzichte van het aantal schokken berekend. Van deze individuele hellingshoeken werd zowel voor de placebo als voor de butorphanol groep het gemiddelde berekend. De gemiddelde cardioversie drempel bedroeg $4.4 \pm$ 
3.3 J. Toen beide patiënten groepen vergeleken werden rijdens stadium II, bleek de gemiddelde hellingshoek van PRI-T $(p=0.0099)$, PRI-S $(p=0.019)$, en PRI-E $(\mathrm{p}=0.015)$ significant lager in de butorphanol groep dan in de placebo groep. Toen de patiënten die gedefibrilleerd met dezelfde schok intensiteit rijdens de overgang van stadium I naar stadium II vergeleken werden, bleek dat bij de pariënten van de placebo groep de gemiddelde waarde van VAS-P $(\mathrm{p}=0.023)$, PRI-T ( $\mathrm{p}=0.029)$, PRI-S ( $p=0.030)$, en PRI-E ( $p=0.023)$ significant afnam na toediening van butorphanol. Deze gegevens tonen aan dar rijdens een "step-up" inwendig boezemdefibrillatieprotocol, intranasaal toegediend butorphanol de waarde van de geanalyseerde pijnvariabelen verminderde of stabiliseerde en geen effect had op angst. Van de 3 kwalitatieve pijncomponenten, werd enkel de affectieve component niet benvloed door butorphanol. De PRI evalueerde pijn nauwkeuriger dan de VAS.

In Hoofdstuk 5 wordt de onmiddellijke reïntiatie van boezemfibrilleren na een geslaagde inwendige boezemdefibrillatie beschreven. Bij 38 patiënten werd een inwendige cardioversie verricht. Defibrillatie elektroden werden in het anterollaterale deel van de rechter boezem en in de sinus coronarius gepositioneerd. Alle patiënten werden gecardioverteerd met een gemiddelde drempel van $4.6 \pm 3.4 \mathrm{~J}$. Vijf van de 38 patiënten (13\%) hadden 1 tot 4 episoden van onmiddellijke reïnitiatie van boezemfibrilleren. Tussen de patiënten met of zonder onmiddellijke reïnitiatie van boezemfibrilleren waren er geen verschillen in klinische en echocardiografische karakteristieken. Boezemfibrilleren trad steeds opnieuw op na een boezemextrasystole. Na analyse van de vroegste endocardiale activatierijd bleken deze boezemextrasystolen niet afkomstig te zijn van de defibrillarie katheters. Boezemextrasystolen kwamen voor bij 21 patiënten die geen onmiddellijke reïnitiatie van boezemfibrilleren hadden. De gemiddelde koppelingsinterval van de eerste boezemextrasystole bij patiënten met of zonder onmiddellijke reïnitiatie van boezemfibrilleren was significant verschillend $(418 \pm 79 \mathrm{~ms}$ vs $661 \pm 229, \mathrm{p}<0.05)$. Onmiddellijke rë̈nitiatie van boezemfibrilleren werd voorkomen door cen tweede defibrillatieschok na de intraveneuze toediening van flecainide ( 2 patiënten) of atropine (1 patiënt). Bij de overige 2 pariënten trad er na een volgende defibrillatieschok geen onmiddellijke reïnitiatie van boezemfibrilleren meer op. De onmiddellijke reïnitiatie van boezemfibrilleren ging niet samen met het optreden van een snel recidief van de ritmestoom is na ontslag uit het ziekenhuis. Een gerichte behandeling van dit probleem bewerkstelligt dus het langdurig succes van een cardioversie.

In Hoofdstuk 6 wordt de eerste ervaring met een implanteerbare boexemdefibrillator (de Atrioverter) voor de behandeling van boezemfibrilleren beschreven. De Atrioverter wordt pectoraal geïmplanteerd en verbonden mer een rechter boezem en een sinus coronarius defibrillatie lead, en een bipolaire rechter kamer stimulatie lead. In een prospectieve, wereldwijde multicentrum studie werd de veiligheid en de effectiviteit van de Atrioverter bij 51 patiënten met herhaalde symptomatische episoden van boezemfibrilleren geëvalueerd. Bij deze pariënten waren anti-aritmica ineffectief voor de behandeling van de ritmestoornis. De meeste patiënten hadden geen struc- 
turele hartafwijkingen en een normale linker kamerfunctie. Bij geen van hen waren er aanwijzingen woor de aanwezigheid van kamer ritmestoornissen. Het boezemfibrillarie derectie algoritme en het $\mathrm{R}$-golf synchronisatie algoritme werden geëvalueerd tijdens de implantatie en gedurende de follow-up. Defibrillatie van een spontane boezemfibrillatie episode gebeurde steeds in aanwezigheid van een arts. Tijdens de follow-up periode, die varieerde tussen 72 en 613 dagen $(259 \pm 138$ dagen), werden bij 41 patiënten $96 \%$ van de 227 spontane episoden van boezemfibrilleren succesvol geconverteerd tot sinusritme met de Atrioverter. Bij 27\% van de episoden werden verscheidene schokken toegediend omwille van de onmiddellijke reïnitiatie van boezemfibrilleren na een geslaagde defibrillatie. Tijdens de studie werden geen kamer ritmestoornissen geïnduceerd en werden er geen onnauwkeurig gesynchroniseerde schokken toegediend. Het boezemfibrillatie detectie algoritme was $100 \%$ specifiek voor de herkenning van sinusritme en $92.3 \%$ sensitief voor de detectie van boezemfibrilleren. Tijdens de studie werden de meeste patiënten behandeld met anti-aritmica. De Atrioverter werd verwijderd bij 4 patiënten omwille van infectie ( 2 patiënten), harttamponade ( 1 patiënt) en te frequente episoden van boezemfibrilleren waarvoor een His bundel ablatie werd verricht (1 patiënt). Het bleek dus dat bij patiënten met herhaalde episoden van boezemfibrilleren de Atrioverter snel en veilig het sinusritme kan herstellen.

In Hoofdstuk 7 werden analyses verricht naar de spontane episoden van boezemfibrilleren nadat de Atrioverter was geimplanteerd en naar het effect van het herhaald defibrilleren op het klinisch verloop van de ritmestoornis bij dezelfde patiënten populatie beschreven in Hoofdstuk 6. Met regelmatige tijdsintervallen werd het hartritme door het toestel geanalyseerd. Tijdens een gemiddelde follow-up van 260 \pm 144 dagen, traden 1161 episoden van boezemfibrilleren op bij 45 van de 51 patiënten. Bij 41 patiënten werden, op hun verzoek, 231 episoden met de Atrioverter behandeld. De gemiddelde duur van de behandelde episoden was significant langer dan van de niet-behandelde episoden ( $38 \pm 44$ vs $10 \pm 8$ uur; $p<0.05$ ). Het tijdsinterval tussen de behandelde episoden nam toe en het risico op een te behandelen episode nam af. Het aantal en de duur van de kortdurende, niet-behandelde episoden was niet veranderd aan het einde van de follow-up periode.

In Hoofdstuk 8 werd nagegaan of de elektrofysiologische veranderingen die veroorzaakt worden door lang bestaand boezemfibrilleren reversibel zijn bij patiënten bij wie de boezemfibrillatie episoden snel beëindigd werden met de Atrioverter. Vier patiënten met herhaalde episoden van boezemfibrilleren (duur 3 tot 21 jaar, gemiddeld $13 \pm 7.6$ jaar) werden gevolgd na implantatie van een Atrioverter. Het toestel analyseert ellk uur het hartritme en slaat dit in zijn geheugen op. Voor de implantatie werd de effectieve refractaire periode van de boezem gemeten. Tijdens een gemiddelde follow-up van 14 maanden traden er 52 spontane episoden van boezemfibrilleren op warvan er 39 behandeld en 18 niet behandeld werden mer de Atrioverter. Tijdens de studie werden de 4 patiënten behandeld met anti-aritmica. $\mathrm{Na}$ tenminste 1000 uren (uitersten: 1052 en 2675 uur) sinusritme, ondergingen de 
patiënten een elektrofysiologisch onderzoek. Voor de implantatie van de Atrioverter, werd boezemfibrilleren geinduceerd met 1 boezemextrasystole bij 3 patiènten en werd de ritmestoornis niet geinduceerd bij de vierde patient. Na een lange periode van sinusritme (> 1000 uur) kon boezemfibrilleren geinduceerd worden bij dezelfde 3 pariënten op dezelfde wijze als voor de implantatie. Bij de pariènt bij wie geen boezemfibrilleren werd geinduceerd, waren de waarden van de rechter boezen effectieve refractaire periode, bepaald door middel van de enkelvoudige extrastimulus techniek tijdens 3 stimulatie cyclus lengten $(600,500$ en $430 \mathrm{~ms})$, vergelijkbaar mer die wan voor de implantatie. Deze studie toonde aan dat bij de onderzochte patiènten met lang bestaande, herhaalde episoden van boezemfibrilleren, de "normale" boezem elektrofysiologie niet kon worden hersteld.

In Hoofdstuk 9 wordt de effectieve en veilige behandeling met de Atrioverter besproken, die gebruikt wordt bij een patient met boezem ritmestoornissen weroorzaakr door een ernstige congenitale hartaandoening.

In Hoofdstuk 10 werd het gebruik van sedatie tijdens cardioversie met de Atrioverter bij 12 patiënten onderzocht. Tijdens de behandeling van boezemfibrillatie episoden in het ziekenhuis, werd intraveneuze sedarie enkel toegediend op verzoek van de patiënt. De effectiviteit, het aantal toegediende schokken en de noodzak tot sedatie werden geanalyseerd. In totaal werden 393 schokken (1.8 \pm 1.6 schokken/boezemfibrillatie episode) toegediend voor de behandeling van 213 boezemfibrillatie episoden; 85 van de 213 episoden $(40 \%)$ werden buiten her ziekenhuis behandeld. In 195 episoden (92\%) werd sinusritme hersteld. Vijf patiênten vroegen nooit om sedatie. Voor ambulant behandelde boezemfibrillatic episoden was geen sedatie nodig. Voor de behandeling van 26 van de 213 episoden (12\%) werden, na toediening van sedatie, 75 schokken roegediend. Het verzoek om sedatie was afhankelijk van het aantal schokken dat toegediend werd voor de behandeling van een boezemfibrillarie episode $(2.9 \pm 2.7$ schokken woor de behandeling van episoden met sedarie vs $1.7 \pm 1.3$ schokken voor de behandeling wan episoden zonder sedarie; $\mathrm{p}<0.001$ ). De additionele schokken werden toegediend voor de behandeling van onmiddellijke reinitiatie van boezemfibrilleren na een geslaagde defibrillatie ( 14 episoden) of na een inirieel gefaalde cardioversie (4 episode). Tijdens de behandeling van 8 episoden werd vór de toediening van de eerste schok om sedatie verzocht. De studie suggereert dat bij een geselecteerde groep patiënten boezemfibrilleren behandeld kan worden met de Atrioverter zonder gebruik te maken van sedarie. Een succesvolle ambulante behandeling wan een boexemfibrillatie episode vereist geen sedarie, als de Atrioverter geprogrammeerd wordt op her toedienen van maximaal 2 schokken. Wanneer er meerdere schokken nodig waren om een boezemfibrillatie episode te behandelen, nam het werzoek om sedatie toe, eveneens bij parięruten die aanvankelijk geen sedatie nodig hadden.

In Hoofdstuk 11 wordt de eerste evvaring met het ambulant gebruik van de Atrioverter beschreven. Aan deze studie namen 105 patienten deel. Tenminste 3 maanden na implantatie, werd het roestel in een "monitoring mode" geprogram- 
meerd en werden de boezemfibrillatie episoden gedefibrilleerd in aanwezigheid van een arts. Daarna had de arts de mogelijkheid her toestel zo te programmeren dat de patient zelf het toestel kon activeren of dat het toestel auromatisch functioneerde. Aan de patiènten werd gevraagd een vragenlijst in te vullen over de ernst van hun symptomen en een visueel analoge schal woor de analyse van het ongemak en de tevrederheid van de behandeling na elke episode van boezemfibrilleren. Van de 105 patiënten, haddèn 48 patiénten een follow-up van tenminste 3 maanden en deze patiënten voldeden aan de criteria voor ambulante behandeling met de Atrioverter. De 48 pariënten werden behandeld voor 275 boezemfibrillatie episoden. De effectiviteit van de behandeling was $90 \%$ en er werden gemiddeld $1.6 \pm 1.2$ schokken per episode toegediend (mediaan 1). Voor de geslaagde defibrillaties was de VAS score voor ongemak $5.2 \pm 2.4$ en deze score bedroeg $4.2 \pm 2.2$ voor de niet-geslaagde defibrillaties ( $p>0.05$ ). Patinten bij wie de Atrioverter niet had gezorgd voor een succesvolle behandeling van een episode wan boezemfibrilleren waren duidelijk minder tevreden dan zij bij wie de therapie wel succeswol was (VAS score $3.4 \pm 3.3$ vs $8.7 \pm$ $1.3, p<0.05)$. Ook in deze studie induceerden de toegediende schokken geen kamer ritmestoornissen. De eerste ervaring met het ambulant gebruik van de Atrioverter toont aan dat de meeste episoden van boezemfibrilleren veilig en succesvol kunnen worden behandeld. De pariënten zijn tevreden en hebben slechts weinig hinder van een schok indien de Atrioverter het boezemfibrilleren converteert naar sinusritme. 


\section{Dankwoord}

Mijn oprechte erkentelijkheid gaat in de eerste plaats uir naar Prof. dr. H.J.J. Wellens, mijn mentor, die mij de mogelijkheid gaf een grote wens te verwezenlijken. Vooral zijn geloof in een persoon leidt ertoe dat iemand tracht het beste van zichzelf te geven. Zijn grondige kennis en vooruitziende blik in de Cardiologie vormden de basis voor dit proefschrift.

Zonder de hulp en her enthousiasme van Luz-Maria Rodriguez, mijn co-promotor, had ik dit werk onmogelijk kunnen verrichten. Niet alleen heb ik veel geleerd van haar technische vaardigheden en electrophysiologische kennis maar ik heb ook grote bewondering voor haar niet aflatend doorzertingsvermogen wat haar tot een zeer bijzonder persoon maakt.

I would like to express my gratitude to Gregory Ayers who, wherever he was, always found the time to help me in working out study protocols, and articles. His energy has no limits.

De samenwerking met Hendrik Lambert was bijzonder aangenaam. De begeestering voor zijn werk siert hem.

Ilk zou graag Joep Smeets danken voor her onderwijs wat ik van hem heb ontvangen en de samenwerking rond de boezemdefibrillator implantaries.

Graag wil ik vooraanstaande wetenschappers als Professor RS Reneman, Professor MA Allessie, Professor HJGM Crijns, en Professor HAJ Struijker Boudier bedanken voor hun kritische analyse van het proefschrift.

J'exprime ma profonde gratitude au Professeur S. Lévy pour avoir evalué et approuvé cetre thèse.

De steeds gedetailleerd uirgevoerde echocardiografische onderzoeken verricht door collegae Emile Cheriex, Jan-Melle van Dantzig en Jan Heijmeriks speelden een belangrijke rol in de torstandkoming van dit proefschrift.

De overige collegae van de afdeling Cardiologie hebben allen gedurende deze periode bijgedragen tot het voltooien van dit proefschrift.

Veel dank gaat uit naar Paul Barenbrug voor zijn hulp tijdens de implantatie van de boezemdefibrillator.

Tevens wil ik graag de afdeling Anesthesie bedanken voor de mogelijkheid de boezemdefibrillator te kunnen implanteren in de "EFO" kamer.

Mijn dank aan de verpleegkundigen wan de "EFO" kamer voor de talrijke overuren. Eric Hahnraths wil ik in het bijzonder bedanken voor de begeleiding van de patiënten bij wie een boezemdefibrillator werd geimplanteerd.

Tevens zou ik graag de verpleegkundigen van de afdelingen B3 en C3 willen bedanken voor de uitstekende opvang en zorg van de pariënten met de boezemdefibrillator. 
$144 \mid$ Dankwoord

Jean Partouns, ik zal niet snel jouw steeds bereidwillige hulp vergeten.

Mijn beide paranimfen, Chris de Zwaan en Gregory Ayers ben ik erkentelijk voor hun hulp in de slotfase van dit proefschrift. 


\section{Curriculum Vitae}

Carl Clement Marie-Louise Maurice Timmermans

22 september 1957 Geboren in Hasselt, België.

$1972-1978$

Grieks-Latijnse Humaniora, Rorselaar

$1978-1980$

Kandidaat in de geneeskundige werenschappen. Vrije Universiteit Brussel.

$1981-1984$

Doctor in de Genees-, Heel- en Verloskunde. Katholieke Universiteit Leuven.

1984-1990 Geneesheer-specialist in Inwendige Geneeskunde. Katholieke Universiteit Leuven.

1990-1995 Staflid verbonden aan de dienst Cardiologie, Universitaire Ziekenhuizen Katholieke Universiteit Leuven.

$1995-1996$ Fellowship Klinische Electrophysiologie. Academisch Ziekenhuis Maastricht

1996 Geneesheer-specialist in Cardiologie.

1997 Geregistreerd als Cardioloog in Nederland 1996-2000 Staflid verbonden aan de vakgroep Cardiologie, Academisch ziekenhuis Maastricht. 



\section{Publications}

\section{ARTICLES IN JOURNALS}

1. Timmermans $\mathrm{C}$, Vrolix M, Vanhaede I, Srammen F, Piessens J, Vercammen E, De Geest H: Ridogrel in the setring of percurancous transhuminal coronary angioplasty. Ans I Cardiot $1991,68: 463-466$.

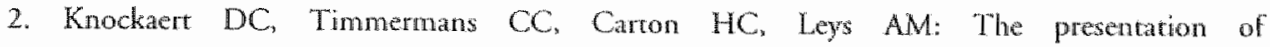
Vogt-Koyanagi-Harada syndrome in internal medicine. Eur J Inrern Med 1992,3:153-156.

3. Timmermans C, Denef B, Herregods MC, Vandeplas A, De Geest $\mathrm{H}$ : Het belang wan transoesofagale echocardiografie in de klinische cardiologie. Tijolschr Giencesk 1992,48: $1635-1640$

4. Herregods MC, Anastassiou I, De Man F, Timmermans C, Vandeplas A, Van De Werf F. De Geest $\mathrm{H}$ : Mogelijkheden en beperkingen van transoesofageale echocardiografie. Tijdschn Geneesk $1993,49: 739-743$.

5. Timmermans C, Ector $\mathrm{H}$ : Prognostische betekenis van late potentialen na myocardinfarct. Tijdschr Geneesk 1993,49:1253-1257.

6. Herregods MC, Timmermans C, Frans E, Decramer M, Daenen W, De Geest H: Diagnosric walue of transesophageal echocardiography in platypnea. J Am Soc Echocardiogr 1993,6:624-627.

7. Timmermans $C$, Frans $\mathbb{E}$, Herregods $C$, Decramer $M$, Daenen W, De Geest H: Platypnea-orthodeoxia syndrome: A repart of two cases. Acra Cardiologica 1993, XLVIII:583-590.

8. Timmermans C, Ector H. Haisty KW, Hammill SC, Kienzle MG, Orawa $Y$, Reddy S, Underwood DA: Genderspecific differences in signal-averaged ECG parameters of cardiac nomals using Frank lead system and Fourrier transform filter: A multicenter study. Pacing Clin Electrophysiol 1994,17.303-311.

9. Oreto G, Smeers JLRM, Rodriguez LM, Timmermans C, Constantinou L, De Sousa M, Vainer I, Merzer J. Wellens HJ]: $P$ wave detection during supraventricular tachycardia. Mew Trends Arrhythmias 1993,9/4:1117-1124.

10. Oreto G, Smeets JLRM, Rodriguez LM. Timmermans C, Wellens HJ]: Supernomal conductions in the left bundle branch. J Cardiovase Electrophysiol 1994,5:345-349.

11. Frans E, Timmermans C, Herregods MC. De Geest H, Daenen W, Delanote M, Decramer M: Plarypnoea syndrome caused by atrial septal aneurysm. Eur Respir I 1994,7:2082-2084.

12. Timmermans C, Sneets JLRM, Rodriguez LM, Oreto G, Medina E, Notheis W, Vrouchas G. Weide $A$, Wellens HJ]: Recurrence rate after accessory pathway ablation. $\mathrm{Br}$ Hean I $1994.72: 571-574$

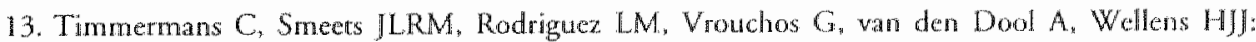
Aborred sudden dearh in the Wolff-Parkinson-White syndrome. Am I Cardiol 1995.76:492-494. 
14. Rodrigue LM, Stemick EB, Smeers, JLRM, Timmermans C, den Dulk K, Orero G, Wellens HJ]: Induction of ventrictalar fibrillation predicts sudden death in patients treated with amiodarone because of ventricular tachyarrhythmias after a myocardial infarcrion. Heart 1996,75:23-28.

15. Morrelmans L, Vanhecke W, Mertens D, Ecror H, Timmermans C, De Roo M, De Geest H, Van de Werf F: Assessment of the oprimal arrioventricular delay in dual chamber-paced patients by a portable scintillation probe (VEST). I Nud Cardiol 1996,3-321-326.

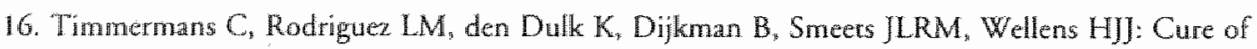
incessant pacemaker circus movement tachycardia by radiofrequency catheter ablation. I Cardiovasc Electrophysial 1996,7:862-866.

17. Oreto G, Smeets JLRM, Radriguez LM, Timmermans $C$, Wellens HJ]: Wide complex rachycardia wirh $A V$ dissociation and QRS morphology identical ro that of sinus thythm: $A$ manifestation of bundle branch re-entry. Heart 1996,76:541-547.

18. Rodriguex LM, Smeets JLRM, Timmermans $C_{s}$ Trappe HJ, Wellens HJ]: Radiofrequency catheter ablation of idiopathic ventricular tachycardia originating in the anterior fascicle of the left bundle branch. J Cardiowasc Electrophysiol 1996,7:1211-1216.

19. Rodriguez LM, Smeets JLRM, Timmermans C, Wellens HJ]: Predicrors for successful ablation of right and left sided idioparthic wentricular tachycardia. Am J Cardiol 1997,79-309-314.

20. Timmermans $\mathrm{C}$, Rodriguez LM, Smeets JLRM Cycle length and QRS alternation during a narrow QRS tachycardia. J Cardiovasc Electrophysiol 1997,8:963-964.

21. Rodriguez LM, Smeets JLRM, Timmermans C, Blommaert D, van Dantzig JM, de Muinck E, Wellens HJ]: Radiofrequency catheter ablation of sustained monomorphic ventricular tachycardia in hypertrophic cardiomyopathy. J Cardiovasc Electrophysiol. 1997,8:803-806.

22. Smeets JLRM, Rodriguex LM. Timmermans C, Wellens HIJ: Radiofrequency catherer ablation of idiopathic wentricular tachycardias in children. Pacing Clin Electrophysiol 1997,20(No 8,part IJ), 2068-2071.

23. Wellens HJI, Rodriguez LM, Timmermans C, Smeets JLRM: The asymptomatic patient with the Wolff-Parkinson-White elecrocardiogram. Pacing Clin Electrophysiol 1997, 20 (No 8,part II).2082-2086.

24. Wellens HJW, Doevendans P, Smeets JLRM, Rodriguez LM, Den Dulk C, Timmermans C, and Vos M: Arrhythmia risk: Elecrrophysiological studies and monophasic action potentials. Pacing Clin Electrophysiol 1997, 20 (No 10,parr II),2560-2565.

25. Harzinikolaou $H$, Rodriguer LM, Sneers JLRM, "Timmermans $C$, Vrothos G. Grecas $G$, wellens HIJ: Isoprenaline and inducibility of atrioventricular modal re-entrant tachycardia. Heart $1998,79: 165-168$.

26. Thimmemans $\mathrm{C}$, Rodriguez LM. Smeets JLRM, Wellens HJJ: Immediate reinitiation of atrial fibrillation following internal arrial defibrillation. J Cardiowasc Electrophysiol 1998,9:122-128.

27. Timmermans C, Rodriguea L.M, Ayers GM, Lambert H, Smeets JLRM, Wellens HJJ.: Effect of dectrode length on arrial defibrillation thresholds. J Cardiowasc Electrophysiol 1998,9:582-587.

28. Smeets JLRM, Ben-Haim BA, Rodriguez LM, Timmermans C, Wellens HJ]: New method for nonfluoroscopic endocardial mapping in humans. Accuracy assessment and first dinical results. Circulation $1998,97: 2426-2432$. 
29. Wellens HJj, Lau CP, Lüderitz B, Akhtar M, Waldo AL, Camm AJ, Timmermans $C$, Tse HH, Jung W, Jordaens L. Ayers $G$, for the Merrix Investigators: The Atrioverter, an implantable device for the treament of arral fibrillation. Circulation 1998,98:1651-1656.

30. Rodriguez LM, Leunissen J, Hoekstra A, Korteling BJ, Smeets JLRM, Timmermans C, Vos M. Daemen M, Wellens HJJ: Transwenous cold mapping and cryoablation of the AV node in dogs: observations of chronic lesions and comparison ro those obtained using radiofrequency ablation. I Cardiovasc Electrophysiol 1998,9:1055-1061.

31. Rodriguez LM, Timmermans C, Restrepo C, Smeets I, Wellens HJJ: Ablacion por radiofrecuencia de raquicardias ventriculares idioparicas en ninos $Y$ adolescentes. Edicion Lationa de Electrocatdiologia 1998,4:76-85.

32. Nabar A, Rodriguez LM, "Timmermans C, Smeets JLRM, Wellens HJ).: Radiofrequency ablation of "Class IC atrial thutrer" in patients with resistant atrial fibrillation. Am J Cardiol 1999, 83:785-787.

33. Nabar A, Rodriguez LM, Timmernans C, van den Dool A, Smeers JLRM, Wellens HJ]: Effec of right atrial isthmus ablation on the occurrence of arrial fibrillation: Obserwations in four patient groups having type I atrial flutrer with or without associated atrial fibrillation. Circulation 1999,99:1446-1451.

34. Timmermans C, Rodriguez LM, Ayers GM, Lambert H, Smeets JLRM, Vlaeyen JWS, Albert A* Wellens HJ]: Effect of butorphanol tartrate on shock-related discomfort durung internal atrial deforillation. Circulation 1999, 99:1837-1842.

35. Nabar A, Radriguez LM, Timmermans C, Smeers JLRM, Wellens HJ]: Isoproterenol to evaluate resumption of conduction after right atrial isthmus ablation in type $\mathrm{I}$ atrial flutter. Circulation $1999,99: 3286-3291$.

36. Rodriguez LM, Timmernans C, Wellens HJI: Are electrophysiological changes induced by longer lasting atrial fibrillation reversible? Observations using the atrial defibrillator. Circulation 1999, 100:113-116.

37. Timmermans $C$, Nabar A, Rodriguez LM. Ayers G, Wellens HJ]: Use of sedation during cardioversion with the implantable atrial defbrillator. Circulation 1999, 100:1499-1501.

38. Timmermans C, Rodriguez LM, Ayers GM, Siu A, Smeers JLRM, Barenbrug P, Wellens HJ: Design and preliminary data of the Metrix ${ }^{\text {TM }}$ Atrioverter expanded indication triat. I Intervent Electrophysiol 2000 , in press.

39. Timmermans C, Rodriguez LM, Reuter D, Stappers J, Barenbrug P, Chericx EC, Wellens HI: Management of arrial arrhythmias secondary to severe congenital heart discase with the Arriowerter. Pacing Clin Electrophysiol 2000, in press.

40. Rodriguez, LM, Nabar A, Timmermans $\mathrm{C}$, Wellens HJl: Should we prefer a long rip electrode to perform radiofrequency ablation of rype I atrial flutter? Results of a prospective randomized study comparing a $8 \mathrm{~mm}$ splir-rip versus a 4 tim tip ablation catheter. Am J Cardiol 2000 , in press.

41. Timmermans $C_{8}$ Lêvy $S$, Ayers GM, Jung W, Jordaens L. Rosenquist M, Thibault B. Camm J. Rodriguez LM, Wellens HJ]: Spontaneous episodes of atrial fibrillation after implantation of the Metrix TM Atrioverter: observations on treated and nontreated episodes. I Am Coll Cardiol, accepted for publication 
42. Timmermans C, Rodriguex LM, Ayers GM, Masset $\$$, Reuter DG, Wellens HJJ: Decreasing the number of leads required for an implantable atrial defibrillator: use of a new 2 -lead system. Am Hearr J, accepted for publicarion.

43. Nabar A, Radrigute LM, Timmermans C, Katreribeck K, Wellens HJ]: Capture and fusion beats during atrial fibrillation and ventricular achycardia. Heart, accepted for electronic publication.

44. Daoud EG, Timmermans C, Fellows C. Hoyt R, Lemery R, Dawson K, Ayers GM, for the Metrix Investigators: Initial clincal experience with ambularory use of an implantable atrial defibrillator for conversion of atrial fibrillation. Submitted for publication.

\section{CHAPTERS IN BOOKS}

1. Timmermans C. Prognostische betekenis van late potentialen na myocardinfarct. In: Aanwinstem in de inwendige geneeskunde, Ed.: G. Vantrappen. Acco 1992, 89-94.

2. Ector $H_{*}$ Daenen W, Timmermans $C$, Sergeant $P$, De Geesn $H$ : Pacemaker infections and delayed relerrall, In: Progress in Clinical Pacing, Eds.: M. Santini, M. Pistolese, A. Alliegro. Futura media services $1992,389-396$.

3. Timmermans C, Ecror H, Haisty KW, Hammill SC, Kienzle MG, Ozawa Y, Reddy BRS, Underwood D: A multicenter study determining signal-averaged ECG parameters and their retation to gender in normal subjects. In: Proceedings of the "Euro-Pace "93 - 6th European Symposium on Cardiac Pacing”, Eds.: A.E. Aubert, H. Ector, R. Stroobants. Monduzzi Editore $1993,117-119$.

4. Rogers $\mathrm{R}$, Ector $\mathrm{H}_{*}$ Rubens A, Timmermans $\mathrm{C}$, Heidbüchel $\mathrm{H}$, De Geest $\mathrm{H}$ : Classification of death in patients under anti-arrhythmic treatment. In: Cardiac pacing and electrophysiology. A bridge to the 21 sr century, Eds.: A.E. Aubert, H. Ecror, R. Stroobandt. Kluwer Academic Publishers 1994, 41-48.

5. Timmermans $\mathrm{C}$, Ecror $\mathrm{H}$ : Herkenten van ventrikeltachycardie op het routine ECG. In: Aanwinsten in de inwendige genceskunde, Ed.J. Fevery. Acco 1994, 55-63.

6. Wellens HJI, Smeets JL, Rodriguez LM, Timmermans C. Are there still indications for pharmacological tratment in patients with $\mathrm{AV}$ reentry tachycardias with and withour accessory AV pathways?' In: Ten years of radiofrequency cacheter ablation, Eds.: Farre J, Moro C. Armonk, NY: Futura Publishing Company, 1998:43-48.

7. Smees JLRM, Ben-Haim S, Rodriguez LM, Timmermans $C$, Wellens HJJ. Prelimenary experience wich an nonfluoroscopic catheter-based mapping method in parients with a accessory pathway. In, Ten years of radiofrequency catheter ablacion, Eds.: Farre I. Moro C. Armonk, NY: Furura Publishing Company, 1998:193-202.

8. Wellens HJd, Smeets JLRM, Rodriguez LM, Timmermans C. Clinical and electrocardiographic profiles of parients wich ventricular tachycardia. In: Ten years of radiofrequency catheter ablation, Eds.: Farre J, Moro C. Armonk, NY: Futura Publishing Company, 1998:205-217.

9. Rodriguez LM, Smeets ILRM, Timmermans C, Wellens HIJ. Predictors of successful ablation of right-and left-sided idiopathic ventricular tachycardia. In: Ten years of radiofrequency catheter ablation, Eds.: Farre J, Moro C. Armonk, NY: Futura Publishing Company, 1998:231-248. 
11. Timmermans $C$, Rodriguez LM, Smeets JLRM, Wellens HJJ. Human experience with implantable atrial defibrillators. In: Progress in Clinical Pacing, Ed.: Santini M. Futura Media Services 1998. 101-104.

10. Timmermans C, Rodriguez LM, Wellens HJJ. The arrial deflbillator. In: Cardiac Electrophysiology: From Cell to Bedside, 3/e, Eds.: Zipes DP, Jalife J. Philladelphia: WB Saunders Company, 1999, 971-974.

12. Rodriguex LM, Timmermans $C_{\text {; }}$ Wellens HIJ. Radiofrequency catheter ablation of supraventricular and wentricular arrhythymias. In: Cardiovascular Medlicine, 2/e, Eds.: Willerson JT, Cohn IN. New York: Churchill Livingsrone, in press.

13. Timmermans C, Rodriguez LM. Wellens HJj. External and internal cardioversion and defibrillarion. In: Hurst's The Heart, 10/e, Eds.: Fuster V, Alexander W, O'Rourke R, Roberts R, King 5 , Wellens H. New york: McGraw-Hill, in press. 